Aus der Abteilung Allgemeinmedizin

(Prof. Dr. med. M. M. Kochen, MPH, FRCGP)

im Zentrum Innere Medizin

der Medizinischen Fakultät der Universität Göttingen

\title{
Methode und Qualität der Adhärenzmessung in randomisiert kontrollierten Studien
}

\section{Systematic Review}

\author{
INAUGURAL - DISSERTATION \\ zur Erlangung des Doktorgrades \\ der Medizinischen Fakultät \\ der Georg-August-Universität zu Göttingen
}

vorgelegt von

Philipp Schönborn

aus

Olpe

Göttingen 2009 
De k a n:

I. Berichterstatter: Prof. Dr. disc. pol. W. Himmel

II. Berichterstatter/in:

III. Berichterstatter/in:

Tag der mündlichen Prüfung: 


\section{Inhalt}

$1 \quad$ Einleitung

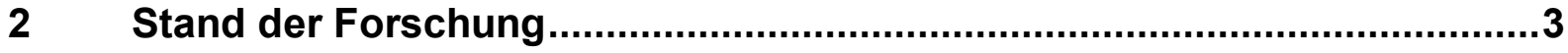

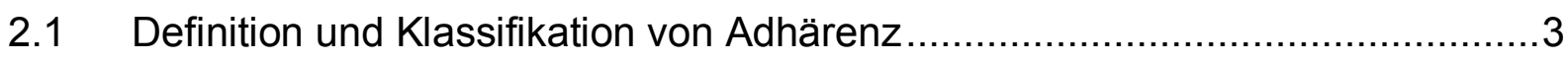

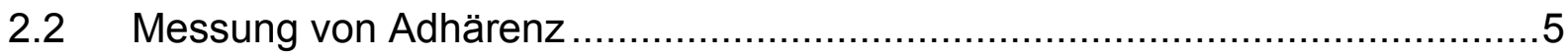

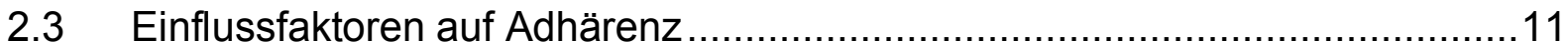

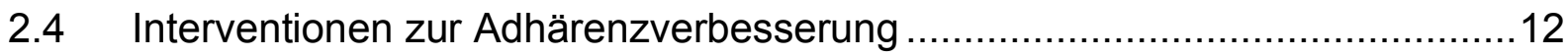

2.5 Bedeutung und Konsequenzen für die Praxis........................................ 13

2.6 Empfehlungen für Adhärenzmessung...................................................14

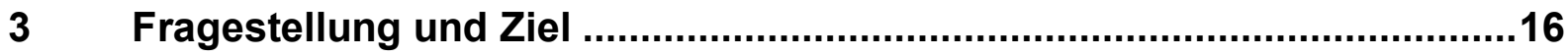

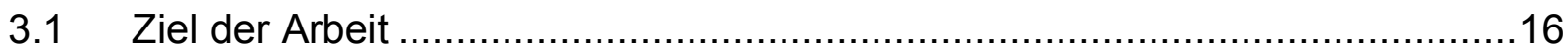

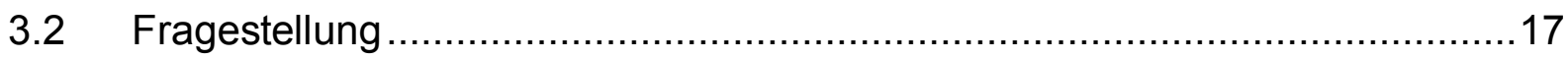

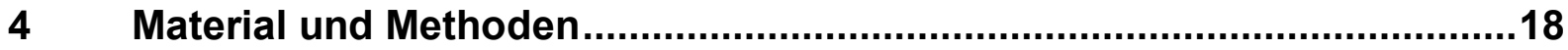

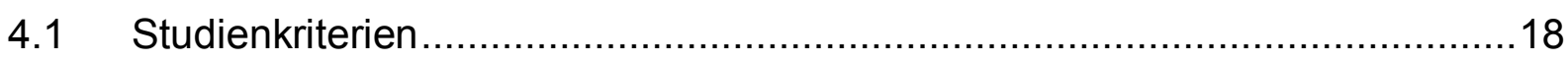

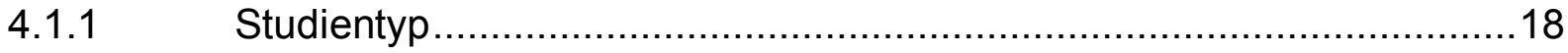

4.1.2 Studienteilnehmer und Einschlussdiagnosen ..................................19

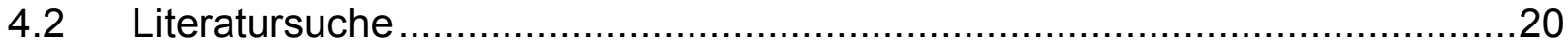

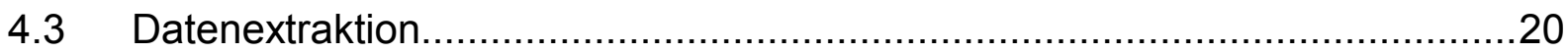

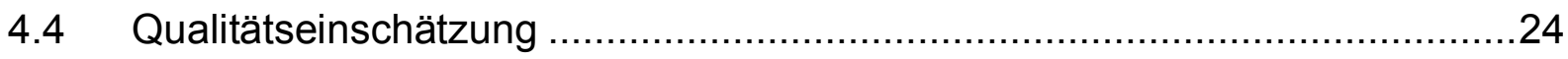

4.4.1 Qualitätsbewertung aller eingeschlossenen Studien .........................24

4.4.2 Qualitätsbewertung der Studien mit mindestens 2 Messmethoden .......24

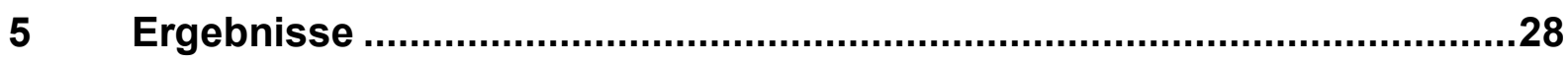

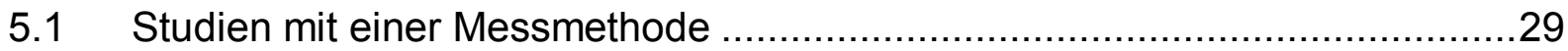

5.2 Studien mit zwei oder mehr Messmethoden ..............................................30

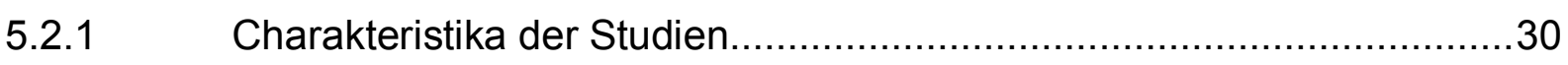

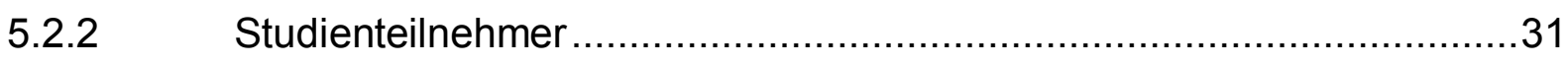

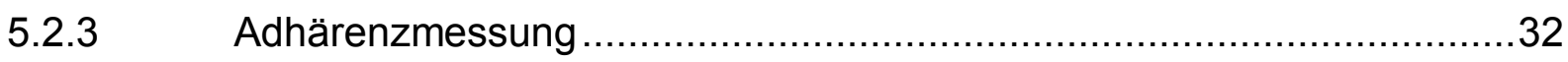

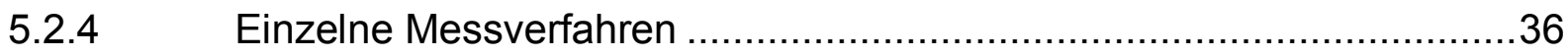

5.2.5 Messmethoden im Studienkontext ............................................42 


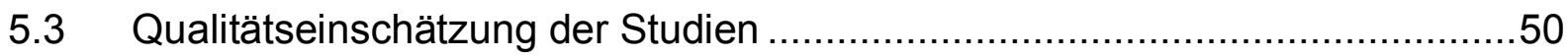

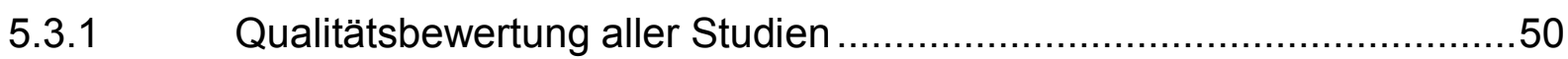

5.3.2 Qualitätsbewertung der Studien mit mindestens 2 Messmethoden .......50

5.3.3 Bewertung von "Self-Reports“ und „Medication Counts/Weighings“ ......52

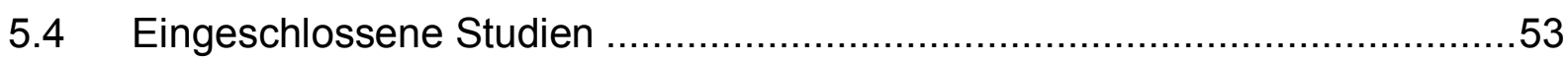

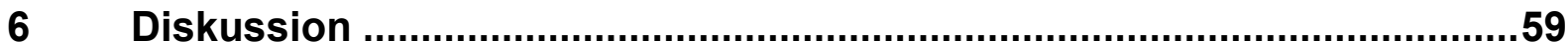

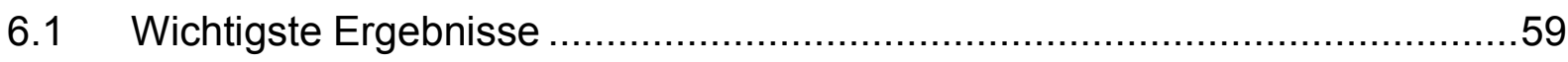

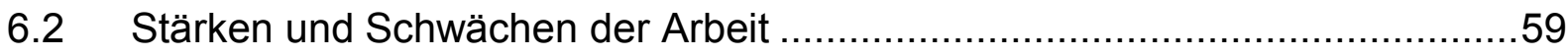

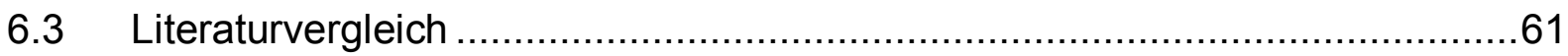

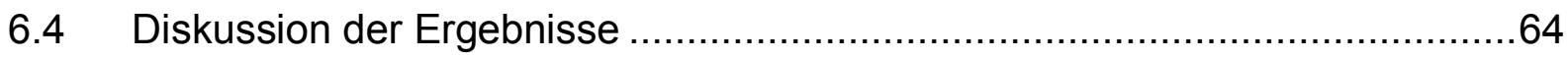

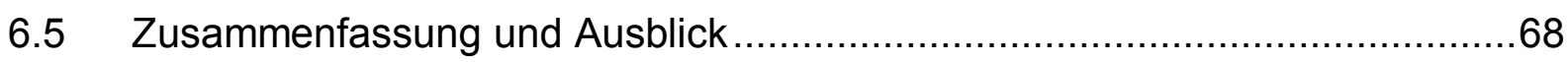

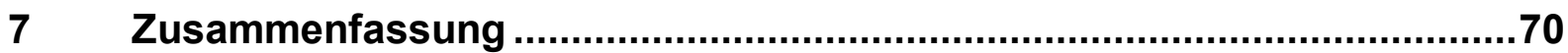

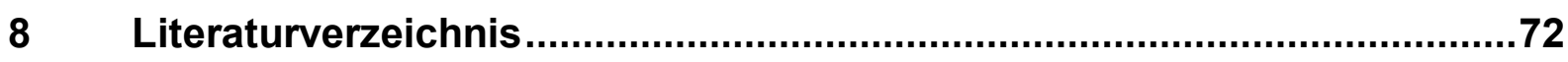

Anmerkung: Aus Gründen der sprachlichen Vereinfachung und zur besseren Lesbarkeit wird im Text auf eine geschlechtsspezifische Differenzierung wie z.B. Arzt/Ärztin verzichtet. Entsprechende Begriffe gelten im Sinne der Gleichbehandlung für beide Geschlechter. 


\section{Einleitung}

Der Begriff Adhärenz beschreibt das Ausmaß der Einhaltung ärztlicher Behandlungsvereinbarungen und spielt gerade in der Behandlung chronischer Erkrankungen eine sehr wichtige Rolle. Obwohl Adhärenz die Wirksamkeit medizinischer Behandlung steigert, Mortalität und Morbidität verzögert bzw. reduziert (Irvine et al. 1999, Simpson et al. 2006), Lebensqualität von Patient (und Arzt) verbessert, Kosten für das Gesundheitssystem senkt und Ressourcen des Gesundheitssystems schont, ist Non-Adhärenz, also die Nicht-Einhaltung von Behandlungsvereinbarungen, weit verbreitet. Schon hier sei einschränkend gesagt, dass Non-Adhärenz ein legitimes Selbstbestimmungsrecht des Patienten ist und in bestimmten Fällen sogar vorteilhaft sein kann (intelligente Non-Adhärenz).

Es ist bekannt, dass Ergebnisse zum Ausmaß von Non-Adhärenz auch von den verwendeten Messmethoden abhängig sind. Die Analyse der Messmethoden stellt also einen elementaren Grundstein für die Adhärenzforschung dar. Mindestens drei Probleme zeigen sich dabei:

1) Es existiert keine allgemeingültige Definition von Adhärenz. Verschiedene Begriffe werden mit z.T. unterschiedlichen Bedeutungen nebeneinander verwendet.

2) Adhärenz ist ein komplexes Modell, das von zahlreichen, zum Teil noch unbekannten Faktoren (und deren Wechselwirkungen) abhängig ist.

3) Da es bisher noch nicht möglich war, einen Goldstandard für die Methode zur Messung von Adhärenz zu definieren, werden verschiedene Methoden mit unterschiedlicher Durchführung verwendet. Dabei ist die Qualität bzw. die Vergleichbarkeit der Messungen nicht ausreichend geklärt.

In den letzten Jahren wurden in diesem Zusammenhang Empfehlungen zur Durchführung der Messung veröffentlicht, wie z.B. die Verwendung von zwei oder mehr Messmethoden für die Bestimmung der Adhärenz eines Patienten oder eines Versuchteilnehmers, die Beschreibung der in der Studie verwendeten Adhärenzdefinition, die Trennung der verschiedenen Adhärenzarten sowie die Messung von objektivierbaren Krankheitsoutcomes. Die Umsetzung dieser Empfehlungen, die eine bessere Qualität und Vergleichbarkeit mit sich bringen soll, ist bisher nicht untersucht. 
Eine systematische Übersicht über die vorhandene Literatur zu erstellen, ist Gegenstand dieser Arbeit - mit dem Ziel, (1) die bisherige Praxis der Adhärenzmessung in Studien darzustellen, (2) die Qualität und Vergleichbarkeit der Studien und Ergebnisse sowie die Umsetzung der bisher gemachten Empfehlungen zur Verbesserung der Adhärenzmessung zu bewerten und (3) die Effektivität und den Einfluss von Adhärenzmessung abzuschätzen. Daraus sollten sich Empfehlungen für die Adhärenzmessung in zukünftigen Studien ergeben. 


\section{Stand der Forschung}

\subsection{Definition und Klassifikation von Adhärenz}

Adhärenz steht für die Einhaltung ärztlicher Behandlungsvereinbarungen. Eine präzise und einheitlich verwendete Definition von Adhärenz existiert allerdings nicht. Dies liegt daran, dass Adhärenz von zahlreichen Faktoren (Patientenmerkmale, Krankheitsmerkmale, Behandlungsmerkmale, Merkmale der Arzt-Patienten-Beziehung) beeinflusst wird und es bisher noch nicht gelungen ist, die komplexen Zusammenhänge zu einem allgemeingültigen Modell zu verdichten. Hinzu kommt, dass viele Fachrichtungen wie Psychologie, Medizin, Krankenpflege und Gesundheitsökonomie auf diesem Gebiet mit verschiedenen Schwerpunkten forschen und dadurch unterschiedliche Modelle von Adhärenz entstanden sind (Kyngas et al. 2000).

Der Begriff Adhärenz wird häufig synonym mit dem Begriff Compliance verwendet. Die beiden Begriffe sind jedoch nicht gleichbedeutend. Die ersten Definitionen von Compliance durch Sackett (1976) bzw. durch Haynes (1978) fokussieren allein das Verhalten des Patienten und inwieweit er sich an die medizinischen Verordnungen hält. Der modernere Begriff Adhärenz betont laut Chesney et al. (2000b) zusätzlich die Verantwortung des Arztes für den Aufbau einer guten Arzt-Patienten-Beziehung und die Beteiligung des Patienten an der therapeutischen Entscheidungsfindung. Während in dem Begriff Compliance der Schwerpunkt auf der medizinischen Seite liegt, wird bei dem Begriff Adhärenz die ethische Bedeutung des freien Willens und der Eigenverantwortlichkeit des Patienten hervorgehoben. Die International Society for Pharmacoeconomics and Outcomes Research (ISPOR) betont zwar die Vorteile des Wortes Adhärenz, spricht sich aber aus Gründen der Popularität für die weitere Verwendung des Begriffes Compliance aus. Es gibt des Weiteren keinen Hinweis dafür, dass Patienten Adhärenz vorziehen bzw. Compliance als abwertend empfinden (Cramer et al. 2008).

Weitere Begriffe im Zusammenhang mit Adhärenz sind Konkordanz und Persistenz. Konkordanz beschreibt die beidseitige Übereinstimmung von Arzt und Patient bei der Therapieentscheidung; der Begriff Persistenz umschreibt, wie lange ein Patient ein Behandlungsregime einhält. Düsing hat 2006 - mit Bezug auf den deutschen 
Sprachraum - eine verfeinerte Nomenklatur angeregt. Er unterteilt die mangelnde Therapietreue, die er auch als Non-Adhärenz bezeichnet, in Non-Akzeptanz, NonPersistenz und Non-Compliance. Wird das verschriebene Medikament nicht in der Apotheke eingelöst, spricht man von Non-Akzeptanz. Das eigenmächtige Einstellen der Medikamenteneinnahme wird als Non-Persistenz bezeichnet. Andere Unregelmäßigkeiten der Medikamenteneinnahme werden als Non-Compliance bezeichnet.

Non-Adhärenz lässt sich nach Wilker (1994) - mit Bezug auf die verschiedenen Ursachen - noch weiter unterteilen in:

- Adaptive Non-Adhärenz: Patient fühlt sich durch die Therapie in seiner Lebensqualität eingeschränkt und passt sie an seine Bedürfnisse an.

- Beanspruchungs-Non-Adhärenz: Der Therapieplan überfordert den Patienten und es kommt dadurch zu Non-Adhärenz.

- Intelligente Non-Adhärenz: Der Patient befolgt eine ärztlichen Empfehlung nicht, weil Nebenwirkungen aufgetreten sind und/oder der gewünschte Erfolg ausgeblieben ist.

Durch die oben aufgeführte Problematik der verschiedenen Ebenen und Definitionen und die fließenden Übergänge der Begrifflichkeiten ist eine präzise Verwendung schwierig und es können leicht Missverständnisse entstehen. So können Begriffe in einem Zusammenhang synonym verwendet werden, im nächsten Zusammenhang jedoch unterschiedliche Bedeutungen haben.

Diese Arbeit wird sich an der Nomenklatur von Düsing (2006) orientieren. Im Zweifelsfall wird der Begriff Adhärenz bzw. Non-Adhärenz verwendet ${ }^{1}$.

In Zusammenhang mit Medikamenteneinnahme kann Adhärenz auch vereinfacht als das Verhältnis von eingenommener zu verordneter Medikamentenmenge definiert

\footnotetext{
${ }^{1}$ Bei Originalzitaten kann es trotzdem zur Verwendung des Begriffs Compliance kommen. Bei nicht weiterer Erklärung ist der Begriff dann mit dem hier in der Arbeit verwendeten Begriff Adhärenz gleichzusetzen.
} 
werden. Aus der entstehenden Prozentzahl schlagen Heuer et al. (1999) folgende Klassifizierung vor:

Adhärenz 80-100 \% = adhärent

Adhärenz 20-80 \% = teilweise adhärent

Adhärenz $<20 \% \quad=\quad$ non-adhärent

Dieses System basiert auf den Erkenntnissen einer Blutdruckstudie (Haynes et al. 1982a). In dieser Studie konnte bei Patienten mit mindestens 80 \% Adhärenz eine Reduktion des vorher erhöhten Blutdrucks festgestellt werden. Die Problematik der sogenannten Hyperadhärenz (eine Adhärenz über 100 \%) wird in dieser Systematik nicht berücksichtigt. Des Weiteren ist dieses Modell nicht allgemeingültig anwendbar, da die Art der Behandlung bzw. der Erkrankung mit berücksichtigt werden muss. So ist z.B. in der HIV-Therapie eine Adhärenz von mehr als $95 \%$ erforderlich, um die Virussuppression aufrechtzuerhalten (Bartlett 2002). D.h. also, dass ein Patient mit einer Adhärenz von 85 \% gar nicht oder nur bedingt von der Behandlung profitiert. Ein Gegenbeispiel zu den hohen Adhärenzanforderungen bei der antiretroviralen Therapie beschreibt Markowitz (1970): Kinder, die nur ein Drittel der verschriebenen Menge eines Antibiotikums zur Prophylaxe des rheumatischen Fiebers eingenommen hatten, waren auch geschützt.

Die Verwendung eines Cut-offs ist also durchaus sinnvoll oder gar notwendig, um Adhärenz auf den für den Patienten sinnvollen Effekt auszurichten (DiMatteo und Haskard 2006). Gerade adhärenzverbessernde Interventionen sollten Cut-Off-Werte verwenden, um den Anteil der Patienten zu ermitteln, die den für eine OutcomeVerbesserung „erforderlichen“ Adhärenzbereich nach der Intervention erreicht haben.

\subsection{Messung von Adhärenz}

Es gibt zahlreiche Methoden, um die Adhärenz bei medikamentöser Therapie zu messen. Dabei werden direkte und indirekte Messverfahren unterschieden. Bei den direkten Messverfahren wird die Einnahme beobachtet oder es kommt zu einer Messung des Wirkstoffes (bzw. eines Metaboliten oder eines Markers) im Plasma oder im Urin. Direkte Messverfahren liefern zwar verlässlichere Ergebnisse als die indirekten 
Methoden, allerdings sie sind auch aufwendiger, kostenintensiver und häufig invasiv. Ihre Verwendung ist somit nicht immer möglich oder fragwürdig.

Indirekte Messverfahren bestehen u.a. aus Patientenbefragungen in Form von Interviews, Fragebögen und Medikamententagebüchern. Die Durchführung dieser Messverfahren ist kostengünstig und einfach. Außerdem bieten diese indirekten Messmethoden einen großen Vorteil, indem sie einen Einblick in den Adhärenzprozess des Patienten ermöglichen und damit zu einem weiteren Verständnis des Adhärenzmodells führen können (Kyngas et al. 2000). Die Ergebnisse sind nicht so genau, da der Patient sich in der Regel gewissenhafter einschätzt, als er es in Wirklichkeit war, so dass es zu einer Überbewertung der Adhärenz kommt (Vermeire et al. 2001). Insgesamt sind die indirekten Messverfahren aufgrund der einfacheren und kostengünstigeren Anwendungsmöglichkeiten weiter verbreitet als die direkten Messverfahren. Die Vor- und Nachteile der einzelnen Methoden sind noch einmal in Tabelle 1 aufgeführt. Des Weiteren werden subjektive und objektive Messmethoden unterschieden. Zur subjektiven Messung gehören z.B. Self-Report und Kollateralreport, während zur objektiven Messung Medication Count/Weighing, Electronic Drug Monitoring ${ }^{2}$ (EDM), Pharmacy Refill Records sowie Laborbestimmungen gezählt werden (Definition sowie Vor- und Nachteile dieses Verfahrens finden sich ebenfalls in Tabelle 1).

Die größte Metaanalyse über Adhärenz (DiMatteo 2004) hat ergeben, dass diese stark von der Art der Messmethode abhängig ist. Es wurde festgestellt, dass Medication Counts/Weighings eine höhere Adhärenz ermitteln als Kollateralreports, SelfReports, Pharmacy/Medical Records und EDM. Die Metaanalyse unterlag aber einigen Einschränkungen: einerseits wurden z.B. psychiatrische Erkrankungen ausgeschlossen, andererseits nicht-medikamentöse Behandlungen eingeschlossen.

\footnotetext{
${ }^{2}$ Unter Electronic Drug Monitoring versteht man die Adhärenzmessung anhand von Mikroprozessoren in speziellen Medikamentbehältnissen (z.B. Pill Boxes, aber auch Asthma-Spacer u.ä.). Die Mikroprozessoren zeichnen „Öffnung des Medikamentbehältnisses“ bzw. „Auslösung des Spacers“ auf. Diese Daten ermöglichen Rückschlüsse auf Frequenz und Entnahmezeit. Es können also - anders als bei den anderen Messmethoden - auch zeitliche Zusammenhänge der Therapietreue festgestellt werden. Gemeinsames Auswerten der Daten durch Arzt und Patient gilt zugleich als adhärenzverbessernde Intervention.
} 
Tabelle 1 Übersicht über die Messverfahren (nach Bartlett 2002, S.4 und Berg und Arnsten 2006, S.80)

\begin{tabular}{|c|c|c|c|}
\hline Methode & Durchführung & Vorteile & Nachteile \\
\hline \multicolumn{4}{|c|}{ Direkte Messverfahren } \\
\hline $\begin{array}{l}\text { Directly Observed } \\
\text { Therapy (DOT) }\end{array}$ & $\begin{array}{l}\text { Fachpersonal beobachtet, wie } \\
\text { Patient Medikation einnimmt }\end{array}$ & $\begin{array}{l}\text { - Theoretisch } 100 \% \text { Mess- } \\
\text { genauigkeit }\end{array}$ & $\begin{array}{l}\text { - Keine reine Messmethode, son- } \\
\text { dern gleichzeitig auch eine Inter- } \\
\text { vention } \\
\text { - Sehr aufwendig; nur in kranken- } \\
\text { hausähnlicher Institution durch- } \\
\text { führbar } \\
\text { - Nur für eingeschränkte Zeit ein- } \\
\text { setzbar }\end{array}$ \\
\hline Laborbestimmung & $\begin{array}{l}\text { Messung des Wirkstoffes, } \\
\text { Metaboliten oder der Marker- } \\
\text { substanz im Plasma bzw. Urin }\end{array}$ & - Objektives Messverfahren & $\begin{array}{l}\text { - Kann nur klären, ob Patient Medi- } \\
\text { kament vor der Messung nimmt } \\
\text { - Nur sinnvoll bei genau bekanntem } \\
\text { pharmakologischen Profil des Me- } \\
\text { dikaments; individuelle Unter- } \\
\text { schiede in der Metabolisierung } \\
\text { - Evtl. invasiv }\end{array}$ \\
\hline
\end{tabular}

\section{Indirekte Messverfahren}

\begin{tabular}{|c|c|c|c|}
\hline $\begin{array}{l}\text { Medication Count/ } \\
\text { Weighing }\end{array}$ & $\begin{array}{l}\text { Inhalt des Arzneimittel- } \\
\text { behältnisses wird überprüft }\end{array}$ & - Sehr genaue Messungen & $\begin{array}{l}\text { - Setzt Kooperation des Patienten } \\
\text { voraus } \\
\text { - Aufwendig }\end{array}$ \\
\hline Self-Report & $\begin{array}{l}\text { Patient beantwortet Interviews } \\
\text { bzw. Fragebögen oder führt } \\
\text { ein Medikamententagebuch }\end{array}$ & $\begin{array}{l}\text { - Ermöglicht Einblick in den } \\
\text { - Adhärenzprozess } \\
\text { - Praktisch und günstig }\end{array}$ & $\begin{array}{l}\text { - Subjektives Verfahren; ungenauer } \\
\text { als die objektiven Messverfahren } \\
\text { - Anfällig für Social Desirability } \\
\text { Bias }^{3} \text { und den Ceiling-Effekt }\end{array}$ \\
\hline $\begin{array}{l}\text { Electronic Drug } \\
\text { Monitoring (EDM) }\end{array}$ & $\begin{array}{l}\text { Spezielle Arzneitmittel- } \\
\text { behältnisse (Mikrochip für } \\
\text { Datum und Uhrzeit der Medi- } \\
\text { kamentenentnahme) }\end{array}$ & $\begin{array}{l}\text { - Sehr genaue Messungen } \\
\text { - Zeitliche Zusammenhänge } \\
\text { können festgestellt werden }\end{array}$ & $\begin{array}{l}\text { - Curiosity opening }{ }^{5} \text { und Pocket } \\
\text { dosing } \\
\text { - Teuer }\end{array}$ \\
\hline Kollateralreport & $\begin{array}{l}\text { Arzt, Pflegepersonal, Apothe- } \\
\text { ker, Angehörige oder Freunde } \\
\text { bewerten Adhärenz des Pa- } \\
\text { tienten }\end{array}$ & $\begin{array}{l}\text { Wird in bestimmten Fällen } \\
\text { als Ersatz für Self-Report } \\
\text { eingesetzt (z.B. bei Kin- } \\
\text { dern, bestimmte psychi- } \\
\text { sche Erkrankungen etc.) }\end{array}$ & $\begin{array}{l}\text { - Subjektives Verfahren; ungenauer } \\
\text { als die objektiven Messungen } \\
\text { - Anfällig für Social Desirability Bias } \\
\text { und Ceiling-Effekt }\end{array}$ \\
\hline $\begin{array}{l}\text { Pharmacy Refill } \\
\text { Records }\end{array}$ & $\begin{array}{l}\text { Apotheken zeichnen auf ob } \\
\text { und in welchen zeitlichen } \\
\text { Abständen Rezepte eingelöst } \\
\text { werden }\end{array}$ & $\begin{array}{l}\text { - Ermöglicht Untersuchung } \\
\text { großer Kollektive } \\
\text { - Sehr resistent gegen Soci- } \\
\text { al desirability bias }\end{array}$ & $\begin{array}{l}\text { - Aus Datenschutzgründen hier } \\
\text { nicht gestattet } \\
\text { - Es ist nicht gesichert, dass der } \\
\text { Patient das abgeholte Medikament } \\
\text { auch einnimmt }\end{array}$ \\
\hline Medical Records & $\begin{array}{l}\text { Es werden medizinische Un- } \\
\text { terlagen zur Adhärenz- } \\
\text { abschätzung verwendet }\end{array}$ & & $\begin{array}{l}\text { - Kaum Literatur auf diesem Gebiet; } \\
\text { inhomogene Anwendung }\end{array}$ \\
\hline
\end{tabular}
abschätzung verwendet

${ }^{3}$ Versuchspersonen beantworten Fragen in Studien nicht nur nach ihrer persönlichen Einstellung, sondern auch nach sozialen Normen, die ihrer Meinung nach erwünscht sind - mit der Folge einer Antwort-Verzerrung.

${ }^{4}$ Ceiling-Effekt: Großteil der Messwerte ist nahe oder am Maximum des Möglichen (geringe Varianz beschränkt die Möglichkeit guter Auswertungen).

${ }^{5}$ Patienten öffnen den Behälter bzw. Iösen das Spray mehrmals kurz hintereinander aus, ohne das Medikament einzunehmen.

${ }^{6}$ Patienten entnehmen bei einer Öffnung mehrere Tabletten/Pillen, um z.B. den Medikamentenbehälter nicht mitnehmen zu müssen. 
Zur Validität der einzelnen Messmethoden gibt es sehr unterschiedliche Aussagen. Nach einem aktuellen Systematic Review (Simoni et al. 2006) gibt es eine hohe Korrelation (84 \%) zwischen HIV-Viruslast und der Adhärenzbestimmung aus SelfReports. Vermeire (2001) und Chesney et al. (2000a) haben dagegen festgestellt, dass der Self-Report zu einer Adhärenzüberwertung führt. Berg und Arnsten (2006) führen diese Ungenauigkeit des Self-Reports v.a auf den Ceiling-Effekt (siehe Fußnote 4, S.7) zurück, der verschiedene Gründe haben kann: Question Misinterpretation, Poor Recall oder aber auch Social Desirability Bias (siehe Fußnote 3, S.7). „Wegen des Ceiling-Effekts, wird der Self-Report häufig als spezifisch, aber nicht sensitiv angesehen, um schlechte Compliance zu erkennen“ (Berg und Arnsten 2006, S.81). Ein weiteres ungelöstes Probleme bei diesem Messverfahren ist die Verwendung unterschiedlicher und zumeist nicht validierter Self-Reports, deren Ergebnisse schlecht übereinstimmen (Gao und Nau 2000, Mannheimer et al. 2006, Walsh et al. 2002, Wiener et al. 2004).

EDM wird v.a. in der HIV-Forschung häufig als Goldstandard in der Adhärenzmessung angesehen, da die Adhärenzergebnisse am besten mit der HIV-Viruslast korrelieren (Arnsten et al. 2001, Liu et al. 2001). Sicherlich konnten durch die Entwicklung dieser Mikroprozessorentechnik neue Einblicke in die Adhärenz gewonnen werden, wie z.B. die Entdeckung der Weißkitteladhärenz ${ }^{7}$. Aber dieses Verfahren hat auch Nachteile. Die Verwendung des EDM ist immer noch sehr teuer und es wird fast immer eine finanzielle Förderung durch einen Sponsor benötigt. Außerdem haben Wendel et al. (2001) festgestellt, dass persönliche Adhärenzstrategien mit dem EDM häufig inkompatibel sind und damit die Adhärenz sogar negativ beeinflussen können. Dies gilt besonders bei komplexen Behandlungsregimes wie bspw. bei HIV. Der spezielle Medikamentenbehälter, der aufgrund der hohen Anzahl verschiedener Medikamente recht groß sein kann, müsste immer dem Therapieplan entsprechend mitgeführt werden. Neben praktischen Problemen könnten dabei gerade HIV-Patienten Angst vor einer Stigmatisierung durch diese Messmethode haben. Des Weiteren sind in der Literatur zwei Phänomene beschrieben, die das Adhärenzergebnis verändern (Samet et al. 2001): Curiosity Opening führt zu Adhärenzüberschätzung, während

\footnotetext{
${ }^{7}$ Patienten mit geringer Adhärenz nehmen ein bis zwei Tage vor Arztbesuch die Medikamente korrekt ein.
} 
Pocket Dosing zu Adhärenzunterschätzung führt. In einer Studie von Bova et al. (2005) berichten 37 \% der Studienteilnehmer von Pocket Dosing und 26 \% von Curiosity Opening. Die Besprechung der EDM-Ergebnisse kann gleichzeitig auch als „adhärenzverbessernde“ Intervention angesehen werden und beeinflusst dann natürlich auch das Adhärenzergebnis.

Bei Medication Counts/Weighings muss man zwischen angekündigten und nicht angekündigten Messungen unterscheiden. Angekündigte Messungen finden in einem geplanten Rahmen statt. Die Medikamente werden im Krankenhaus bzw. zu Hause gezählt oder gewogen. Hierdurch kann es zum so genannten Pill Dumping kommen, bei dem Patienten nicht eingenommene Medikamente vor dem Termin entsorgen. Daher wird davon ausgegangen, dass angekündigte Medication Counts/Weighings Adhärenz überschätzen (Pullar et al. 1989, Rudd et al. 1989). Zudem können weitere methodische Probleme entstehen, wenn bspw. der Patient vergisst, die Medikamente zu dem vereinbarten Termin im Krankenhaus mitzubringen. Diese Art von Messung korreliert moderat mit der Viruslast bei HIV-Erkrankten sowie mit der EDM-Messung. Unangekündigte Messungen hingegen konnten in einigen Studien sogar leicht bessere Vorhersagen für die Viruslast bei HIV-Patienten geben als EDM (Bangsberg et al. 2001, Moss et al. 2004). Gleichzeitig betrachten viele Patienten diese Art von Messung als Misstrauen des Arztes. Da die Arzt-Patienten-Beziehung als sehr wichtig für die Adhärenz angesehen wird, ist die Verwendung dieses Messverfahrens wahrscheinlich eher kontraproduktiv.

Pharmacy Refill Records eignen sich gut, um größere Populationen zu untersuchen und um Zusammenhänge zwischen Adhärenz und verschiedenen Outcomes aufzuzeigen. Berg und Arnsten (2006) führen mehrere Studien an, bei denen Adhärenz gemessen durch Pharmacy Refill Records - signifikant mit Viruslast bei HIVErkrankten korreliert. Die Studien sind auch kaum anfällig für den Social Desirability Bias (siehe Fußnote 3, S. 7). Sie sind jedoch nur in bestimmten Settings und nur in Zusammenarbeit mit bestimmten Organisationen (z.B. den großen amerikanischen „Krankenkassen“ Medicaid oder Veterans Administration) durchführbar. Schwierig bzw. unzuverlässig ist die Methode dann, wenn die Patienten ihre Medikamente auch auf einem anderen Weg erhalten können. Außerdem wird vorausgesetzt, dass der Patient die abgeholten Medikamente auch einnimmt. 
Bei aller Unklarheit über die Validität der einzelnen Messmethoden wird von einigen Messverfahren in der Literatur relativ übereinstimmend abgeraten. So ist die beste Form des Kollateralreports - die Einschätzung der Adhärenz durch den behandelnden Arzt (Vincke und Bolton 2002) - unzureichend. Die Einschätzung der Adhärenz durch die Ärzte ist letztlich nicht besser, als wenn die Ärzte geraten hätten (Fogarty et al. 2002, Gross et al. 2002, Murri et al. 2002). Directly Observed Therapy (DOT) ist mehr eine Intervention als eine Messmethode und ist deswegen nur in bestimmten Settings zu empfehlen, z.B. in Studien, in denen Patienten wirklich alle Medikamente vollständig einnehmen müssen/sollen. Adhärenzmessung anhand von Termineinhaltung ist ebenfalls nicht zu empfehlen. Auch wenn das Versäumen mehrerer Termine in der hausärztlichen Praxis manchmal durchaus Rückschlüsse auf ein evtl. ebenso vorhandenes Adhärenzproblem zulässt, so korrelieren beide Variablen nur schwach miteinander (Hasford et al. 1998).

Krankheitsbezogene Parameter sind ebenso ungeeignet, um Adhärenz zu messen, da auch bei einer vollständigen Befolgung der Therapievereinbarung nicht unbedingt eine Besserung der Krankheit auftreten muss, andererseits jedoch auch bei einer niedrigen Adhärenz eine Besserung der Symptomatik möglich ist. „Das Eintreten einer erwarteten Wirkung ist jedoch nur ein sehr restriktiv zu nutzender Indikator für Compliance, da er unter anderem eine fixe Compliance-Wirkungs-Beziehung voraussetzt, eine Bedingung, die fast nie erfüllt ist" (Hasford et al. 1998, S.35). Auch DiMatteo et al. (2002) raten davon ab, Outcomes zur Abschätzung der Adhärenz zu verwenden, da beide Variablen empirisch und konzeptuell völlig unterschiedlich sind. Trotz dieser Einschränkungen ist im Rahmen von Adhärenzmessung eine Erhebung von Krankheitsoutcomes zu empfehlen. Zum einen besteht zumindest eine gewisse Korrelation zwischen Adhärenz und Krankheitsoutcomes, so dass die Erhebung von Krankheitsoutcomes die Validität eines Ergebnisses festigen und komplimentieren kann (Berg und Arnsten 2006, MacLaughlin et al. 2005). Zum anderen ist Adhärenz immer nur sinnvoll, wenn ein für den Patienten nützlicher Effekt entsteht. Aus diesem Grund ist ein genaues Verständnis des Zusammenhangs von Adhärenz und Outcome notwendig (DiMatteo und Haskard 2006). Beispielhaft hierfür ist eine Studie, in der Patienten nach Einnahme von Blutdruckmedikamenten trotz guter Adhärenz keine Besserung des Blutdrucks hatten (Okuno et al. 2002). Hieraus ergeben sich interessante Fragen z.B. über die Korrektheit der Adhärenzmessung bzw. der Outcome- 
messung sowie die Wirkung des Medikaments; diese Fragen wären ohne Verknüpfung der beiden Ergebnisse nicht entstanden. Objektivierbare Outcomes sind dabei eher mit Adhärenz korreliert als subjektive Outcomes (DiMatteo et al. 2002). Die Mitbestimmung von objektivierbaren Outcomes ermöglicht des Weiteren auch die Untersuchung des Healthy Adherer Effects ${ }^{8}$.

\subsection{Einflussfaktoren auf Adhärenz}

Adhärenz wird durch methoden- und kontextabhängige Faktoren beeinflusst (Haynes et al. 1982b). Wie zuvor bereits mehrfach angedeutet, werden zu den methodenabhängigen Faktoren die Adhärenzdefinition und die verwendetete Messmethode gezählt. Bei den kontextabhängigen Faktoren werden krankheitsbezogene, sozioökonomische, therapiebezogene, systembezogene und patientenbezogene Faktoren unterschieden. Einen Überblick über diese kontextabhängigen Faktoren liefert Sabate (2003) in einem Report der WHO (Abbildung 1).

Positiven Einfluss auf die Adhärenz haben: höhere Bildung, höheres Einkommen, nicht-medikamentöses Behandlungsregime, hoher Leidensdruck, bestimmte Erkrankungen wie HIV, Arthritis oder Krebs (DiMatteo 2004). Auch eine gute Arzt-PatientenBeziehung, in der der Patient an der Therapieplanung beteiligt ist, fördert die Adhärenz. Dagegen zeigt sich eine niedrige Adhärenz bei chronischen Erkrankungen im Allgemeinen sowie Diabetes, Schlafstörung und Lungenerkrankungen im Speziellen (DiMatteo 2004). Eine Studie von Cantrell et al. (2006) zeigt, dass Depression, die häufig in Zusammenhang mit chronischen Erkrankungen auftritt, die Adhärenz weiter negativ beeinflusst (nur $43 \%$ des Patientenkollektivs in dieser Studie waren adhärent). Medikamentöse Behandlungsregime, komplizierte Therapiepläne, hohe $\mathrm{Ne}$ benwirkungen, mehrere Medikamente sowie autoritäre Behandlung des Patienten durch den Arzt verringern die Adhärenz.

\footnotetext{
${ }^{8}$ In verschiedenen Studien wurde beobachtet, dass Patienten, die eine gute Adhärenz hatten, auch bessere Krankheitsoutcomes und eine niedrigere Mortalität hatten, obwohl sie in der Placebogruppe waren (Simpson et al. 2006). Dieses Phänomen wird als Healthy Adherer Effect bezeichnet.
} 
- Stadium der Krankheit

- Grad der (drohenden) Einschränkung bzw. Behinderung

- Effektivität der Behandlungsmöglichkeiten

- Vorhandensein von Komorbiditäten

- Schwere der Symptome

\section{Krankheitsbezogene \\ Merkmale}

\section{Sozioökonomische Merkmale \\ - Alter \\ - Bildungsstand \\ - Persönliche wirtschaftliche \\ Situation \\ - Kultureller Hintergrund}

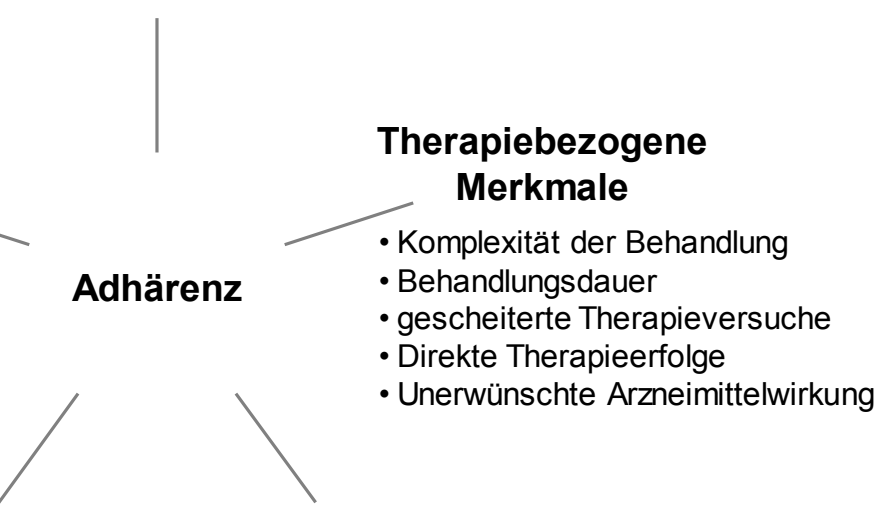

\section{Systembezogene \\ Merkmale}

- Ausbildung und Training

- Arzneimittelverfügbarkeit

- Arzt-Patienten Beziehung

- Systemkapazität

- Art und Dauer der

Untersuchung
Patientenbezogene Merkmale

- Motivation

- Erwartungen

- Wissensstand zur Erkrankung

- Missverständnisse

- Angst vor unerwünschten

Arzneimittelwirkungen

Abbildung 1 Adhärenz beeinflussende Faktoren (nach Sabate 2003, S.27)

\subsection{Interventionen zur Adhärenzverbesserung}

Es existieren zahlreiche Ansätze, Adhärenz zu verbessern (Tabelle 2). Dementsprechend umfangreich ist die Studienlage. Haynes et al. (2005) haben in einer großen systematischen Übersichtsarbeit randomisierte und kontrollierte Studien über adhärenzverbessernde Interventionen ausgewertet. Untersucht wurden Interventionen jeder Art, die zum Ziel hatten, Adhärenz mit verschriebenen und selbst verabreichten Medikamenten zu verbessern. Knapp die Hälfte $(n=31)$ der insgesamt 67 untersuchten Interventionen in 57 Studien erzielte eine statistisch signifikante Verbesserung der Medikamentenadhärenz, allerdings verbesserten nur 22 Interventionen auch die Behandlungsergebnisse. $\mathrm{Zu}$ einem ähnlichen Ergebnis kommt ein HealthTechnology-Assessment-Bericht des Deutschen Instituts für Medizinische Dokumentation und Information (DIMDI), der die Arbeit von Haynes u.a. einschließt (Gorenoi et al. 2007). 
Tabelle 2 Übersicht über Interventionsansätze (modifiz. nach Haynes et al. 2005, S. 5-6)

- Genauere Anleitung und Hilfestellung für Patienten sowie deren Angehörige (mündlich, schriftlich, visuell)

- Beratung und Information bezüglich der Krankheit, der Wichtigkeit von Medikamentenadhärenz und möglichen unerwünschten Medikamentenwirkungen

- Automatisierte telefonische, computer- oder gerätegestützte Medikamentenerinnerung und/oder Beratung

- Persönliche telefonische Beratung und Betreuung

- Familieninterventionen

- Verringerung der Unannehmlichkeiten durch die Behandlung (z.B. Möglichkeit der Behandlung zu Hause oder am Arbeitsplatz)

- Vereinfachte Medikation

- $\quad$ Patientenbeteiligung an der Behandlung und Selbstkontrolle der Ergebnisse (z.B. Blutdruck oder Atmung)

- $\quad$ Erinnerungen (z.B. Anpassung der Behandlung an tägliche Gewohnheiten)

- Spezielle Medikamentenpackungen (Blister)

- Selbstdosierende Medikamentenspender und Medikamententabellen

- Termin- bzw. Rezepterinnerung

- $\quad$ Unterstützung oder Belohnung für verbesserte Medikamentenadhärenz

- Verschiedene Medikamentendarreichungsformen (z.B. Sirup oder Tablette)

- Direkte Beobachtung der Behandlung (DOT) durch Angehörige, Pflegepersonal, Apotheker oder Ärzte

- Übergreifende pharmazeutische Versorgung (z.B. Betreuung durch spezialisierte Pharmazeuten)

- $\quad$ Psychotherapie (z.B. Kognitive Verhaltenstherapie)

Weitere systematische Übersichtsarbeiten bzw. Metaanalysen liefern widersprüchliche Aussagen zur Wirksamkeit von Interventionen. Roter et al. (1998) kommen zu der Schlussfolgerung, dass Interventionen keinen, bzw. wenn, nur einen geringen Effekt haben. Auch nach Takiya et al. (2004) gibt es keine einzelne Intervention, die Adhärenz signifikant verbessert. Eine aktuelle Arbeit zeigt hingegen, dass die Adhärenz bei chronischen Erkrankungen in 20 von 37 Studien durch Adhärenzinterventionen signifikant verbessert werden konnte - jedoch nur in wenigen Fällen mit einem signifikanten Einfluss auf klinische Outcomes (Kripalani et al. 2007).

Auch wenn Non-Adhärenz letztlich Eigenbestimmungsrecht des Patienten ist, so ist es wichtig, die Gründe für dieses Verhalten weiter zu verstehen und - soweit medizinisch und ethisch sinnvoll - zu minimieren, um negative Folgen zu verhindern.

\subsection{Bedeutung und Konsequenzen für die Praxis}

Trotz gewisser Diskrepanzen zwischen Adhärenz und (zu erwartendem) Outcome gilt Adhärenz als sehr wichtig für den Behandlungserfolg verschiedener Erkrankungen. 
Die American Heart Association hat unzuverlässige Medikamenteneinnahme sogar als „Problem Nr. 1“ bei der Behandlung von Herzererkrankungen bezeichnet. Mögliche Folgen von Non-Adhärenz sind (DiMatteo 2004):

- Erhöhte Morbidität und Mortalität des Patienten

- Falsche Forschungsergebnisse und klinische Schlussfolgerungen

- Verschwendung von gesundheitsfördernden Ressourcen sowie hohe Kosten für das Gesundheitssystem (die jährlichen direkten und indirekten Kosten von NonAdhärenz werden auf ca. 10 Milliarden Euro in Deutschland [Heier 2006] bzw. 100 Milliarden Dollar in den USA [Lewis 1997] beziffert).

- Unzufriedenheit, Wut und Frustration von Patient (und Arzt)

- Schlechtere Lebensqualität für Patient (und Arzt).

Die durchschnittliche Non-Adhärenz-Rate beträgt laut DiMatteo (2004) ca. 25 \%, wobei dies stark abhängig von Behandlungsregime, Adhärenzdefinition und verwendeter Messmethode ist. Die Differenz im Behandlungserfolg zwischen niedriger und hoher Adhärenz beträgt 26 \% (DiMatteo et al. 2002). Bei chronischen Erkrankungen, deren Prävalenz vermutlich weiter zunimmt, spielt Adhärenz sogar eine noch größere Rolle.

In ihrer Metaanalyse zeigten Simpson et al. (2006), dass Adhärenz das Mortalitätsrisiko um den Faktor 2 senken kann. Erstaunlicherweise sank die Mortalität auch bei Adhärenz zu Placebos (dies bezeichnet man als Healthy Adherer Effects [siehe Fußnote 8, S.11]). Adhärenz könnte ein Marker für allgemein gesundheitsbewusstes Verhalten sein. Eine neuere Studie findet hierzu jedoch keinen Zusammenhang (Rasmussen et al. 2007).

\subsection{Empfehlungen für Adhärenzmessung}

Es existiert bisher wenig Literatur zu der methodischen Problematik der Adhärenzmessung im Allgemeinen und bei randomisiert kontrollierten Studien im Speziellen (Claxton et al. 2001, Nichol et al. 1999). Die International Society for Pharmacoeconomics and Outcomes Research (ISPOR) hat eine Checkliste veröffentlicht, die die Auswertung retrospektiver Adhärenz- und Persistenzstudien, aber auch die Planung 
solcher Studien erleichtern und verbessern soll (Peterson et al. 2007). Die Arbeit gibt v.a. Empfehlungen zum methodischen Aufbau einer Studie, z.B. welche verschiedenen Aspekte bei der Auswertung berücksichtigt werden sollten (Studiendesign, Datenquellen, Einschluss-/Auschlusskriterien, Studiengruppen, statistische Analysen, Präsentation und Diskussion der Ergebnisse). Es werden auch Empfehlungen zur Bewertung der Adhärenzmessung gegeben. Aufgrund der retrospektiven Ausrichtung werden aber nur bestimmte, grundlegende Aspekte (z.B. Trennung von Persistenz und Adhärenz, Adhärenzdefinition) berücksichtigt. Die verschiedenen Messmethoden werden in dieser Arbeit z.B. nicht behandelt.

Retrospektive Adhärenzstudien machen nur einen Teil aller Adhärenzstudien aus und für prospektive Adhärenzstudien existiert keine vergleichbare Arbeit. Es gibt jedoch verschiedene Empfehlungen zur Adhärenzmessung, um Verzerrungen zu minimieren und die Qualität und Vergleichbarkeit der Messung zu verbessern (Chesney 2006, DiMatteo und Haskard 2006):

- Verwendung mehrerer Messmethoden

- Erfassen von objektivierbaren Krankheitsoutcomes

- Beschreibung der verwendeten Adhärenzdefinition

- Präzise Trennung von Adhärenz und Persistenz

- Beachtung spezifischer Empfehlungen für die einzelnen Messmethoden (z.B. Medication Count/Weighing bei EDM, EDM mit Self-Report koppeln).

Da jede Erkrankung durch krankheitsbezogene Merkmale spezifische Herausforderungen an das Adhärenzverhalten des Patienten stellt, sollten Adhärenzstudien möglichst gezielt ein Krankheitsbild untersuchen.

Die Umsetzung dieser Empfehlungen ist nach unserem Kenntnisstand noch nicht untersucht worden. 


\section{Fragestellung und Ziel}

\subsection{Ziel der Arbeit}

Angesichts der hohen Bedeutung der Adhärenz für Patienten, Ärzte sowie das Gesundheitssystem ist eine genaue Adhärenzmessung sehr wichtig, aber schwierig. Eine wichtige Voraussetzung für eine zuverlässige Messung ist die gleichzeitige Verwendung mehrerer, mindestens zweier Messmethoden mit unterschiedlicher Durchführung. Es ist jedoch weitgehend unbekannt, wie oft dies geschieht und wieweit dies von Studiencharakteristika abhängig ist. Auch die Umsetzung weiterer Empfehlungen (siehe Kapitel 2.2) zur Verbesserung der Adhärenzmessung ist unklar.

In einer systematischen Literaturübersicht wird (1) die bisherige Durchführung der Adhärenzmessung dargestellt, (2) die Qualität und Vergleichbarkeit der Studien und Ergebnisse sowie die Umsetzung der bisher gemachten Empfehlungen zur Verbesserung der Adhärenzmessung bewertet und (3) die Effektivität und der Einfluss von Adhärenzmessung abgeschätzt. Daraus sollten sich Empfehlungen für die Adhärenzmessung in zukünftigen Studien ergeben.

Wir haben unseren Review aus verschiedenen Gründen auf (randomisiert) kontrollierte Studien (RCT) fokussiert. Zum einen werden sie als Goldstandard für die klinische Forschung angesehen (Phillips et al. 2001), da sie (1) durch Randomisation ein Confounding ${ }^{9}$ minimieren, (2) durch Kontroll- und Interventionsgruppe mit gleichen Gegebenheiten für quasi experimentelle Bedingungen sorgen und (3) durch Verblindung Beobachtungsgleichheit erzielen (Willich 2006).

\footnotetext{
${ }^{9}$ Als Confounding bezeichnet man die Verzerrung durch einen Störfaktor (Confounder). Eine scheinbar beobachtete Beziehung zwischen zwei Faktoren ist in Wirklichkeit durch einen dritten („nicht erfassten“) Störfaktor bedingt.
} 


\subsection{Fragestellung}

Im Einzelnen sollte der geplante Review vor allem folgende Fragen beantworten:

- Wie wird Adhärenz in randomisiert kontrollierten Studien gemessen?

- Welche standardisierten Instrumente werden verwendet, um Adhärenz zu messen?

- Wie korreliert die Auswahl von bestimmten Messwerkzeugen mit methodischen Gesichtspunkten der Studien (Land, Patientenzahl, Studiendesign, Intervention vs. Nichtintervention) bzw. Patientencharakteristika (Krankheit, Alter)?

- Werden Empfehlungen der aktuellen Literatur zur Qualitätssicherung der Adhärenzmessung umgesetzt?

- Wie ist die Qualität der Studien im Hinblick auf die Validität der Adhärenz zu bewerten? 


\section{Material und Methoden}

Für das methodische Vorgehen in diesem Systematic Review orientierten wir uns an den Empfehlungen der Cochrane Collaboration (Higgins et al. 2006). In einem apriori-Protokoll wurden Ziele, Studienkriterien und Literatursuche festgehalten. Die Adhärenzdefinition folgt der Nomenklatur von Düsing (2006). Im Zweifelsfall haben wir den Begriff Adhärenz bzw. Non-Adhärenz verwendet (Ausnahme: Originalzitate).

\subsection{Studienkriterien}

\subsubsection{Studientyp}

Adhärenzstudien allgemein lassen sich grob in 3 Gruppen einteilen:

- Studien, die Medikamentenadhärenz in Bezug auf Medikamenteneinführung/wirksamkeit o.ä. untersuchen.

- Studien, die sich mit Adhärenzmessung an sich beschäftigen.

- Studien, die adhärenzverbessernde Interventionen untersuchen.

In der ersten Gruppe spielt die Adhärenzmessung oft eine sekundäre Rolle. Es ist sogar denkbar, dass die Messmethode nach Kriterien ausgesucht wurde, die dem Ziel der Studien entsprechen. Zwei beispielhafte Szenarios hierfür wären:

- Eine Studie ohne Adhärenzmessung wird als nicht vollständig angesehen, deswegen wird ein Self-Report erhoben. Dieser allein ist nicht sehr genau, aber günstig und leicht durchzuführen.

- Eine Pharmafirma will ein neues Medikament testen. Da eine hohe Adhärenz ein wichtiges Argument für die erfolgreiche Vermarktung des Medikamentes ist, wird in der von der Pharmafirma angestellten Studie bewusst ein Self-Report verwendet, da dieser tendenziell die Adhärenz überschätzt.

Solche Studien sind häufig und spiegeln die gängigste Durchführung der „Adhärenzmessung" wider - in scharfem Kontrast zu Studien, die sich gezielt mit Adhärenzmessung an sich beschäftigen. Hierbei werden viele Messmethoden - auch ex- 
perimentelle Verfahren - in verschiedenen Ausführungen verwendet und die Ergebnisse miteinander verglichen, um Adhärenz möglichst genau zu ermitteln. Ein solches aufwendiges Design wäre aber in den meisten anderen klinischen Studien nicht durchführbar.

Studien, die sich mit adhärenzverbessernden Interventionen beschäftigen, sind hingegen für unsere Fragestellung gut geeignet:

- Für die Bewertung der Intervention ist eine genaue Adhärenzmessung wichtig und steht zumeist auch im Mittelpunkt der Studie.

- Allein wegen der hohen Ressourcenanforderungen der Intervention wird auf eine praktische Durchführung der Adhärenzmessung geachtet.

- Experimentelle Messmethoden werden in diesen Studien eher nicht verwendet.

Es wurden also zunächst („Abstract-Screening“) alle kontrollierten Studien eingeschlossen, in denen (1) Medikamentenadhärenz gemessen und (2) eine Intervention zur Adhärenzverbesserung durchgeführt wurde. Nach einer Kurz-Auswertung wurden dann in einem weiteren Schritt (,Volltext-Auswertung“) diejenigen Studien näher ausgewertet, die mindestens zwei unterschiedliche Messmethoden nutzten.

\subsubsection{Studienteilnehmer und Einschlussdiagnosen}

Es wurden Studien unabhängig vom Alter und Geschlecht der Studienteilnehmer eingeschlossen. Studien, in denen man die Studienteilnehmer mit Nahrungsergänzungsmitteln, Kontrazeptiva und Impfmitteln behandelt hatte, wurden aufgrund der z.T. präventiven Situation bzw. der Abwesenheit einer Grunderkrankung ausgeschlossen. Auch Studien mit nicht-pharmakotherapeutischen Behandlungsansätzen, wie z.B. physikalische Übungen, Operationen, Radiotherapien oder Vorsorgeuntersuchungen, wurden ausgeschlossen, ebenso Studien, in denen Kontroll- und Interventionsgruppen mit unterschiedlichen Medikamenten behandelt wurden. 


\subsection{Literatursuche}

Wir haben in den Datenbanken MedLine (1950 bis Januar 2007), Embase (1980 bis Januar 2007), Cochrane Controlled Trials Register (1970 bis 4. Quartal 2006), ClNAHL (1982 bis Dezember 2006) und PsycINFO (1967 bis Januar 2007) mit der in Abbildung 3 abgebildeten Strategie nach publizierten Studien gesucht. Eine Handsuche in den Referenzlisten aller untersuchten Artikel sowie in relevanten Reviews zum Thema Adhärenzmessung aus der Cochrane Library ergänzte die Recherche.

\subsection{Datenextraktion}

Die Daten wurden von zwei unabhängig voneinander arbeitenden Personen (Doktorand und eine medizinische Hilfskraft aus dem 7. medizinischen Semester) extrahiert und in eine standardisierte Form übertragen. Differenzen bei den Auswertungen wurden - ggf. zusätzlich mit weiteren Mitarbeitern der Abteilung Allgemeinmedizin geklärt, bis Einigkeit bestand. Im ersten Auswertungsschritt (Abstract-Screening) wurde die verwendete Messmethode (Abbildung 4) sowie die Grunderkrankung der Studienteilnehmer (Abbildung 5) ausgewertet und kodiert. 


\title{
Suchstrategie für MEDLINE, Suchstrategie für PsycINFO, CINAHL und EMBASE

\author{
Medline In-Process, CCRT (Cochrane \\ Controlled Trial Register) und \\ CL (Cochrane Library)*
}

\author{
1. improv\$ \\ 2. enhanc\$ \\ 3. intervention $\$$ \\ 4. noncomplian\$ \\ 5. non-complian\$ \\ 6. nonadheren\$ \\ 7. non-adheren\$ \\ 8. complier\$ \\ 9. comply \\ 10. complian\$ \\ 11. adhere $\$$ \\ 12. regimen $\$$ \\ 13. drug therapy \\ 14. medicat\$ \\ 15. \#1-3/ OR \\ 16. \#4-11/ OR \\ 17. \#12-14/ OR \\ 18. \#15 ADJ6 \#16 \\ 19. \#17 AND \#18 \\ 20. exp patient compliance/ \\ 21. exp patient dropouts/ \\ 22. exp treatment refusal/ \\ 23. exp patient education/ \\ 24. exp self efficacy/ \\ 25. exp cooperative \\ behaviour/ \\ 26. exp illness behavior/ \\ 27. exp psychotherapy/ \\ 28. exp regimen $\$ /$ \\ 29. \#20-28/ OR \\ 30. \#19 AND \#29
}

1. improv\$

2. enhanc\$

3. intervention $\$$

4. noncomplian $\$$

5. non-complian\$

6. nonadheren $\$$

7. non-adheren\$

8. complier\$

9. comply

10. complian\$

11. adhere $\$$

12. regimen $\$$

13. drug therapy

14. medicat\$

15. \#1-3/ OR

16. \#4-11/ OR

17. $\# 12-14 /$ OR

18. \#15 ADJ6 \#16

19. \#17 AND \#18

\author{
\$ Trunkierung / Wildcard / Platzhalter \\ ADJ6 Suchbegriff wird im Umkreis von 6 Wörtern gesucht \\ exp Es werden alle Studien gesucht, die mit diesen Schlagworten (von den Datenbanken \\ vergeben) verschlüsselt wurden. \\ \# Verweist auf die Schritte der Suchstrategie („\#1-3/ OR“ bedeutet „improv\$ or \\ enhanc\$ or intervention\$“) \\ Die Cochrane Library wurde nur für die Identifizierung relevanter Reviews zum \\ Thema genutzt. Diese wurden dann durch eine „Hand-Search" nach relevanten \\ Studien durchsucht.
}

\section{Abbildung 3 Suchstrategie}




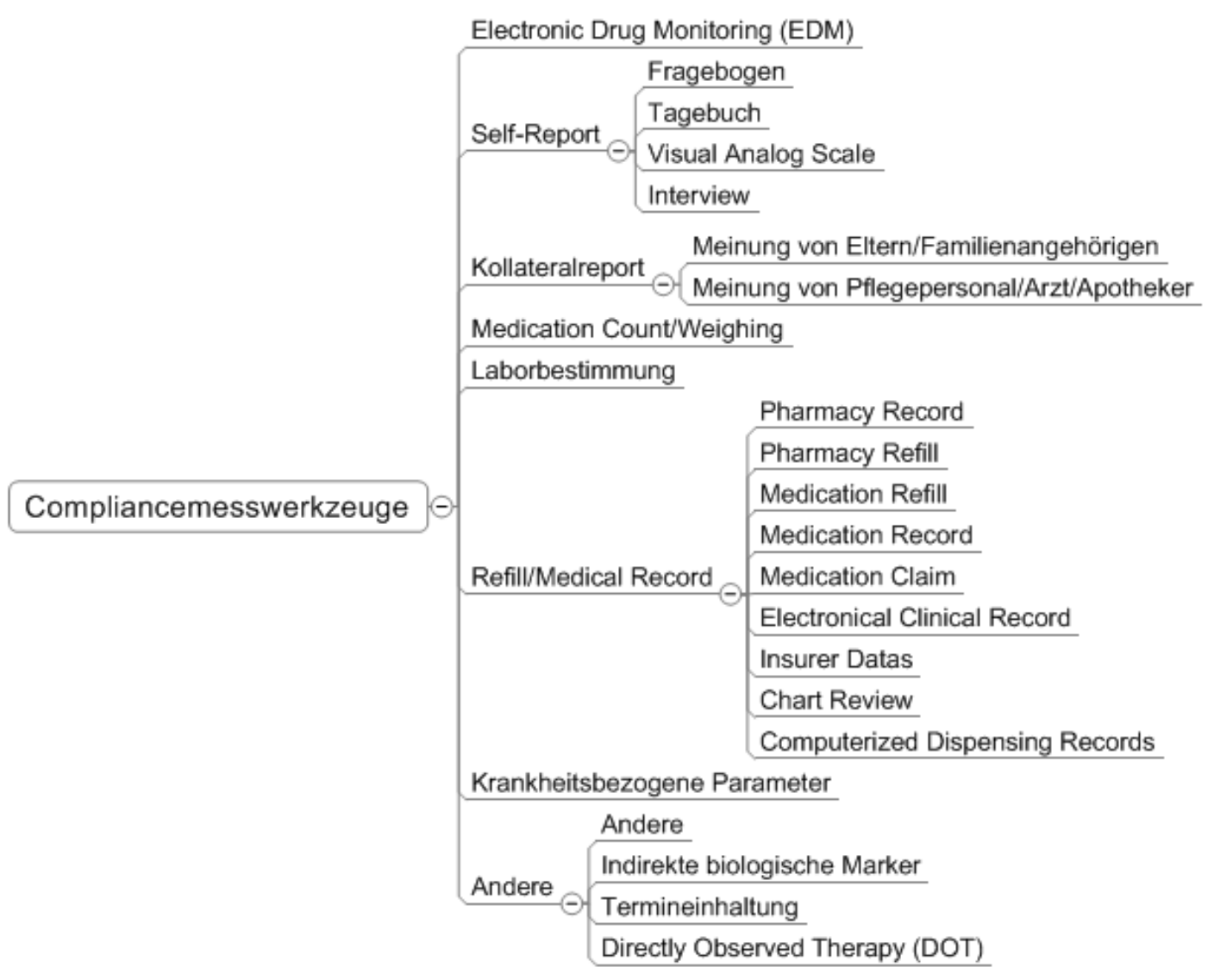

Abbildung 4 Kodierung der Messwerkzeuge

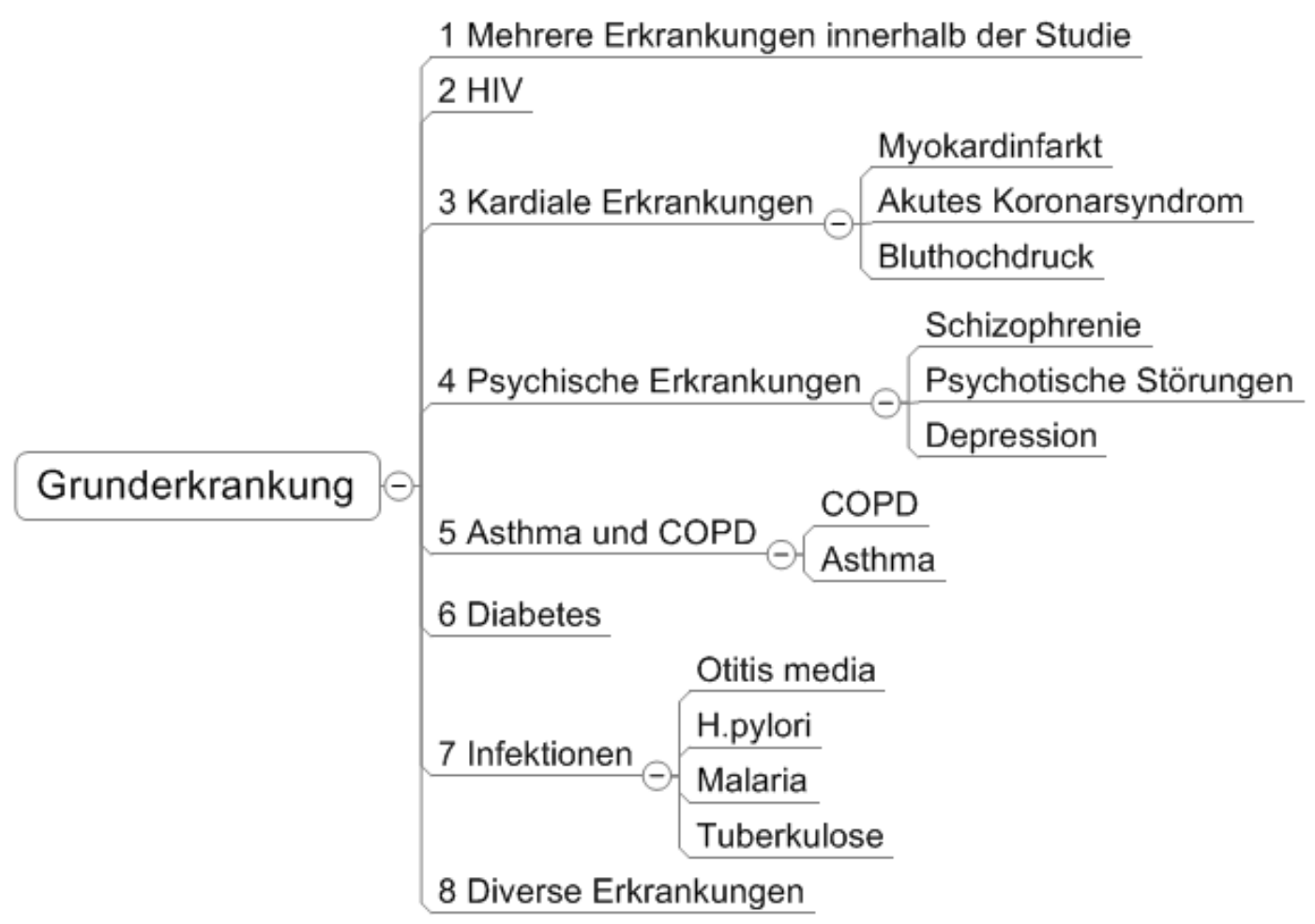

Abbildung 5 Kodierung der Grunderkrankung 
Für die Studien des zweiten Auswertungsschritts (Volltext-Auswertung) wurden neben den verwendeten Messmethoden je nach Möglichkeit noch weitere Outcomes festgehalten und wie folgt verschlüsselt:

- Publikationsdatum

- Randomisation o Ja

$\circ$ Nein

- Studienaufbau

- Parallel

- Crossover

- Faktoriell

o Anderer

- Förderung durch

- Kommerzielle Organisation

- Nicht-kommerzielle Organisation

- Kommerzielle und nicht-kommerzielle Organisation

- Keine Förderung

- Keine Angabe

- Interessenkonflikt $\quad$ Kein Konflikt angegeben

- Konflikt angegeben

- Keine Angabe

- Land

- Nordamerika (USA, Kanada)

- Europa

- Andere

- Studienteilnehmer (Anzahl)

- Durchschnittsalter der Studienteilnehmer

- Geschlechterverteilung

- Studiendauer

- Messmethoden (Anzahl)

- Art der Mitteilung des Adhärenzergebnisses (prozentuale Angabe vs. kategoriale Angabe)

- Erfassung von objektivierbaren Krankheitsoutcomes (z.B. Blutdruck bei Hyptertonie, Viruslast bei HIV)

- Cut-off-Grenzen für Adhärenz bzw. Non-Adhärenz bei qualitativer Adhärenzmessung

- Trennung von Terminadhärenz (=Einhaltung von Terminen; synonym: Termincompliance) und Medikamentenadhärenz

- Aufführung der einzelnen Messmethodenergebnisse

- Angabe von Messzeitpunkt und Messhäufigkeit.

In einem Freitext hielten wir Besonderheiten oder Mängel der Messmethode fest, die nicht kodierbar waren. Von Interesse waren besonders Unterschiede bei der Durchführung der einzelnen Messmethoden (z.B. wurde ein validierter Self-Report verwendet?; welcher?; wenn nicht, welche Fragen wurden beim Self-Report gestellt? war 
der Medication Count/Weighing angekündigt oder unangekündigt?) und Durchführungsbesonderheiten. Ein weiteres Freitextfeld erlaubte die Nennung von weiteren Besonderheiten der Studie (z.B. schlechte Beschreibung der Messmethodik, Durchführungsbesonderheiten der Messmethodik, fehlende Angaben zu Studienteilnehmern, o.ä.).

\subsection{Qualitätseinschätzung}

Ein Verfahren zur Qualitätseinschätzung der Studien wurde nach ausführlicher Literaturrecherche entwickelt. Von entscheidender Bedeutung ist zunächst, dass mehr als eine Messmethode verwendet wird (siehe Kapitel 4.4.1). Soweit dies in einer Studie der Fall war, kamen weitere Kriterien zur Qualitätseinschätzung hinzu (siehe Kapitel 4.4.2).

\subsubsection{Qualitätsbewertung aller eingeschlossenen Studien}

Für den ersten Teil der allgemeinen Adhärenzmessung war die Anzahl der Messmethoden entscheidend. Gemäß der Literatur zur Adhärenzmessung sollen mehrere Messinstrumente verwendet werden (Chesney 2006, DiMatteo und Haskard 2006, Farmer 1999, MacLaughlin et al. 2005). Jedes einzelne Messinstrument hat in verschiedenen Bereichen Defizite (siehe Kapitel 2.2), so dass eine Kombination aus verschiedenen Messinstrumenten diese Defizite zumindest minimiert. Für die Qualität der Adhärenzmessung ist jedoch nicht nur die Anzahl der Messmethoden entscheidend, sondern auch deren Validität. Folgende Messinstrumente wurden anhand der Literatur (siehe Kapitel 2.2) als valide angesehen: Self-Report, EDM, Medication Count/Weighing, Refill/Medical Record und Laborbestimmung. Als nicht valide gelten: Kollateralreport, Adhärenzmessung anhand krankheitsbezogener Parameter, Directly Observed Therapy (DOT) und Termineinhaltungen.

\subsubsection{Qualitätsbewertung der Studien mit mindestens 2 Messmethoden}

Im zweiten Auswertungsschritt wurden Studien mit mehr als einer Messmethode genauer analysiert. Dieses geschah anhand weiterer Qualitätskriterien zur Adhärenzmessung (sowie einer speziellen Qualitätseinschätzung der häufig verwendeten Messmethoden Self-Report und Medication Count/Weighing): 
- Kombination aus Anzahl der Messmethoden mit der Validität der Messmethoden (Studien mit mindestens 2 validierten Messmethoden vs. Studien mit nur einer bzw. keiner validierten Messmethode)

- Messung von objektivierbaren Krankheitsoutcomes (ja/nein)

- Trennung von Termin-/Medikamentenadhärenz (ja/nein)

- Ausreichende Aussagen über formelle Aspekte (Nennung von Messzeitpunkten/Messhäufigkeiten)

- Getrennte Aufführung der Messergebnisse (ja/nein)

- Studie untersucht nur ein Krankheitsbild (ja/nein)

- Cut-off-Definition für Adhärenz (ja/nein).

\section{Scorebildung:}

Aus oben genannten Qualitätskriterien haben wir einen Score gebildet, um einen Überblick über die Qualität der Studien zu ermöglichen. Dabei orientierten wir uns an einer Checkliste zur Beurteilung methodischer Qualität von Gesundheitsinterventionen in randomisierten bzw. nicht randomisierten Studien (Downs und Black 1998). In unserem Review erhielt jedes erfüllte Adhärenzkriterium einen Pluspunkt. Bei NichtVorhandensein gab es - zur Gewichtung der Bedeutung der verschiedenen Qualitätskriterien - 0, -1 oder -2 Punkte (Tabelle 3). Die Anzahl und die Validität der Messmethoden sind bei der Adhärenzmessung besonders wichtig und wurden deswegen bei Nicht-Erfüllung am stärksten negativ gewichtet. Die Definition eines Cutoff-Wertes für Adhärenz bzw. Non-Adhärenz wird zwar gefordert, ihr Fehlen führt jedoch zu keiner Verzerrung. Aus diesem Grund wurde bei Fehlen kein Minuspunkt vergeben. Die Qualität der Adhärenzmessung konnte gut, moderat oder mangelhaft sein:

- Mangelhafte Adhärenzmessung (weniger als 3 Punkte)

- Moderate Adhärenzmessung (4 bis 5 Punkte)

- Gute Adhärenzmessung (6 bis 7 Punkte) 
Zum Erreichen einer guten Adhärenzmessung war also in jedem Fall die Verwendung von mindestens 2 validierten Messmethoden notwendig, sonst hätte es maximal 5 Punkte gegeben. Die Scorebildung führten wieder 2 Personen unabhängig voneinander durch (Doktorand und eine medizinische Hilfskraft aus dem 7. medizinischen Semester); Unstimmigkeiten wurden in Konsens gelöst.

Tabelle 3 Gewichtung der Qualitätskriterien

Kriterium

\begin{tabular}{lcc} 
Qualitätskriterien & erfüllt & nicht erfüllt \\
\hline Mindestens 2 valide Messmethoden & +1 & -2 \\
Messung von objektivierbaren Krankheitsoutcomes & +1 & -1 \\
Trennung von Termin-/Medikamentenadhärenz & +1 & -1 \\
Ausreichende Aussagen über formelle Aspekte & +1 & -1 \\
Getrennte Ergebnisaufführung der einzelnen Messmethoden & +1 & -1 \\
Studie untersucht nur ein Krankheitsbild & +1 & -1 \\
Cut-off Definition für Adhärenz bzw. Non-Adhärenz & +1 & 0 \\
\hline
\end{tabular}

\section{Spezielle Bewertung der Adhärenzmessung anhand zweier Messmethoden}

Im Anschluss an die allgemeine Bewertung der Messmethoden haben wir noch eine spezielle Bewertung der häufig verwendeten Messmethoden Self-Report und Medication Count/Weighing durchgeführt.

Beim Self-Report untersuchten wir, ob eine Studie einen validierten Self-Report ${ }^{10}$ verwendete und ob die Form des Self-Reports (Interview, Fragebogen, Patiententagebuch, etc.) mitgeteilt wurde. Unter den nicht validierten Self-Reports gab es jedoch weitere Unterschiede, so dass noch zusätzliche Kategorien eingeführt wurden:

\footnotetext{
10 Mit validierten Self-Reports sind Self-Reports gemeint, zu denen Validierungsstudien existieren. Dies sagt nichts über die wirkliche Qualität des Self-Reports aus und bedeutet nicht, dass dieser zwangsläufig zu empfehlen ist. Die Validierungsstudie ermöglicht aber, den ermittelten Adhärenzwert einzuschätzen, was bei „unvalidierten“ Self-Reports nicht möglich ist.
} 
- Eindeutige Beschreibung, welche Form des Self-Reports verwendet wurde, Durchführung des Self-Reports beschrieben

- Eindeutige Beschreibung, welche Form des Self-Reports verwendet wurde, Durchführung des Self-Reports nicht beschrieben

- Unklar, welche Form des Self-Reports durchgeführt wurde.

Beim Medication Count/Weighing überprüften wir, ob die Messung angekündigt oder unangekündigt durchgeführt wurde bzw. ob dies überhaupt der Beschreibung zu entnehmen war.

Beide Punkte sind für die Einschätzung der jeweiligen Messmethode von großer Bedeutung. Zum einen wird die Verwendung validierter Self-Reports dringend empfohlen (Paterson und Britten 2005), zum anderen sind die Ergebnisse bei unangekündigtem Medication Count/Weighing anders zu bewerten als die bei einem angekündigten Medication Count/Weighing. 


\section{Ergebnisse}

Die Literaturrecherche lieferte im ersten Anlauf 4.456 Studien. Nach dem kriteriengeleiteten Abstract-Screening wurden 4.008 Studien ausgeschlossen; 448 Studien erfüllten die Einschlusskriterien. Studien mit einer Messmethode $(n=336)$ wurden anhand des Abstracts (und nur bei Bedarf anhand des Volltexts) ausgewertet. Studien mit mehr als einer Messmethode $(n=112)$ wurden im Volltext ausgewertet (Abbildung $5)$.

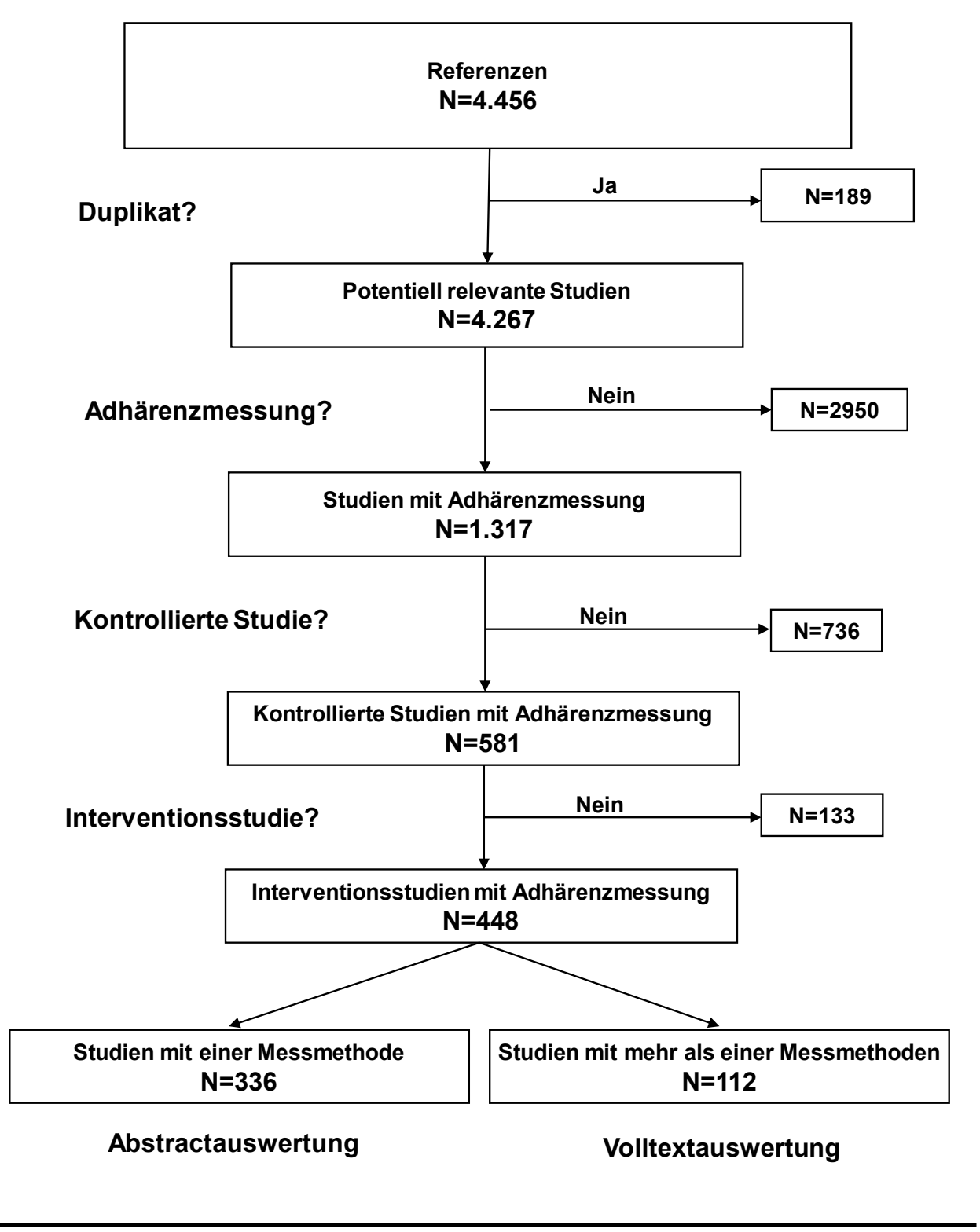

Abbildung 5 Ergebnisse der Literaturrecherche und Artikelauswahl 


\subsection{Studien mit einer Messmethode}

Insgesamt waren 336 Artikel (Interventionsstudien) mit einer Messmethode ausgewählt und genau untersucht worden. Am häufigsten ging es um Herz-KreislaufErkrankungen (Tabelle 4).

Tabelle 4 Grunderkrankung und Instrument

\begin{tabular}{|c|c|c|}
\hline Grunderkrankung $^{\circ}$ & $\mathbf{n}$ & $(\%)$ \\
\hline Mehrere Erkrankungen ${ }^{\wedge}$ & 44 & (13) \\
\hline HIV & 34 & (10) \\
\hline Herz-Kreislauf* & 51 & (15) \\
\hline Psychische Erkrankungen** & 41 & (12) \\
\hline Asthma und COPD & 28 & $(8)$ \\
\hline Diabetes & 19 & (6) \\
\hline Häufige Infektionskrankheiten*** & 29 & (9) \\
\hline Andere & 90 & (27) \\
\hline
\end{tabular}

Instrument $^{\circ}$

$\begin{array}{lrr}\text { Medication Count/Weighing } & 57 & (17) \\ \text { Self-Report } & 151 & (45) \\ \text { EDM } & 47 & (14) \\ \text { Laborbestimmung } & 11 & (3) \\ \text { Kollateralreport } & 15 & (5) \\ \text { Refill/Medical Record } & 34 & (10) \\ \text { Krankheitsbezogene Parameter } & 9 & (3) \\ \text { Andere } & 12 & (4)\end{array}$

$\mathrm{n}=336$

$\wedge$ Hiermit sind Studien gemeint die mehr als eine Erkrankung eingeschlossen hatten

* $\quad$ Bluthochdruck, KHK, Herzinsuffizienz

** Depression, psychotische Störung, Schizophrenie

*** Tuberkulose, akute Otitis media, H. pylori, Malaria

Unabhängig von der Grunderkrankung wurde der Self-Report am häufigsten (im Durchschnitt bei $45 \%$ der Artikel) genutzt (Tabelle 5), etwas seltener bei Artikeln zu Asthma und COPD-Studien (39\%), recht häufig dagegen bei Diabetes-Studien (74 $\%)$.

Studien mit mehreren Erkrankungen bzw. Studien über Herz-KreislaufErkrankungen, Asthma/COPD oder häufige Infektionskrankheiten verwendeten neben dem Self-Report vor allem Medication Counts/Weighings, während Studien über HIV bzw. Studien über diverse Krankheiten als zweithäufigste Messmethode EDM 
verwendeten. Studien über psychische Erkrankungen nutzten neben dem SelfReport am häufigsten Pharmacy/Medical Records und Kollateralreports.

Tabelle 5 Instrumentverteilung bei verschiedenen Grunderkrankungen

\begin{tabular}{|c|c|c|c|c|c|c|c|c|c|c|c|c|c|c|c|c|c|}
\hline & \multirow[b]{2}{*}{$\begin{array}{c}\text { Anzahl } \\
\text { Studien }(n)\end{array}$} & \multicolumn{16}{|c|}{ Typ der Messung; n (\%) } \\
\hline & & \multicolumn{2}{|c|}{ EDM } & \multicolumn{2}{|c|}{ Self-Report } & \multicolumn{2}{|c|}{$\begin{array}{c}\text { Kollateral- } \\
\text { report }\end{array}$} & \multicolumn{2}{|c|}{$\begin{array}{c}\text { Medication } \\
\text { Count/ } \\
\text { Weighing }\end{array}$} & \multicolumn{2}{|c|}{$\begin{array}{c}\text { Labor- } \\
\text { bestimmung }\end{array}$} & \multicolumn{2}{|c|}{$\begin{array}{c}\text { Pharmacy/ } \\
\text { Medical } \\
\text { Record }\end{array}$} & \multicolumn{2}{|c|}{$\begin{array}{c}\text { Krankheits- } \\
\text { bez. Para- } \\
\text { meter }\end{array}$} & \multicolumn{2}{|c|}{ Andere } \\
\hline Mehrere Erkrankungen & 44 & 1 & (2) & 18 & $(41)$ & 2 & (5) & 15 & (34) & 1 & (2) & 7 & (16) & - & - & - & - \\
\hline HIV & 34 & 12 & $(35)$ & 19 & $(56)$ & - & - & - & - & 1 & (3) & 1 & (3) & 1 & $(3)$ & - & - \\
\hline Herz-Kreislauf & 51 & 8 & $(16)$ & 24 & $(47)$ & - & - & 9 & (18) & - & - & 6 & (12) & 3 & $(6)$ & 1 & (2) \\
\hline $\begin{array}{l}\text { Psychische } \\
\text { Erkrankungen }\end{array}$ & 41 & 2 & $(5)$ & 17 & (42) & 71 & (17) & 4 & $(10)$ & - & - & 10 & $(25)$ & - & - & 1 & (2) \\
\hline Asthma und COPD & 28 & 3 & $(11)$ & 11 & (39) & 31 & (11) & 5 & (18) & 1 & (4) & 3 & (11) & 2 & (7) & - & - \\
\hline Diabetes & 19 & 1 & (5) & 14 & (74) & - & - & - & & - & - & 2 & (11) & 1 & (5) & 1 & (5) \\
\hline $\begin{array}{l}\text { Häufige } \\
\text { Infektionskrankheiten }\end{array}$ & 29 & 1 & (3) & 8 & (28) & - & - & 11 & (38) & 4 & (14) & - & - & - & - & 5 & (17) \\
\hline Diverse & 90 & 19 & (21) & 40 & (44) & 3 & (3) & 13 & (14) & 4 & (4) & 5 & (6) & 2 & (2) & 4 & (4) \\
\hline Alle & 336 & 47 & (14) & 151 & (45) & 15 & (5) & 57 & (17) & 11 & (3) & 34 & (10) & 9 & (3) & 12 & (4) \\
\hline
\end{tabular}

\subsection{Studien mit zwei oder mehr Messmethoden}

In 112 Artikeln (ebenfalls Interventionsstudien) gab es mehr als eine Messmethode; diese Artikel wurden im Volltext ausgewertet.

\subsubsection{Charakteristika der Studien}

Fast 90 \% der Studien (97/112) waren randomisiert, zumeist mit paralleler Gruppenbildung. Bei 14 Studien fanden sich keine Aussagen über die Art der Gruppenbildung (Tabelle 6). Fast drei Viertel aller Studien (80/112) waren durch externe Mittel gefördert. Zu den übrigen Studien fanden sich keine Angaben darüber, ob eine Förderung bestand. Von den geförderten Studien wurden $15 \%$ nur industriell und $44 \%$ nur nicht-industriell gefördert (Tabelle 6). In 96 Studien machten Autoren keine Angaben zu einem möglichen Interessenskonflikt; in 3 Studien gaben Autoren einen Interessenkonflikt an. 
Tabelle 6 Methodische und andere Charakteristika der Studien

\begin{tabular}{|c|c|c|}
\hline Methodische Charakteristika ${ }^{\circ}$ & $\mathbf{n}$ & $(\%)$ \\
\hline Randomisation & 97 & (87) \\
\hline \multicolumn{3}{|l|}{ Art der Gruppenbildung } \\
\hline Parallel & 89 & $(80)$ \\
\hline Crossover & 3 & (3) \\
\hline Faktoriell & 6 & (5) \\
\hline Andere & 14 & (13) \\
\hline \multicolumn{3}{|l|}{ Förderung und Finanzierung ${ }^{\circ}$} \\
\hline Gefördert & 80 & $(71)$ \\
\hline Industriell & 17 & (15) \\
\hline Nicht industriell & 49 & (44) \\
\hline Beides & 14 & (13) \\
\hline Keine Angabe & 32 & (29) \\
\hline \multicolumn{3}{|l|}{ Interessenkonflikt ${ }^{\circ}$} \\
\hline Möglicher Konflikt & 3 & (3) \\
\hline Kein Konflikt angegeben & 13 & (12) \\
\hline Keine Angabe & 96 & (86) \\
\hline
\end{tabular}

\subsubsection{Studienteilnehmer}

Tabelle 7 beschreibt die Charakteristika der Studienteilnehmer ( $n=70.167 ; 51 \%$ männlich) aus allen 112 eingeschlossenen Artikeln. Das Durchschnittsalter betrug 42,7 Jahre (SD 18,2; Range 2-87). 15 der 112 Studien befassten sich mit Jugendlichen und Kindern und 17 Studien mit Personen über 60 Jahren.

Die Einschlussdiagnosen in den Studien waren zumeist psychische Erkrankungen (22 \%), gefolgt von Herz-Kreislauf-Erkrankungen und häufigen Infektionskrankheiten (Tabelle 7).

Fast 10 Prozent der Studien (11/112) untersuchten Adhärenzinterventionen bei mehreren, verschiedenen Erkrankungen. Knapp ein Fünftel der Studien war keiner Krankheitsgruppe zuzuordnen.

In Nordamerika wurden mehr als zwei Drittel (68\%) der Studien angefertigt, gefolgt von Europa (19\%). 
Tabelle 7 Soziodemographische Charakteristika der Studien

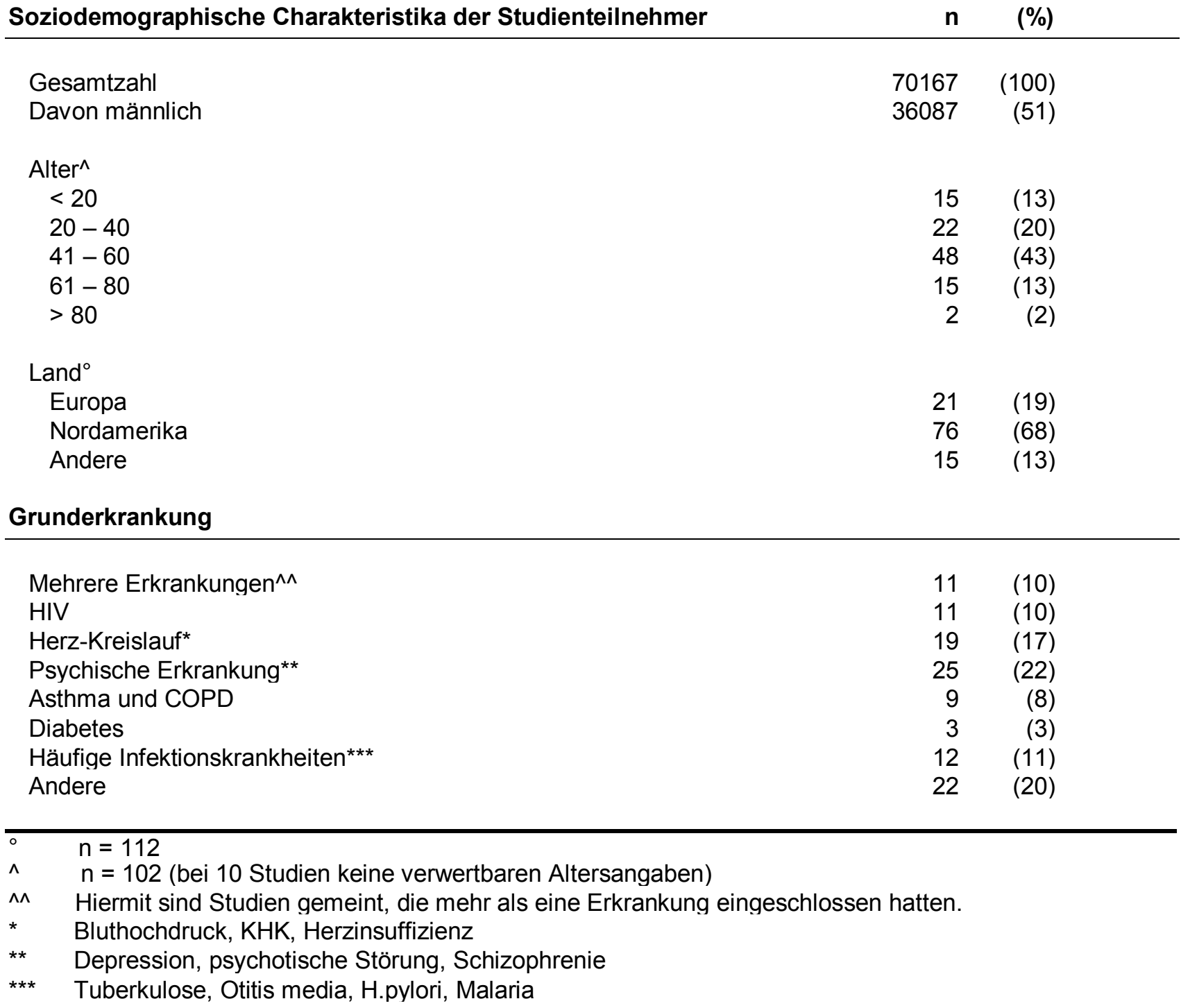

\subsubsection{Adhärenzmessung}

In den 112 Studien wurden insgesamt 278 Methoden verwendet, um Adhärenz zu messen - durchschnittlich rund 2,5 Messmethoden (SD 0,92, Range 2-6) pro Studie. Knapp ein Drittel der Studien (32/112) nutzte mehr als 2 Messmethoden (Tabelle 8). Die durchschnittliche Studiendauer betrug 224,9 Tage (SD 296,7; Range 2-2160). Fast 60 \% (64/110) der Studien dauerten zwischen 20-180 Tage (Tabelle 8). 
Tabelle 8 Daten zur Adhärenzmessung

Charakteristika der Adhärenzmessung

n $\quad(\%)$

\begin{tabular}{|c|c|c|}
\hline \multicolumn{3}{|l|}{ Anzahl der Instrumente* } \\
\hline 2 & 80 & (71) \\
\hline 3 & 19 & (17) \\
\hline 4 & 6 & (5) \\
\hline 5 & 5 & (5) \\
\hline 6 & 2 & (2) \\
\hline \multicolumn{3}{|l|}{ Instrumente ${ }^{\circ}$} \\
\hline Medication Count/Weighing & 57 & (21) \\
\hline Self-Report & 88 & (32) \\
\hline EDM & 21 & (8) \\
\hline Laborbestimmung & 30 & (11) \\
\hline Kollateralreport & 24 & (9) \\
\hline Refill/Medical Record & 25 & (9) \\
\hline Krankheitsbezogene Parameter & 6 & (2) \\
\hline Andere & 27 & (10) \\
\hline \multicolumn{3}{|l|}{ Messung $^{\circ}$} \\
\hline Quantitativ & 144 & (52) \\
\hline Qualitativ & 94 & (34) \\
\hline Unklar & 40 & (14) \\
\hline \multicolumn{3}{|l|}{ Cut-Offs zu Adhärenz definiert* } \\
\hline $\mathrm{Ja}$ & 38 & (34) \\
\hline Nein & 64 & (57) \\
\hline Unklar & 10 & (9) \\
\hline \multicolumn{3}{|c|}{ Erfassung von objektivierbaren Krankheitsoutcomes* } \\
\hline $\mathrm{Ja}$ & 67 & $(60)$ \\
\hline Nein & 45 & (40) \\
\hline \multicolumn{3}{|l|}{ Studiendauer* } \\
\hline$<20$ & 13 & (12) \\
\hline $20-180$ & 64 & (57) \\
\hline$>180$ & 32 & (29) \\
\hline Unklar & 3 & (3) \\
\hline
\end{tabular}

* $\mathrm{n}=112$ eingeschlossene Studien

- $\mathrm{n}=278$ verwendete Messinstrumente

Am häufigsten wurde die Adhärenz per Self-Report gemessen (32 \%), gefolgt von Medication Counts/Weighings. Dabei erfolgte die Messung in knapp mehr als der Hälfte der Studien quantitativ. Etwa ebenso häufig (60 \%) erfassten die Studien objektivierbare Krankheitsoutcomes (Tabelle 8). 
Cut-off-Werte zur Bestimmung der Adhärenz (adhärent ja/nein) wurden nur in einem Drittel der Fälle (38/112) verwendet, und zumeist sehr unterschiedlich definiert (Tabelle 8$)$.

\subsubsection{Adhärenzdefinition in den Studien}

Adhärenzdefinitionen wurden häufig nicht erläutert und oft wurden die Begriffe von Medikamentenadhärenz und Terminadhärenz vermischt (siehe 5.4 2, 6, 14, 48, 73, 95, 96) ${ }^{11}$. Cut-off-Grenzen waren in nur ca. einem Drittel der Studien angegeben (siehe 5.4 5, 10, $11,13,15,20,22,24,26,27,28,30,32,33,39,42,44,51,52,54,55,57,63,64,65,71,72,75,78,86,91,93,94,95,103$, 104, 108, und 112) und zumeist sehr unterschiedlich definiert. Die untere Cut-off-Grenze (der Wert, ab dem ein Patient als adhärent bezeichnet wurde) begann bei $33 \%$ und ging bis $100 \%$. Nur bei 4 dieser 38 Studien fanden sich Obergrenzen von Adhärenz. Die obere Cut-off-Grenze (der Wert, ab dem ein Patient als nicht mehr adhärent bezeichnet wurde) schwankte in diesen Studien zwischen $100 \%$ und $130 \%$. In einer Studie waren die Cut-off-Grenzen für verschiedene Messmethoden unterschiedlich (siehe 5.4 72). Dies begründeten die Autoren damit, dass z.B. Self-Reports zu einer „Überschätzung“ von Adhärenz führen und dass deswegen hier auch ein höherer Cut-off-Wert nötig ist. In einer weiteren Studie wurde im Nachhinein mit verschiedenen Cut-off-Werten die Adhärenz analysiert (siehe 5.4 52).

\subsubsection{Beschreibung der Adhärenzmessung}

Die Beschreibung der Adhärenzmessung war in den meisten Studien nicht sehr detailliert und in 24 Studien fehlten sogar mehrere Informationen zur Durchführung der Adhärenzmessung (siehe 5.4 1, 3, 4, 9, 15, 23, 32, 37, 38, 39, 43, 56, 59, 64, 75, 79, 80, 81, 82, 97, 98, 102, 105 und 106), z.B. Aussagen über Zeitpunkt, Länge oder über die genaue Durchführung der Messungen. Hierdurch war dann nicht erkennbar oder zweifelhaft, ob es eine Baseline-Messung gab, ob bei allen Gruppen die gleichen Messmethoden verwendet wurden, ob die Messungen angekündigt waren und an welchen Zeitpunkten bzw. über welche Zeiträume gemessen wurde. Messhäufigkeit und Messzeitpunkte zeigten so große Unterschiede, dass wir keine nähere Auswertung durchführten.

\footnotetext{
11 In Kapitel 5.4 sind alle 112 eingeschlossenen Studien aufgeführt. Diese Studien werden in Zahlenform zitiert und können dadurch identifiziert werden.
} 
In den Ergebnisteilen mancher Studien wurde von Schwierigkeiten in der Adhärenzerhebung z.B. bei Laborbestimmung oder Medication Counts/Weighings gesprochen, weil Patienten nicht zu ihrem vereinbarten Termin erschienen oder zu Hause nicht anzutreffen waren. Nur wenige Studien beschreiben das Vorgehen in diesen Fällen.

\subsubsection{Präsentation der Ergebnisse}

In 12 Studien wurden die Ergebnisse der einzelnen Adhärenzmesswerkzeuge nicht getrennt aufgeführt (siehe 5.4 3, 22, 24, 56, 59, 88, 90, 91, 101, 105, 110 und 111). Hier wurde dann entweder nur der Wert eines Messinstruments angegeben oder es wurde ein Gesamt-Adhärenzwert aus den verschiedenen Messinstrumenten gebildet. In einer Studie nutzten die Autoren den Begriff der „primären Messmethode“ und die anderen Messmethoden dienten nur der Validierung.

In 3 Studien wurden so genannte „composite index“-Formeln verwendet, in denen Messmethoden zu verschiedenen Prozentzahlen gewertet wurden (z.B. Self-Report mit $20 \%$ und Medication Count/Weighing mit 80 \%; siehe 5.4 3, 22 und 67). Die Entwicklung dieser Formeln war nicht nachvollziehbar. Außerdem waren die Ergebnisse der Messmethoden in 2 der 4 Studien nicht einzeln aufgeführt (siehe 5.4 und 22).

\subsubsection{Adhärenz-Outcome}

Am häufigsten wurde Adhärenz in einem prozentualen Verhältnis angegeben (z.B. eingenommene Medikamente im Vergleich zu verschriebenen Medikamenten oder mit Medikamente abgedeckte Tage pro Monat). Dichotome und kategoriale Angaben bzw. Angaben in Form einer mehrstufigen Skala waren jedoch auch häufig.

\subsubsection{Schlussfolgerungen zu Adhärenzmessung in den betreffenden Studien}

Die Durchführung der einzelnen Messmethoden und ihre Darstellung, die Beschreibung der Adhärenzdefinitionen sowie die Ergebnispräsentation und das AdhärenzOutcome waren sehr heterogen. Dies führt zu einer eingeschränkten Vergleichbarkeit der Ergebnisse. Somit sind auch Vergleiche zwischen den Studien kritisch zu bewerten. 


\subsubsection{Einzelne Messverfahren ${ }^{12}$}

\subsubsection{EDM}

EDM (Electronic Drug Monitoring) wurde bei Medikamenten in Tabletten-/Pillenform aber auch bei Medikamenten in Sprühform verwendet. Das Prinzip ist bei beiden gleich.

Zeitfenster, die festlegen in was für einem zeitlichem Rahmen die Einnahme erfolgen muss, damit der Patient als adhärent eingestuft wurde, definierten die Autoren im Regelfall nicht oder sehr unterschiedlich. Während z.B. in zwei Studien nur kontrolliert wurde, ob die Patienten den Medikamentenbehälter so häufig wie verschrieben geöffnet haben (siehe 5.418 und 35), wurde in 4 Studien außerdem noch überprüft, ob ein bestimmtes Zeitfenster eingehalten wurde (siehe 5.4 41, 74, 83 und 89). In einer weiteren Studie wurde ein Zeitfenster definiert und zusätzlich die Zeitabweichung vom Zeitfenster ermittelt (siehe $\mathbf{5 . 4}$ 60).

Einige Autoren definierten mehrere Öffnungen in einem bestimmten Zeitraum als eine Öffnung. Dies verhindert Verfälschungen der Adhärenz durch so genanntes Curiosity Opening (siehe Fußnote 5, S.7). In einem Großteil der Studien wurde dagegen nicht beschrieben, wie mit mehrfachen Öffnungen innerhalb eines Zeitraums umgegangen wurde. Um den Fehler durch Curiosity Opening weiter zu minimieren, wird empfohlen, ein EDM mit einem Medication Count/Weighing zu kombinieren und nach der korrekten Nutzung des EDM zu fragen. Ein mit dem EDM verbundener Medication Count/Weighing fand sich nur in 10 von 21 Studien (siehe 5.4 21, 41, 42, 47, 52, 59, 60, 68, $74,107)$.

Um Verzerrung durch Pocket Dosing (siehe Fußnote 6, S.7) zu minimieren, sollte ebenfalls ein Medication Count/Weighing durchgeführt werden und nach der korrekten Nutzung des EDM gefragt werden. Diese Frage wurde nur in einer Studie gestellt (siehe 5.4 89).

\footnotetext{
${ }^{12}$ Die einzelnen Verfahren sind in Kapitel 2.2 (siehe auch Tabelle 1, S.7) beschrieben.
} 
In einer Studie wurden die Daten aus dem EDM dem Patienten gezeigt. In diesem Zusammenhang könnte man die Messung zugleich als eine Adhärenz verbessernde Intervention bezeichnen (siehe 5.4 108).

\subsubsection{Self-Reports}

Self-Reports machten einen Großteil der Messmethoden in den untersuchten Studien aus. Auch hier wurden oft wichtige Informationen zur Durchführung nicht beschrieben. So wurde z.B. häufig die Verwendung eines Self-Reports erwähnt - nicht aber, ob es sich dabei um einen Fragebogen, ein Interview, eine Visuell-AnalogSkala oder ein Tagebuch handelte (siehe 5.4 1, 7, 13, 21, 23, 26, 32, 42, 43, 47, 49, 52, 58, 62, 63, 64, 67, 69, 70, 74, 78, 79, 80, 81, 82, 85, 87, 89, 95, 97, 99, 103, 106 und 109). Der Befragungszeitraum (der Zeitraum, über den nach der Adhärenz gefragt wird) spielt bei Self-Reports eine große Rolle und war in den untersuchten Studien sehr unterschiedlich (zwischen 1 Tag und 3 Monaten).

Beim Fragebogen und Interview wurden häufig nicht die Fragen bzw. die Quelle der verwendeten Fragen angegeben. In einem Großteil wurden nicht-validierte Fragebögen/Interviews ohne Angabe der Fragen bzw. der Quelle verwendet (siehe 5.4 3, 5, 7, 9, $10,13,16,24,26,31,36,43,44,45,46,47,49,51,54,56,58,63,69,70,75,78,79,80,81,82,85,95,97,98,99,103,106$ und 109). Unter den validierten Fragebögen fand sich überwiegend der Fragebogen von Morisky et al. (1986; siehe 5.4 6, 25, 28, 31, 52, 102 und 110) und der AIDS Clinical Trials Group Self-Report Questionnaire (Chesney et al. 2000a; siehe 5.4 4, 52, und 91). Weitere verwendete Fragebögen waren der Diabetes Management Scale (Schilling et al. 2002), das Twenty-Four Hour Recall Interview (Freund et al. 1991, Johnson SB et al.1986; siehe 5.4 29) und der Brief Medication Questionnaire (Svarstad et al. 1999; siehe 5.4 83).

Häufig fehlten in den Studien Aussage darüber, ob die Fragen in einer nicht wertenden Form und nicht direkt vom behandelten Personal gestellt wurden, um den Social Desirability Bias (siehe Fußnote 3, S.7) zu minimieren. In den aufgeführten Fragen wurde eher nach nicht eingenommenen (vergessenen) Dosen gefragt als nach ein- 
genommenen Dosen. Das Computer Assisted Self-Interview ${ }^{13}$ wurde nur einmal angewendet (siehe 5.4 81).

\subsubsection{Medication Count/Weighing}

Medication Counts/Weighings wurden auf verschiedene Arten durchgeführt. Meistens wurden die Tabletten, die in bestimmten Gefäßen enthalten waren, gezählt oder aber es wurden spezielle Blisterpacks verwendet, die eine leichtere Abzählung ermöglichen und durch die sich außerdem Rückschlüsse auf den Termin der Nichteinnahme ziehen lassen. Flüssigkeiten/Cremes/Sprays wurden gewogen oder in einer Studie mit einer „genormten“ Spritze gemessen (siehe 5.4 76). Dabei wurde das Ergebnis praktisch immer zu der verschriebenen Dosis in Korrelation gesetzt und daraus ein prozentualer Wert gebildet ( 9 von 10 Tabletten wurden eingenommen $\rightarrow 90$ \% Adhärenz). In einer Studie wurde jedoch keine prozentuale Darstellung, sondern eine kategoriale Darstellung gewählt (siehe 5.4 45)

Bei 3 Studien wurden unangekündigte Medication Counts/Weighings durchgeführt (siehe 5.4 8, 49, 86). In den meisten Studien war jedoch keine ausdrückliche Bemerkung, ob ein Medication Count/Weighing angekündigt oder unangekündigt durchgeführt wurde. In einer Studie wurde der Medication Count/Weighing von den Eltern durchgeführt (siehe 5.4 8). Da die Eltern sich möglicherweise für die Non-Adhärenz ihrer Kinder verantwortlich fühlen, ist hier eine Verzerrung durch den Social Desirability Bias (siehe Fußnote 3, S.7) denkbar. Nur in wenigen Studien wird auf das weitere Vorgehen eingegangen, wenn ein Medication Count/Weighing nicht durchgeführt werden konnte (z.B. weil der Patient nicht zu einem vereinbartem Termin erschien oder er zu Hause nicht anzutreffen war). In einer Studie ersetzte dann z.B. ein SelfReport das Medication Count/Weighing (siehe 5.4 72).

In einer anderen Studie konnte die Baseline-Adhärenzmessung nicht verwertet werden, da vorab nicht geklärt wurde, ob noch andere Medikamentenvorräte existierten

\footnotetext{
${ }^{13}$ Das Computer Assisted Self-Interview ermöglicht einmal die Untersuchung der Adhärenz bei Analphabeten und minimiert zudem ein potentiell stigmatisierendes Verhalten durch den Interviewer und den Social Desirability Bias (siehe Fußnote 3, S.7), da das Interview anonymisiert durchgeführt wird (Johnson AM et al. 2001, Macalino et al. 2002, Metzger et al. 2000).
} 
(siehe 5.4 80). Diese Medikamentenvorräte können nämlich unter Umständen von den Patienten interferierend zu ihren ausgegebenen Medikamenten eingenommen werden und die Genauigkeit des Medication Counts/Weighings stören.

\subsubsection{Kollateralreports}

Die Auswertung der Kollateralreports wird ebenfalls sehr heterogen durchgeführt. Es wurden Eltern, Familienangehörige, Freunde, Pflegepersonal, Apotheker, Ärzte, manchmal auch mehrere Personen der beschriebenen Gruppen nach der Adhärenz eines Patienten befragt. Die Befragung wurde - ähnlich wie beim Self-Report - meist nicht ausführlich beschrieben. Manchmal wurden die gleichen Fragen wie bei dem in der Studie durchgeführten Self-Report verwendet, nur dass das Wort „Ich“ mit dem Wort „Der Patient“ ausgetauscht wurde (siehe 5.4 48). In einigen Fällen fand aber auch keine direkte Befragung statt, sondern es wurden z.B. die Notizen von Krankenschwestern in medizinischen Daten ausgewertet (siehe 5.4 35). Eine andere Studie hat verschiedene Informationsquellen (Labor, Personalangaben, etc.) gesammelt und von einer unbeteiligten dritten Person auswerten lassen (siehe 5.4 69). Die Übergänge zum Refill/Medical Record sind hierbei fließend.

\subsubsection{Refill/Medical Records}

Refill/Medical Records zeigen eine noch größere Heterogenität in ihrer Durchführung - vielleicht die größte der ganzen Messmethoden. In den betreffenden Studien wurden Rezepte, Versicherungsdaten, Apothekendaten, medizinische Krankenakten oder andere Daten ausgewertet, um Rückschlüsse auf die Adhärenz eines Patienten zu ziehen.

Die Studien unterscheiden sich neben der Datenquelle auch in ihrem Auswertungszeitraum. Eine Studie untersuchte Daten aus einem ganzen Jahr (siehe 5.4 33), während bei anderen Studien monatliche Daten ausgewertet wurden. Hier werden unterschiedliche Intervalle für die Definition von Adhärenz gewählt, die jedoch in ihren Ergebnissen nur eingeschränkt vergleichbar sind. Einige Studien konzentrieren sich auf die Abdeckung mit Medikamenten über einen Zeitraum und definieren eine noch „akzeptierte“ Medikamentenlücke (z.B. von 90 Tagen müssen 83 Tage abgedeckt sein). Sie betrachten aber nicht den zeitlichen Zusammenhang dieser fehlenden Tage. Andere Studien messen auch den zeitlichen Zusammenhang und erkennen damit Drug 
Holidays (Zeitabschnitte, in denen Medikamente nicht eingenommen werden). In einer Studie wurde zusätzlich die Adhärenz ein halbes Jahr vor Studienbeginn untersucht, um eine verlässlichere Aussage über die Adhärenzänderung zu machen (siehe 5.4 52).

Bei Refill/Medical Records ist es wichtig, welche Medikamentendosis der Adhärenzmessung zu Grunde gelegt wird. In den untersuchten Studien gab es hierzu zwei Methoden. In einem Teil der Studien wurde eine Dosis z.B. durch die Agency For Health Care Policy And Research Guidelines festgelegt. Die Wirksamkeit des Medikaments ist erst ab dieser Mindestdosis gesichert und der Patient wird als adhärent angesehen, solange seine Einnahmedosis über jener festgelegten Mindestdosis liegt (siehe 5.4 55, 56 und 57). Andere Studien haben die eingenommene Dosis mit der verschriebenen Dosis verglichen und der Patient ist dann non-adhärent, wenn er nicht diese Dosis einnimmt. Einige dieser Patienten wären aber evtl. nach der erstbeschriebenen Methode noch adhärent.

Nur eine Studie widmet sich der Frage nach Medikamentenvorräten, die eine mögliche Fehlerquelle für die Adhärenzmessung anhand des Refill/Medical Record sein können (siehe 5.4 28).

Problematisch ist eine nur kurzfristige Anwendung des Refill/Medical Records bei Medikamenten, deren richtige Dosierung eine längere Einstellung erfordert. In einer Studie wurde die Adhärenz bei Immunsuppression untersucht. Dort wurden Adhärenzschwankungen in den ersten 3 Monaten auf Dosismodifikationen zurückgeführt (siehe 5.4 22). Adhärenzprobleme waren in dieser Zeit nicht feststellbar.

\subsubsection{Laborbestimmungen}

In den ausgewerteten Studien wurde im Urin oder im Serum entweder das Medikament selber, ein Metabolit oder ein beigemischter Stoff, der leichter nachzuweisen ist (siehe 5.4 75), bestimmt. Man muss auch bei dieser Messmethode wieder zwischen angekündigten und unangekündigten Messungen (der Patient ist über Blutabhnahme, Urinprobe o.ä. informiert oder nicht) unterscheiden. Angekündigte Messungen sind anfälliger für eine Überbewertung der Adhärenz. 
Die meisten Studien haben die Laborbestimmung nur stichprobenmäßig angewendet, um diese Ergebnisse als Validierungsreferenz für andere Messmethoden zu verwenden. In 6 Studien wurde die Probe rein auf das Vorhandensein des Medikaments, Metaboliten oder beigemischten Stoffs bestimmt (siehe 5.4 21, 49, 53, 66, 70 und 109). In den anderen Studien wurden vorher zu erreichende Wirkstoffspiegel festgelegt. Einige Studien haben hierbei von ihren Patienten am Anfang der Studie „Metabolisierungsprofile“ erstellt, um Verzerrungen durch Unterschiede in der Verstoffwechselung möglichst gering zu halten (siehe $\mathbf{5 . 4} 62$ und 87). Nur in einer Studie wurde beschrieben, wie mit „nicht durchführbaren“ Messungen umgegangen wurde (siehe $5.462)$.

\subsubsection{Krankheitsbezogene Parameter}

In $60 \%$ der Studien wurden objektivierbare Krankheitsoutcomes bestimmt. Allerdings nutzen nur $2 \%$ der Studien diese zur Bestimmung der Adhärenz. Als Krankheitsparameter wurde je nach Krankheit und Behandlung TSH, Gerinnungsparameter, Blutdruck, HbA1c-Werte oder spezielle Zellwerte (Bsp. Anzahl der CD4+-Zellen bei HIV oder das mittlere korpuskuläre Erythrozytenvolumen bei Behandlung mit einem Chemotherapeutikum) bestimmt (siehe 5.4 14, 25, 73, 84, 111).

\subsubsection{Andere Messmethoden}

Mit anderen Messmethoden sind Verfahren gemeint, die sich nicht in die oben genannten Hauptgruppen einordnen lassen. Dies liegt zum einen daran, dass sie vielleicht eine grenzwertige Messmethode sind oder an sich schon wieder als Intervention angesehen werden können (wie z.B. Directly Observed Therapy [DOT], Termineinhaltungen, Spezialkalender oder Medikamentenumstellung). Zum anderen handelt es sich um Messungen, die nur unter bestimmten Krankheits- bzw. Umfeldbedingungen anzuwenden sind (z.B. Observational Checklist bei Asthma). 


\subsubsection{Messmethoden im Studienkontext}

\section{Anzahl der Messmethoden und Instrumente}

Tabelle 9 und 10 stellen den Zusammenhang zwischen Messmethoden und Anzahl der Messmethoden dar. Studien, die 2 Messmethoden verwendet haben, nutzten in 39 \% einen Self-Report, in $12 \%$ Refill/Medical Records und in $9 \%$ EDM. Studien mit 3 Messmethoden verwendeten hingegen nur in $23 \%$ einen Self-Report, in $5 \%$ Refill/Medical Records und in $4 \%$ EDM. Laborbestimmungen und Medication Counts/Weighings veränderten ihre Häufigkeit praktisch nicht. Kollateralreports wurden etwa doppelt so häufig und krankheitsbezogene Parameter fast 6-mal so häufig genutzt.

Da nur 13 Studien mehr als 3 Messmethoden nutzten, sind verlässliche Aussagen über deren Verteilung letztlich nicht möglich. Messinstrumente wie Kollateralreports, verschiedene andere Messmethoden oder Laborbestimmungen wurden vor allem in Studien verwendet, die mehrere Messmethoden nutzten.

Tabelle 9 Instrumente und Anzahl der Messmethoden

\begin{tabular}{|c|c|c|c|c|c|c|c|c|c|}
\hline & \multicolumn{9}{|c|}{ Typ der Messung; n (\%) } \\
\hline & EDM & Self-Report & $\begin{array}{l}\text { Kollateral- } \\
\text { report }\end{array}$ & $\begin{array}{c}\text { Medication } \\
\text { Count/ } \\
\text { Weighing }\end{array}$ & $\begin{array}{l}\text { Labor- } \\
\text { bestim- } \\
\text { mung }\end{array}$ & $\begin{array}{c}\text { Pharmacy/ } \\
\text { Medical } \\
\text { Record }\end{array}$ & $\begin{array}{c}\text { Krank- } \\
\text { heitsbez. } \\
\text { Parameter }\end{array}$ & Andere & Alle \\
\hline \multicolumn{10}{|l|}{ Anzahl der } \\
\hline \multicolumn{10}{|l|}{ Messmethoden } \\
\hline $\begin{array}{l}\text { Zahl der } \\
\text { Messmethoden; n }\end{array}$ & 21 & 88 & 24 & 57 & 30 & 25 & 6 & 27 & 278 \\
\hline $\begin{array}{l}\varnothing \text { Messmethoden; } \\
\mathrm{n}(\mathrm{SD})\end{array}$ & $2,7(1,1)$ & $2,5(1,0)$ & $2,9(1,3)$ & $2,6(0,9)$ & $2,9(1,2)$ & $2,5(1,1)$ & $2,6(0,5)$ & $3,7(1,1)$ & $2,5(0,9)$ \\
\hline \multicolumn{10}{|l|}{ Art der Erfassung } \\
\hline Qualitativ; n (\%) & - & $43(49)$ & $12(50)$ & 1 (2) & $16(53)$ & $7(28)$ & $3(50)$ & $12(44)$ & $94(34)$ \\
\hline Quantitativ; n (\%) & $20(95)$ & $27(31)$ & $8(33)$ & $51(90)$ & $12(40)$ & $16(64)$ & $1(17)$ & $9(33)$ & $144(52)$ \\
\hline Unklar; n(\%) & 1 (5) & $18(21)$ & $4(17)$ & $5 \quad(9)$ & $2 \quad(7)$ & $2(8)$ & $2(33)$ & $6(22)$ & $40(14)$ \\
\hline
\end{tabular}


In den Studien überwiegt die quantitative Adhärenzmessung (52\% vs. 34 \%). EDM, Medication Count/Weighing und Refill/Medical Record haben Adhärenz dabei überwiegend quantitativ gemessen, während Self-Report, Kollateralreport, Laborbestimmungen, krankheitsbezogene Parameter und verschiedene andere Messmethoden Adhärenz eher qualitativ gemessen haben.

Tabelle 10 Anzahl der Messmethoden und Instrumente

\begin{tabular}{|c|c|c|c|c|}
\hline & \multicolumn{4}{|c|}{ Anzahl der Messmethoden } \\
\hline & 2 & 3 & $>3$ & Alle \\
\hline Zahl der Studien; n (\%) & $80(71)$ & $19(17)$ & $13(12)$ & $112(100)$ \\
\hline \multicolumn{5}{|l|}{ Typ der Messung* } \\
\hline EDM; n (\%) & $14 \quad(9)$ & $2 \quad(4)$ & $5 \quad(8)$ & 21 \\
\hline Self-Report; n (\%) & $62(39)$ & $13(23)$ & $13(21)$ & $88 \quad(32)$ \\
\hline Kollateralreport; n (\%) & $9 \quad(6)$ & $6(11)$ & $9 \quad(15)$ & 24 \\
\hline Med. Count/Weighing; n (\%) & $36(23)$ & $13(23)$ & $8 \quad(13)$ & $57 \quad(21)$ \\
\hline Laborbestimmung; n (\%) & $16(10)$ & $7(12)$ & $7 \quad(12)$ & $30 \quad(11)$ \\
\hline Refill/Medical Record; n (\%) & $19(12)$ & $3 \quad(5)$ & $3 \quad(5)$ & 25 \\
\hline Krankh. Parameter; n (\%) & $2(1)$ & $4 \quad(7)$ & $0 \quad(0)$ & (2) \\
\hline Andere; n (\%) & $2 \quad(1)$ & $9(16)$ & $16(26)$ & $27 \quad(10)$ \\
\hline
\end{tabular}

* Insgesamt 278 Messungen in 112 Studien 
Bei Patienten über 60 Jahren wurden durchschnittlich weniger Messmethoden (im Schnitt 2,0 Messmethoden) angewendet als bei den anderen Altersgruppen (Tabelle 11).

Auch die Verteilung der Messmethoden war altersabhängig. Subjektive Messmethoden wie Self-Reports wurden bei Kindern und Personen über 60 weniger genutzt, Medication Counts/Weighings dagegen häufiger (Tabelle 11). EDM wurde praktisch erst bei Patienten ab dem 21. Lebensjahr verwendet und besonders in den Altersgruppen 41 bis 60 bzw. 61 bis 80 . Refill/Medical Records wurden proportional mit steigendem Alter vermehrt eingesetzt, wohingegen Laborbestimmungen besonders häufig bei Patienten unter 21 verwendet wurden (Tabelle 11).

Tabelle 11 Messmethode, Anzahl der Messmethoden und Alter der Studienteilnehmer

\begin{tabular}{cccccccc}
\hline \multicolumn{7}{c}{ Alter der Studienteilnehmer; in Jahren } \\
\cline { 2 - 6 } & $1-20$ & $21-40$ & $41-60$ & $61-80$ & $>80$ & Alle \\
\hline
\end{tabular}

\section{Anzahl der Messmethoden}

$\begin{array}{lrlllllllllll}\text { Zahl der Studien; n (\%) } & 15 & (15) & 22 & (22) & 48 & (47) & 15 & (15) & 2 & (2) & 102^{*} & (100) \\ \varnothing \text { Messmethoden; n (SD) } & 2,5 & (0.7) & 2,6 & (1,1) & 2,5 & (1,0) & 2 & (0) & 2 & (0) & 2,5 & (0,8)\end{array}$

Typ der Messung**

$\begin{array}{lrrrrrrrrrrrr}\text { EDM; n (\%) } & 1 & (3) & 3 & (5) & 13 & (11) & 4 & (13) & 0 & (0) & 21 & (8) \\ \text { Self-Report; n (\%) } & 9 & (24) & 20 & (35) & 46 & (38) & 8 & (27) & 1 & (25) & 85 & (33) \\ \text { Kollateralreport; n (\%) } & 6 & (16) & 12 & (21) & 3 & (3) & 1 & (3) & 0 & (0) & 24 & (9) \\ \text { Med. Count/Weighing;n (\%) } & 10 & (26) & 7 & (12) & 24 & (20) & 8 & (27) & 2 & (50) & 51 & (20) \\ \text { Laborbestimmung; n (\%) } & 7 & (18) & 7 & (12) & 11 & (9) & 2 & (7) & 0 & (0) & 28 & (11) \\ \text { Refill/Medical Record; n (\%) } & 2 & (5) & 4 & (7) & 12 & (10) & 5 & (17) & 0 & (0) & 23 & (9) \\ \text { Krankh. Parameter; n (\%) } & 0 & (0) & 0 & (0) & 2 & (2) & 1 & (3) & 1 & (25) & 4 & (2) \\ \text { Andere; n (\%) } & 3 & (8) & 4 & (7) & 11 & (9) & 1 & (3) & 0 & (0) & 19 & (8)\end{array}$

\footnotetext{
* In 10 der 112 Studien haben die Autoren keine verwertbaren Aussagen über die Altersverteilung gemacht

** $\mathrm{N}=251$; Mehrfachnennungen
} 
Landesspezifische Unterschiede in der Adhärenzmessung werden in Tabelle 12 dargestellt. In Nordamerika angefertigte Studien verwendeten mehr Messmethoden als Studien in anderen Kontinenten, vor allem weit häufiger Refill/Medical RecordsMessungen, ebenso Laborbestimmungen. Hingegen wurde EDM besonders in Europa genutzt. In den 15 Studien aus anderen Ländern gab es keine EDM-Verwendung. Dafür wurden hier sehr viel häufiger Medication Counts/Weighings durchgeführt. Self-Reports und Kollateralreports wiesen nur moderate landesspezifische Unterschiede aus.

Tabelle 12 Messmethode, Anzahl der Messmethoden und Land der Studie

\begin{tabular}{|c|c|c|c|c|c|c|c|c|}
\hline & \multicolumn{8}{|c|}{ Land } \\
\hline & \multicolumn{2}{|c|}{ Nordamerika } & \multicolumn{2}{|c|}{ Europa } & \multicolumn{2}{|c|}{ Andere } & \multicolumn{2}{|c|}{ Alle } \\
\hline \multicolumn{9}{|l|}{ Anzahl der Messmethoden } \\
\hline Zahl der Studien; n (\%) & 76 & $(68)$ & 21 & (19) & 15 & $(13)$ & 112 & $(100)$ \\
\hline$\varnothing$ Messmethoden; n (SD) & 2,6 & (1) & 2,2 & $(0,6)$ & 2,1 & $(0,4)$ & 2,5 & $(0,9)$ \\
\hline \multicolumn{9}{|l|}{ Typ der Messung* } \\
\hline EDM; n (\%) & 16 & (8) & 5 & $(11)$ & 0 & $(0)$ & 21 & (8) \\
\hline Self-Report; n (\%) & 63 & $(32)$ & 14 & $(30)$ & 11 & $(34)$ & 88 & (32) \\
\hline Kollateralreport; $\mathrm{n}(\%)$ & 16 & (8) & 5 & $(11)$ & 3 & (9) & 24 & (9) \\
\hline Med. Count/Weighing; n (\%) & 34 & $(17)$ & 11 & $(24)$ & 12 & $(38)$ & 57 & (21) \\
\hline Laborbestimmung; n (\%) & 24 & $(12)$ & 3 & (7) & 3 & (9) & 30 & (11) \\
\hline Refill/Medical Record; n (\%) & 21 & $(11)$ & 3 & (7) & 1 & (3) & 25 & (9) \\
\hline Krankh. Parameter; n (\%) & 5 & (3) & 1 & $(2)$ & 0 & $(0)$ & 6 & (2) \\
\hline Andere; n (\%) & 21 & $(11)$ & 4 & (9) & 2 & (6) & 27 & $(10)$ \\
\hline
\end{tabular}


Die durchschnittlich verwendete Anzahl der Messmethoden in den Studien hing auch von der Grunderkrankung ab. Vor allem bei psychischen Erkrankungen, Asthma/COPD und Infektionskrankheiten wurden mehr Messmethoden verwendet (Tabelle 13). EDM wurde vor allem bei HIV eingesetzt, Kollateralreports bei psychischen Erkrankungen und Self-Reports bei Adhärenzinterventionsstudien, die mehrere Krankheiten eingeschlossen hatten (Tabelle 13).

Tabelle 13 Messmethode, Anzahl der Messmethoden und Einschlussdiagnose

\begin{tabular}{|c|c|c|c|c|c|c|c|c|c|c|}
\hline & \multicolumn{10}{|c|}{ Grunderkrankung } \\
\hline & $\begin{array}{c}\text { Mehrere } \\
\text { Erkrankun- } \\
\text { gen }\end{array}$ & & IIV & $\begin{array}{l}\text { Herz- } \\
\text { Kreislauf }\end{array}$ & $\begin{array}{l}\text { Psychische } \\
\text { Erkrankun- } \\
\text { gen }\end{array}$ & $\begin{array}{l}\text { Asthma und } \\
\text { COPD }\end{array}$ & Diabetes & $\begin{array}{c}\text { Häufige- } \\
\text { Infektions- } \\
\text { krankheiten }\end{array}$ & Diverse & Alle \\
\hline \multicolumn{11}{|l|}{ Anzahl der Messmethoden } \\
\hline Zahl der Messmethoden; $\mathrm{n}$ & 11 & 11 & & 19 & 25 & 9 & 3 & 12 & 22 & 112 \\
\hline$\varnothing$ Messmethoden; n (SD) & $2,2 \quad(0.6)$ & 2,5 & $(1,0)$ & $2,5 \quad(0,8)$ & $2,8 \quad(1,3)$ & $2,7 \quad(0,9)$ & $2,3 \quad(0,8)$ & $2,6 \quad(1,0)$ & $2,1 \quad(0,4)$ & $2,5 \quad(0,9)$ \\
\hline \multicolumn{11}{|l|}{ Typ der Messung; n (\%) } \\
\hline EDM & 1 (4) & & 7 (26) & $2 \quad(4)$ & $3 \quad(4)$ & $3(13)$ & $2(29)$ & 1 (3) & $2 \quad(4)$ & $21 \quad(8)$ \\
\hline Self-Report & $11(46)$ & & $2(44)$ & $15(32)$ & $20(28)$ & 7 (29) & $3(43)$ & $7(23)$ & $13(28)$ & $89(32)$ \\
\hline Kollateralreport & - & & - & $4 \quad(9)$ & $12(17)$ & $3(13)$ & $1(14)$ & $3(10)$ & $1 \quad(2)$ & $24 \quad(9)$ \\
\hline Med. Count/Weighing & $8(33)$ & & $4(15)$ & $12(26)$ & $8(11)$ & $3(13)$ & $1(14)$ & $10(32)$ & $11(23)$ & $57(21)$ \\
\hline Laborbestimmung & - & & $1 \quad(4)$ & $3 \quad(4)$ & $7(10)$ & $4(17)$ & - & $7(23)$ & $8(17)$ & $30(11)$ \\
\hline $\begin{array}{l}\text { Pharmacy/Medical } \\
\text { Record }\end{array}$ & $2(8)$ & 2 & $2 \quad(7)$ & $2 \quad(4)$ & $10(14)$ & $1 \quad(4)$ & - & - & $8(17)$ & $25 \quad(9)$ \\
\hline $\begin{array}{l}\text { Krankheitsbez. } \\
\text { Parameter }\end{array}$ & 1 (4) & 1 & 1 (4) & $\begin{array}{ll}4 \quad(9)\end{array}$ & - & - & - & - & 1 (2) & $6 \quad$ (2) \\
\hline Andere & 1 (4) & & $1 \quad$ (4) & $5(11)$ & 11 (16) & 3 (13) & - & $3(10)$ & $3 \quad(6)$ & $27(10)$ \\
\hline
\end{tabular}

\section{Art des Studienaufbaus und Sponsoring}

Die Anwendung von Self-Reports war weitestgehend unabhängig von der Art der Studienaufbaus. EDM unterschied sich hierbei erheblich (20\% bei faktorieller Grup- 
penbildung, $17 \%$ bei Crossover-Gruppenbildung, $7 \%$ bei paralleler Gruppenbildung, $3 \%$ bei anderer Gruppenbildungen; siehe Tabelle 14).

In Tabelle 15 ist der Zusammenhang zwischen Messmethode, Anzahl der Messmethoden und Sponsoring dargestellt. Studien, die allein von einer kommerziellen Organisation gefördert wurden, nutzten im Schnitt weniger Messmethoden. Die Nutzung der verschiedenen Messmethoden war ebenso von der Art der Förderung abhängig. So wurde das EDM bei kombinierter Förderung durch kommerzielle und nicht kommerzielle Organisationen doppelt so häufig angewendet wie bei ausschließlicher Förderung durch eine nicht kommerzielle Organisation.

Tabelle 14 Messmethode, Anzahl der Messmethoden und Art der Gruppenbildung

\begin{tabular}{|c|c|c|c|c|c|c|c|c|c|c|}
\hline & \multicolumn{10}{|c|}{ Art der Gruppenbildung } \\
\hline & \multicolumn{2}{|c|}{ Parallel } & \multicolumn{2}{|c|}{ Cross-over } & \multicolumn{2}{|c|}{ Faktoriell } & \multicolumn{2}{|c|}{ Andere } & \multicolumn{2}{|c|}{ Alle } \\
\hline Anzahl der Messmethoden & & & & & & & & & & \\
\hline Zahl der Studien; n (\%) & 89 & $(80)$ & 3 & (3) & 6 & (5) & 14 & (13) & 112 & $(100)$ \\
\hline$\varnothing$ Messmethoden; n (SD) & 2,5 & $(0,9)$ & 2,0 & $(0)$ & 2,5 & $(1,2)$ & 2,5 & $(0,9)$ & 2,5 & $(0,9)$ \\
\hline \multicolumn{11}{|l|}{ Typ der Messung* } \\
\hline EDM; n (\%) & 16 & $(7)$ & 1 & $(17)$ & 3 & $(20)$ & 1 & (3) & 21 & (8) \\
\hline Self-Report; n (\%) & 70 & $(32)$ & 2 & $(33)$ & 5 & (33) & 11 & (31) & 88 & (32) \\
\hline Kollateralreport; n (\%) & 22 & $(10)$ & 1 & $(17)$ & 0 & $(0)$ & 1 & (3) & 24 & (9) \\
\hline Med. Count/Weighing; n (\%) & 44 & (20) & 1 & $(17)$ & 3 & (20) & 9 & (26) & 57 & (21) \\
\hline Laborbestimmung; n (\%) & 22 & (10) & 0 & $(0)$ & 1 & $(7)$ & 7 & (20) & 30 & (11) \\
\hline Refill/Medical Record; n (\%) & 19 & (9) & 1 & $(17)$ & 2 & $(13)$ & 3 & (9) & 25 & (9) \\
\hline Krankh. Parameter; n (\%) & 6 & (3) & 0 & $(0)$ & 0 & $(0)$ & 0 & $(0)$ & 6 & (2) \\
\hline Andere; n (\%) & 23 & $(10)$ & 0 & $(0)$ & 1 & (7) & 3 & (9) & 27 & (10) \\
\hline
\end{tabular}

${ }^{*} \mathrm{~N}=278$

\section{$\underline{\text { Studiendauer }}$}

Studien bis 20 Tage bzw. mit mehr als 180 Tagen nutzten durchschnittlich weniger Messmethoden als mittellange Studien (Tabelle 16). Studien, die nicht länger als 3 
Wochen dauerten, nutzten häufig Medication Counts/Weighings (41\%), Self-Reports (28\%) und Laborbestimmung (10\%). In diesen Studien wurde kein EDM und nur einmal Refill/Medical Records verwendet (Tabelle 16). Studien über 180 Tage nutzten v.a. Self-Reports (39 \%), Refill/Medical Records (15\%) und Kollateralreports (15 \%). Medication Counts/Weighings, Laborbestimmung bzw. EDM wurden relativ selten verwendet.

Tabelle 15 Messmethode, Anzahl der Messmethoden und Sponsoring

\begin{tabular}{ccccc}
\hline & \multicolumn{3}{c}{ Sponsoring durch } \\
\cline { 2 - 5 } & Kommerz. & Nicht & Kommerz. & \\
Organisation & kommerz. & und nicht & Keine An- & Alle \\
& & Organisation & kommerz. & gaben \\
& & & Organisation & \\
\hline
\end{tabular}

\section{Anzahl der Messmethoden}

\begin{tabular}{|c|c|c|c|c|c|}
\hline Zahl der Studien; n & 17 & 49 & 14 & 32 & 112 \\
\hline$\varnothing$ Messmethoden; n (SD) & $2,2 \quad(0,4)$ & $2,6 \quad(1,0)$ & $2,5 \quad(0,8)$ & $2,4 \quad(1,1)$ & $2,5 \quad(0,9)$ \\
\hline
\end{tabular}

\section{Typ der Messung*}

\begin{tabular}{lrrrrrrrrrr} 
EDM; n (\%) & 4 & $(11)$ & 8 & $(6)$ & 5 & $(14)$ & 4 & $(5)$ & 21 & $(8)$ \\
Self-Report; n (\%) & 13 & $(34)$ & 44 & $(35)$ & 9 & $(26)$ & 22 & $(28)$ & 88 & $(32)$ \\
Kollateralreport; n (\%) & 1 & $(3)$ & 14 & $(11)$ & 4 & $(11)$ & 5 & $(6)$ & 24 & $(9)$ \\
Med. Count/Weighing; n (\%) & 7 & $(18)$ & 21 & $(17)$ & 9 & $(26)$ & 20 & $(26)$ & 57 & $(21)$ \\
Laborbestimmung; n (\%) & 5 & $(13)$ & 13 & $(10)$ & 3 & $(9)$ & 9 & $(12)$ & 30 & $(11)$ \\
Refill/Medical Record; n (\%) & 4 & $(11)$ & 14 & $(11)$ & 0 & $(0)$ & 7 & $(9)$ & 25 & $(9)$ \\
Krankh. Parameter; n (\%) & 1 & $(3)$ & 2 & $(2)$ & 2 & $(6)$ & 1 & $(1)$ & 6 & $(2)$ \\
Andere; n (\%) & 3 & $(8)$ & 11 & $(9)$ & 3 & $(9)$ & 10 & $(13)$ & 27 & $(10)$ \\
\hline
\end{tabular}

${ }^{*} \mathrm{~N}=278$

\section{Anzahl der Messmethoden im zeitlichen Zusammenhang}

Die Anzahl der angefertigten Studien ist in den letzten Jahren stark angestiegen und mehr als ein Drittel der Studien ist in den letzten Jahren entstanden. Die mittlere verwendete Messmethodenanzahl hat sich im Lauf der Zeit wenig verändert. (Tabelle 17). 
Tabelle 16 Messmethode, Anzahl der Messmethoden und Studiendauer

\begin{tabular}{|c|c|c|c|c|c|c|c|c|}
\hline & \multicolumn{8}{|c|}{ Studiendauer; in Tagen } \\
\hline & \multicolumn{2}{|c|}{$<20$} & \multicolumn{2}{|c|}{$21-180$} & \multicolumn{2}{|c|}{$>180$} & \multicolumn{2}{|c|}{ Alle } \\
\hline \multicolumn{9}{|l|}{ Anzahl der Messmethoden } \\
\hline Zahl der Studien; n & 13 & & 64 & & 32 & & $109^{*}$ & \\
\hline$\varnothing$ Messmethoden; n (SD) & 2,2 & $(0,6)$ & 2,6 & $(1,0)$ & 2,3 & $(0,7)$ & 2,5 & $(0,9)$ \\
\hline \multicolumn{9}{|l|}{ Typ der Messung** } \\
\hline EDM; n (\%) & 0 & $(0)$ & 15 & (9) & 6 & (8) & 21 & (8) \\
\hline Self-Report; n (\%) & 8 & $(28)$ & 48 & $(29)$ & 29 & (39) & 85 & (31) \\
\hline Kollateralreport; $n(\%)$ & 3 & $(11)$ & 9 & (5) & 11 & $(15)$ & 23 & (9) \\
\hline Med. Count/Weighing; n (\%) & 12 & (41) & 37 & (22) & 6 & (8) & 55 & (20) \\
\hline Laborbestimmung; n (\%) & 3 & $(10)$ & 20 & $(12)$ & 6 & (8) & 29 & (11) \\
\hline Refill/Medical Record; n (\%) & 1 & (3) & 13 & (8) & 11 & $(15)$ & 25 & (9) \\
\hline Krankh. Parameter; n (\%) & 0 & (0) & 6 & (4) & 0 & (0) & 6 & (2) \\
\hline Andere; n (\%) & 2 & (7) & 20 & $(12)$ & 5 & (7) & 27 & (10) \\
\hline
\end{tabular}

\footnotetext{
* In 3 der 112 Studien haben die Autoren keine verwertbaren Angaben zu Studiendauer gemacht.

** $\mathrm{N}=271$
}

Tabelle 17 Anzahl der Messmethoden im zeitlichen Verlauf

\begin{tabular}{rrrrrrrrr}
\hline \multicolumn{10}{c}{ 5-Jahres-Gruppen } \\
\cline { 2 - 8 } & $1972-$ & $1977-$ & $1982-$ & $1987-$ & $1992-$ & $1997-$ & $2002-$ & Ge- \\
1976 & 1981 & 1986 & 1991 & 1996 & 2001 & 2006 & samt \\
\hline
\end{tabular}

\section{Anzahl der Messmethoden}

Zahl der Studien; $\mathrm{n}$

$\begin{array}{rrrrrrrr}2 & 10 & 14 & 12 & 12 & 20 & 42 & 112 \\ 3,0 & 2,4 & 3,0 & 2,4 & 2,3 & 2,3 & 2,5 & 2,5 \\ (0,0) & (0,5) & (1,4) & (1,0) & (0,7) & (0,7) & (0,9) & (0,9)\end{array}$




\subsection{Qualitätseinschätzung der Studien}

Die Qualitätseinschätzung erfolgte in drei Schritten: (1) Bewertung nach Anzahl und Validität der verwendeten Messmethoden (siehe Kapitel 5.3.1); danach (2) wurden Studien mit 2 Messmethoden genauer anhand weiterer Qualitätskriterien allgemein bewertet und in einer Scorebildung überprüft (siehe Kapitel 5.3.2) und abschließend (3) wurden speziell die häufig verwendeten Messmethoden Self-Report und Medication Count/Weighing evaluiert (siehe Kapitel 5.3.3).

\subsubsection{Qualitätsbewertung aller Studien}

Von 448 eingeschlossenen Studien erfüllten nur 112 Studien (25\%) die Kriterien für die nachfolgende detaillierte Qualitätsbewertung (Tabelle 18). Von den 336 ausgeschlossenen Studien nutzten immerhin fast 90 \% (301/336) eine validierte Messmethode ${ }^{14}$.

Tabelle 18 Bewertung aller Studien anhand der Anzahl und Validität der Messmethoden $(n=448)$

\begin{tabular}{lrr} 
& $\mathbf{n}$ & (\%) \\
\cline { 2 - 3 } Nur eine unvalidierte Messmethode & 35 & $(8)$ \\
Nur eine validierte Messmethode & 301 & $(67)$ \\
Mindestens zwei validierte Messmethoden & 112 & $(25)$
\end{tabular}

\subsubsection{Qualitätsbewertung der Studien mit mindestens 2 Messmethoden}

Von den 112 Studien mit 2 oder mehr Messmethoden nutzten 98 Studien (88\%) mindestens 2 validierte Messmethoden. 3 Studien verwendeten gar keine validierte Messmethode (Tabelle 19).

\footnotetext{
14 Mit validierten Messmethoden sind hier die in der Literatur grundsätzlich anerkannten Messmethoden gemeint (EDM, Self-Report, Medication Count/Weighing, Laborbestimmung und Refill/Medical Record). Es kann hierbei jedoch keine Aussage über die spezielle Validität oder Qualität der in der Studie durchgeführten Messung gegeben werden. DOT, Kollateralreport, Termineinhaltung, krankheitsbezogene Parameter sowie andere nicht näher zu spezifizierende Messmethoden wurden als unvalidierte Messmethoden angesehen.
} 
Ausreichende Aussagen zu formellen Aspekten bzw. die getrennte Ergebnisaufführung fehlten in ca. einem Fünftel der Studien (22 \% bzw. $17 \%$ ). In ca. 40 \% der Studien (46/112) wurden keine objektivierbaren Krankheitsoutcomes bestimmt und in $66 \%$ der Studien waren keine Cut-off-Definitionen zur Adhärenz definiert bzw. es war unklar, ob mit Cut-offs gearbeitet wurde (Tabelle 19).

Der Großteil der Studien untersuchte nur ein Krankheitsbild (91\%) und trennte Termin- und Medikamentenadhärenz (94\%).

Tabelle 19 Detaillierte Auswertung der Studien mit mehr als einer Messmethode $(n=112)$

Anzahl validierter Messmethoden

Keine validierte Messmethode

Eine validierte Messmethode

Mindestens zwei validierte Messmethoden

\section{Messung von objektivierbaren Krankheitsoutcomes}

$\mathrm{Ja}$

Nein

Trennung von Termin-/Medikamentenadhärenz

Ja

Nein

Cut-Offs zu Adhärenz definiert

$\mathrm{Ja}$

Nein

Unklar

Ausreichende methodische Angaben bezüglich Durchführung

Ja

Aufführung der einzelnen Messergebnisse

Ja

Nein

Studie untersucht nur eine Erkrankung 
Der von uns verwendetete Score für die Qualitätseinschätzung der Artikel ist in Kapitel 4.4.2 beschrieben. Mangelhafte bzw. gute Qualität bezüglich der Qualität der Adhärenzmessung war in den Studien mit mindestens 2 Messmethoden in ca. einem Fünftel der Studien gegeben (Tabelle 20).

Tabelle 20 Qualitätsscore bezüglich Adhärenzmessung und ihrer Darstellung

\begin{tabular}{llll}
\hline Qualitätsscore & & $\mathbf{n}$ & (\%) \\
\hline Mangelhaft & $(-2$ bis 3 Punkte) & 27 & $(24)$ \\
Moderat & (4 bis 5 Punkte) & 61 & $(55)$ \\
Gut & (6 bis 7 Punkte) & 24 & $(21)$ \\
\hline
\end{tabular}

\subsubsection{Bewertung von „Self-Reports“ und „Medication Counts/Weighings“}

Der Self-Report war das am häufigsten genutzte Messinstrument in den 112 Studien, die mindestens 2 Messmethoden nutzten (insgesamt 88-mal). Dabei wurden nur in einem kleinen Teil (16\%) Self-Reports verwendet, zu denen Validierungsstudien existieren. In 50 Studien war die Beschreibung der Verwendung des Messinstruments mangelhaft: in 37 Studien fehlte die Beschreibung der Durchführung und in 13 Studien war sogar gänzlich unklar, welche Art des Self-Reports überhaupt durchgeführt wurde (Tabelle 21).

Medication Count/Weighing wurde am zweithäufigsten verwendet ( $N=57)$. Auch hier war die Beschreibung der Durchführung mangelhaft. In fast $90 \%$ (49/57) war nicht festzustellen, ob der Medication Count/Weighing angekündigt oder unangekündigt durchgeführt wurde (Tabelle 21). 
Tabelle 21 Analyse der am häufigsten verwendeten Messmethoden

Self-Report $(\mathrm{N}=\mathbf{8 8})$

Valider Self-Report

Nicht valider Self-Report, Beschreibung der Durchführung

Nicht valider Self-Report, keine Beschreibung der Durchführung

Unklar, welcher Self-Report genutzt wurde

Medication Counts/Weighings $(\mathrm{N}=57)$

Angekündigt

Unangekündigt

Unklar
5

3

49
(9)

\subsection{Eingeschlossene Studien}

1. Adler DA, Bungay KM, Wilson IB, Pei Y, Supran S, Peckham E, Cynn DJ, Rogers WH (2004): The impact of a pharmacist intervention on 6-month outcomes in depressed primary care patients. Gen Hosp Psychiatry 26, 199-209

2. Akerblad AC, Bengtsson F, Ekselius L, von Knorring L (2003): Effects of an educational compliance enhancement programme and therapeutic drug monitoring on treatment adherence in depressed patients managed by general practitioners. Int Clin Psychopharmacol 18, 347-354

3. Al Eidan FA, McElnay JC, Scott MG, McConnell JB (2002): Management of Helicobacter pylori eradication--the influence of structured counselling and follow-up. Br J Clin Pharmacol $\underline{53}$, 163171

4. Altice FL, Mezger JA, Hodges J, Bruce RD, Marinovich A, Walton M, Springer SA, Friedland GH (2004): Developing a directly administered antiretroviral therapy intervention for HIV-infected drug

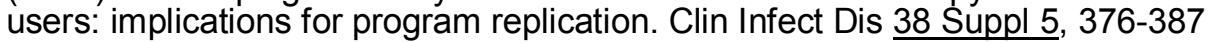

5. Anderson S (1987): Evaluation of pharmacist discharge medication counselling. Aust J Hosp Pharm 17, 131-136

6. Bailey WC, Richards JM, Jr., Brooks CM, Soong SJ, Windsor RA, Manzella BA (1990): A randomized trial to improve self-management practices of adults with asthma. Arch Intern Med $150,1664-1668$

7. Bass MJ, McWhinney IR, Donner A (1986): Do family physicians need medical assistants to detect and manage hypertension? CMAJ 134, 1247-1255

8. Baum D, Creer TL (1986): Medication compliance in children with asthma. J Asthma 23, 49-59

9. Beardsley RS, Johnson CA, Kabat HF (1982): A drug self-administration program: a behavioral approach to patient education. Contemp Pharm Pract $\underline{5}, 156-160$

10. Beck DE, Fennell RS, Yost RL, Robinson JD, Geary D, Richards GA (1980): Evaluation of an educational program on compliance with medication regimens in pediatric patients with renal transplants. J Pediatr $\underline{96}, 1094-1097$

11. Begley S, Livingstone C, Hodges N, Williamson V (1997): Impact of domiciliary pharmacy visits on medication management in an elderly population. Int J Pharm Pract $\underline{5}, 111-121$

12. Berg J, Dunbar-Jacob J, Sereika SM (1997): An evaluation of a self-management program for adults with asthma. Clin Nurs Res $\underline{6}, 225-238$

13. Berrien VM, Salazar JC, Reynolds E, Mckay K (2004): Adherence to Antiretroviral Therapy in HIV-Infected Pediatric Patients Improves with Home-Based Intensive Nursing Intervention. AIDS Patient Care STDS $\underline{18}$, 355-363

14. Birrer RB, Chille E, Weiner M, Gruber S (1983): Hypertensions: A double-blind study of compliance in an urban community. Fam Pract Res $\mathrm{J} \underline{3}, 16$

15. Birtwhistle RV, Godwin MS, Delva MD, Casson RI, Lam M, MacDonald SE, Seguin R, Ruhland L (2004): Randomised equivalence trial comparing three and six months of follow up of patients with hypertension by family practitioners. Br Med J $\underline{328}$, 204-206 
16. Boczkowski JA, Zeichner A, DeSanto N (1985): Neuroleptic compliance among chronic schizophrenic outpatients. J Consult Clin Psychol $\underline{53}, 666-671$

17. Boissel JP, Meillard O, Perrin-Fayolle E, Ducruet T, Alamercery $Y$, Sassano P, Benghozi R (1996): Example of a phase IV trial involving several GPs and aiming at answering a scientific question. Therapie $\underline{51}, 667-676$

18. Brook $\mathrm{OH}$, van Hout $\mathrm{H}$, Stalman W, Nieuwenhuyse H, Bakker B, Heerdink E, de Haan M (2005): A pharmacy-based coaching program to improve adherence to antidepressant treatment among primary care patients. Psychiatr Serv $\underline{56}, 487-489$

19. Burkhart PV, Dunbar-Jacob JM, Fireman P, Rohay J (2002): Children's adherence to recommended asthma self-management. Pediatr Nurs $\underline{28}, 409-414$

20. Casey R, Rosen B, Glowasky A, Ludwig S (1985): An intervention to improve follow-up of patients with otitis media. Clin Pediatr 24, 149-152

21. Chaisson RE, Barnes GL, Hackman J, Watkinson L, Kimbrough L, Metha S, Cavalcante S, Moore RD (2001): A randomized, controlled trial of interventions to improve adherence to isoniazid therapy to prevent tuberculosis in injection drug users. Am J Med 110, 605-610

22. Chisholm MA, Mulloy LL, Jagadeesan M, DiPiro JT (2001): Impact of clinical pharmacy services on renal transplant patients' compliance with immunosuppressive medications. Clin Transplant 15, 330-336

23. Cochran SD (1984): Preventing medical noncompliance in the outpatient treatment of bipolar affective disorders. J Consult Clin Psychol $\underline{52}$, 873-878

24. Cockburn J, Reid AL, Bowman JA, Sanson-Fisher RW (1987): Effects of intervention on antibiotic compliance in patients in general practice. Med J Aust 147, 324-328

25. Crilly M, Esmail A (2005): Randomised controlled trial of a hypothyroid educational booklet to improve thyroxine adherence. Br J Gen Pract $\underline{55}, 362-368$

26. Da Costa FA, Guerreiro JP, Nunes DM, Da Costa MA, Martins AP, Garca o J, Madureira B (2005): Effect of reminder cards on compliance with antihypertensive medication. Int J Pharm Pract $\underline{13}, 205-211$

27. De Tullio PL, Kirking DM, Arslanian C, Olson DE (1987): Compliance measure development and assessment of theophylline therapy in ambulatory patients. J Clin Pharm Ther $\underline{12}, 19-26$

28. Elixhauser A, Eisen SA, Romeis JC, Homan SM (1990): The effects of monitoring and feedback on compliance. Med Care $\underline{28}, 882-893$

29. Ellis DA, Naar-King S, Frey M, Templin T, Rowland M, Greger N (2004): Use of multisystemic therapy to improve regimen adherence among adolescents with type 1 diabetes in poor metabolic control. J Clin Psychol Med Settings 11, 315-324

30. Eshelman FN, Fitzloff $J$ (1976): Effect of packaging on patient compliance with an antihypertensive medication. Curr Ther Res Clin Exp 20, $215-219$

31. Fairley CK, Levy R, Rayner CR, Allardice K, Costello K, Thomas C, McArthur C, Kong D, Mijch A, Melbourne Adherence Group, Melbourne Adherence Group (2003): Randomized trial of an adherence programme for clients with HIV. Int J STD AIDS $\underline{14}$, 805-809

32. Fattal O, Lampe E, Barcelona I, Muzina D (2005): Patient Compliance With Medication and Follow-up Appointments. Psychiatr Ann $\underline{35}, 178$

33. Faulkner MA, Wadibia EC, Lucas BD, Hilleman DE (2000): Impact of pharmacy counseling on compliance and effectiveness of combination lipid-lowering therapy in patients undergoing coronary artery revascularization. Pharmacotherapy $\underline{20}, 410-416$

34. Fennell RS, Foulkes LM, Boggs SR (1994): Family-based program to promote medication compliance in renal transplant children. Transplant Proc $\underline{26}, 102-103$

35. Fiellin DA, Pantalon MV, Chawarski MC, Moore BA, Sullivan LE, O'Connor PG, Schottenfeld RS (2006): Counseling plus buprenorphine-naloxone maintenance therapy for opioid dependence. $\mathrm{N}$ Engl J Med 355, 365-374

36. Gabriel M, Gagnon JP, Bryan CK (1977): Improved patients compliance through use of a daily drug reminder chart. Am J Public Health $\underline{67}, 968-969$

37. Gani F, Pozzi E, Crivellaro MA, Senna G, Landi M, Lombardi C, Canonica GW, Passalacqua G (2001): The role of patient training in the management of seasonal rhinitis and asthma: clinical implications. Allergy $\underline{56}, 65-68$

38. Garety PA, Craig TK, Dunn G, Fornells-Ambrojo M, Colbert S, Rahaman N, Read J, Power P (2006): Specialised care for early psychosis: symptoms, social functioning and patient satisfaction: randomised controlled trial. Br J Psychiatry 188, 37-45 
39. Gillis LS, Koch A, Joyl M (1989): Improving compliance in Xhosa psychiatric patients. S Afr Med J 76, 205-208

40. Ginde AA, Von Harz BC, Turnbow D, Lewis LM (2003): The effect of ED prescription dispensing on patient compliance. Am J Emerg Med 21, 313-315

41. Girvin B, McDermott BJ, Johnston GD (1999): A comparison of enalapril $20 \mathrm{mg}$ once daily versus $10 \mathrm{mg}$ twice daily in terms of blood pressure lowering and patient compliance. J Hypertens 17 , 1627-1631

42. Golin CE, Earp J, Tien HC, Stewart P, Porter C, Howie L (2006): A 2-arm, randomized, controlled trial of a motivational interviewing-based intervention to improve adherence to antiretroviral therapy (ART) among patients failing or initiating ART. J Acquir Immune Defic Syndr 42, 42-51

43. Gonzalez-Fernandez RA, Rivera M, Torres D, Quiles J, Jackson A (1990): Usefulness of a systemic hypertension in-hospital educational program. Am J Cardiol $\underline{65}, 1384-1386$

44. Graham MR, Lindsey CC, Kennedy JA (2002): Maintenance of low-density lipoprotein goal with step-down pravastatin therapy. Pharmacotherapy 22, 21-26

45. Gupta U, Sharma S, Sheth PD, Jha J, Chaudhury RR (2005): Improving medicine usage through patient information leaflets in India. Trop Doct $\underline{35}$, 164-166

46. Guthrie RM (2001): The effects of postal and telephone reminders on compliance with pravastatin therapy in a national registry: results of the first myocardial infarction risk reduction program. Clin Ther $\underline{23}, 970-980$

47. Hamilton GA (2003): Measuring adherence in a hypertension clinical trial. Eur J Cardiovasc Nurs $\underline{2}, 219-228$

48. Hamilton GA, Roberts SJ, Johnson JM, Tropp JR, Anthony-Odgren D, Johnson BF (1993): Increasing adherence in patients with primary hypertension. Health Values 17 , 3-11

49. Hecht AB (1974): Improving medication compliance by teaching outpatients. Nurs Forum 13 , $112-$ 129

50. Hederos C-A, Janson S, Hedlin G (2005): Group discussions with parents have long-term positive effects on the management of asthma with good cost-benefit. Acta Paediatr $\underline{94}, 602-608$

51. Henry A, Batey RG (1999): Enhancing compliance not a prerequisite for effective eradication of Helicobacter pylori: the HelP Study. Am J Gastroenterol 94, 811-815

52. Holzemer WL, Bakken S, Portillo CJ, Grimes R, Welch J, Wantland D, Mullan JT (2006): Testing a nurse-tailored HIV medication adherence intervention. Nurs Res $\underline{55}, 189-197$

53. Hovell MF, Sipan CL, Blumberg EJ, Hofstetter CR, Slymen D, Friedman L, Moser K, Kelley NJ, Vera AY (2003): Increasing Latino adolescents' adherence to treatment for latent tuberculosis infection. Am J Public Health $\underline{93}$, 1871-1877

54. Johnson AL, Taylor DW, Sackett DL, Dunnett CW, Shimizu AG (1978): Self-recording of blood pressure in the management of hypertension. Can Med Assoc J 119, 1034-1039

55. Katon W, Robinson P, Von Korff M, Lin E, Bush T, Ludman E, Simon G, Walker E (1996): A multifaceted intervention to improve treatment of depression in primary care. Arch Gen Psychiatry $\underline{53}, 924-932$

56. Katon W, Von Korff M, Lin E, Simon G, Walker E, Unutzer J, Bush T, Russo J, Ludman E (1999): Stepped collaborative care for primary care patients with persistent symptoms of depression. Arch Gen Psychiatry $\underline{56}, 1109-1115$

57. Katon W, Rutter C, Ludman EJ, Von Korff M, Lin E, Simon G, Bush T, Walker E, Unutzer J (2001): A randomized trial of relapse prevention of depression in primary care. Arch Gen Psychiatry $\underline{58}, 241-247$

58. Kirscht JP, Kirscht JL, Rosenstock IM (1981): A test of interventions to increase adherence to hypertensive medical regimens. Health Educ Q $\underline{8}, 261-272$

59. Kozuki Y, Schepp KG (2006): Visual-feedback therapy for antipsychotic medication adherence. Int Clin Psychopharmacol 21, 57-61

60. Laporte S, Quenet S, Buchmuller-Cordier A, Reynaud J, Tardy-Poncet B, Thirion C, Decousus H, Mismetti P (2003): Compliance and stability of INR of two oral anticoagulants with different halflives. Thromb Haemost $\underline{89}$, 458-467

61. LeBaron S, Zeltzer LK, Ratner P, Kniker WT (1985): A controlled study of education for improving compliance with cromolyn sodium (Intal): the importance of physician-patient communication. Ann Allergy $\underline{55}, 811-808$ 
62. Levine AM, Richardson JL, Marks G, Chan K, Graham J, Selser JN, Kishbaugh C, Shelton DR, Johnson CA (1987): Compliance with oral drug therapy in patients with hematologic malignancy. $\mathrm{J}$ Clin Oncol $\underline{5}, 1469-1476$

63. Logan AG, Milne BJ, Achber C, Campbell WP, Haynes RB (1979): Work-site treatment of hypertension by specially trained nurses. Lancet $1979,2,1175-1178$

64. Ludman E, Katon W, Bush T, Rutter C, Lin E, Simon G, Von Korff M, Walker E (2003): Behavioural factors associated with symptom outcomes in a primary care-based depression prevention intervention trial. Psychol Med $\underline{33}$, 1061-1070

65. Maiman LA, Becker MH, Liptak GS, Nazarian LF, Rounds KA (1988): Improving pediatricians' compliance-enhancing practices. Am J Dis Child 142, 773-779

66. Manders AJ, Banerjee A, van den Borne HW, Harries AD, Kok GJ, Salaniponi FM (2001): Can guardians supervise TB treatment as well as health workers? A study on adherence during the intensive phase. Int J Tuberc Lung Dis $\underline{5}, 838-842$

67. Mansoor LE, Dowse R (2006): Medicines information and adherence in HIVIAIDS patients. J Clin Pharm Ther $\underline{31}, 7-15$

68. Matsuyama J, Mason B, Jue S (1993): Pharmacists' interventions using an electronic medicationevent monitoring device's adherence data versus pill counts. Ann Pharmacother 27, 851-855

69. Miklowitz DJ, George EL, Richards JA, Simoneau TL, Suddath RL (2003): A randomized study of family-focused psychoeducation and pharmacotherapy in the outpatient management of bipolar disorder. Arch Gen Psychiatry $\underline{60}$, 904-912

70. Morisky DE, Malotte CK, Choi P, Davidson P, Rigler S, Sugland B, Langer M (1990): A patient education program to improve adherence rates with antituberculosis drug regimens. Health Educ Q 17, 253-267

71. Myers ED, Branthwaite A (1992): Out-patient compliance with antidepressant medication. Br J Psychiatry 160, 83-86

72. Myers ED, Calvert EJ (1984): Information, compliance and side-effects: a study of patients on antidepressant medication. Br J Clin Pharmacol $\underline{17}, 21-25$

73. Nessman DG, Carnahan JE, Nugent CA (1980): Increasing compliance. Patient-operated hypertension groups. Arch Intern Med 140, 1427-1430

74. Nides MA, Tashkin DP, Simmons MS, Wise RA, Li VC, Rand CS (1993): Improving inhaler adherence in a clinical trial through the use of the nebulizer chronolog. Chest 104, 501-507

75. Nunes EV, Rothenberg JL, Sullivan MA, Carpenter KM, Kleber HD (2006): Behavioral therapy to augment oral naltrexone for opioid dependence: a ceiling on effectiveness? Am J Drug Alcohol Abuse 32, 503-517

76. Okonkwo PO, Akpala CO, Okafor HU, Mbah AU, Nwaiwu O (2001): Compliance to correct dose of chloroquine in uncomplicated malaria correlates with improvement in the condition of rural $\mathrm{Ni}$ gerian children. Trans R Soc Trop Med Hyg $\underline{95}$, 320-314

77. Peterson GM, McLean S, Millingen KS (1984): A randomised trial of strategies to improve patient compliance with anticonvulsant therapy. Epilepsia 25, 407-412

78. Pettinati HM, Volpicelli JR, Pierce JD, Jr., O'Brien CP (2000): Improving naltrexone response: an intervention for medical practitioners to enhance medication compliance in alcohol dependent patients. J Addict Dis $\underline{19}, 71-83$

79. Peveler R, George C, Kinmonth AL, Campbell M, Thompson C (1999): Effect of antidepressant drug counselling and information leaflets on adherence to drug treatment in primary care: randomised controlled trial. BMJ $\underline{319}$, 612-615

80. Ponnusankar S, Surulivelrajan M, Anandamoorthy N, Suresh B (2004): Assessment of impact of medication counseling on patients' medication knowledge and compliance in an outpatient clinic in South India. Patient Educ Couns 54, 55-60

81. Purcell DW, Metsch LR, Latka M, Santibanez S, Gomez CA, Eldred L, Latkin CA, INSPIRE Study Group (2004): Interventions for seropositive injectors-research and evaluation: an integrated behavioral intervention with HIV-positive injection drug users to address medical care, adherence, and risk reduction. J Acquir Immune Defic Syndr 37 Suppl 2, 108-110

82. Putnam DE, Finney JW, Barkley PL, Bonner MJ (1994): Enhancing commitment improves adherence to a medical regimen. J Consult Clin Psychol $\underline{62}, 191-194$

83. Rathbun RC, Farmer KC, Stephens JR, Lockhart SM (2005): Impact of an adherence clinic on behavioral outcomes and virologic response in treatment of HIV infection. Clin Ther 27, 199-209 
84. Rehder TL, McCoy LK, Blackwell B, Whitehead W, Robinson A (1980): Improving medication compliance by counseling and special prescription container. Am J Hosp Pharm $\underline{37}$, 379-385

85. Reid SC, Teesson M, Sannibale C, Matsuda M, Haber PS (2005): The efficacy of compliance therapy in pharmacotherapy for alcohol dependence. J Stud Alcohol $\underline{66}, 833-841$

86. Revankar CR, Gupta N, Sorensen BH, Naik SS, Sharma BL, Mangalani PR, Gupta SL, Mishra GP, Gude SL, Swamy A, Satpathy SN, Patnaik SB, Swaminathan TN, Pandian TD (1993): Further observations on MDT blister-calendar packs in vertical leprosy eradication programmes. Lepr $\operatorname{Rev} \underline{64}, 250-254$

87. Richardson JL, Marks G, Johnson CA, Graham JW, Chan KK, Selser JN, Kishbaugh C, Barranday Y, Levine AM (1987): Path model of multidimensional compliance with cancer therapy. Health Psychol $\underline{6}, 183-207$

88. Rickles NM, Svarstad BL, Statz-Paynter JL, Taylor LV, Kobak KA (2005): Pharmacist telemonitoring of antidepressant use: effects on pharmacist-patient collaboration. J Am Pharm Assoc 45, 344-353

89. Rosen MI, Rigsby MO, Salahi JT, Ryan CE, Cramer JA (2004): Electronic monitoring and counseling to improve medication adherence. Behav Res Ther $\underline{42}$, 409-422

90. Rossini M (2000): Effects of two intermittent alendronate regimens in the prevention or treatment of postmenopausal osteoporosis. Bone 27, 119-122

91. Samet JH, Horton NJ, Meli S, Dukes K, Tripps T, Sullivan L, Freedberg KA (2005): A randomized controlled trial to enhance antiretroviral therapy adherence in patients with a history of alcohol problems. Antivir Ther 10, 83-93

92. Schectman G, Hiatt J, Hartz A (1994): Telephone contacts do not improve adherence to niacin or bile acid sequestrant therapy. Ann Pharmacother $\underline{28}, 29-35$

93. Schwartz RH, Wientzen RL, Jr., Pedreira F, Feroli EJ, Mella GW, Guandolo VL (1981): Penicillin $\checkmark$ for group $A$ streptococcal pharyngotonsillitis. A randomized trial of seven vs ten days' therapy. JAMA 246, 1790-1795

94. Seltzer A, Roncari I, Garfinkel P (1980): Effect of patient education on medication compliance. Can J Psychiatry 25, 638-645

95. Stein MD, Herman DS, Solomon DA, Anthony JL, Anderson BJ, Ramsey SE, Miller IW (2004): Adherence to treatment of depression in active injection drug users: the minerva study. J Subst Abuse Treat 26, 87-93

96. Strang JS, Falloon IRH, Moss HB (1981): The effects of family therapy on treatment compliance in schizophrenia. Psychopharmacol Bull 17, 87-88

97. Streicker SK, Amdur M, Dincin J (1986): Educating patients about psychiatric medications: failure to enhance compliance. Psychiatr Rehabil J $\underline{9}, 15-28$

98. Sturgess IK, McElnay JC, Hughes CM, Crealey G (2003): Community pharmacy based provision of pharmaceutical care to older patients. Pharm World Sci 느, 218-226

99. Swartz MS, Swanson JW, Wagner HR, Burns BJ, Hiday VA (2001): Effects of involuntary outpatient commitment and depot antipsychotics on treatment adherence in persons with severe mental illness. J Nerv Ment Dis $\underline{189}$, 583-592

100. Taggart AJ, Johnston GD, McDevitt DG (1981): Does the frequency of daily dosage influence compliance with digoxin therapy? Br J Clin Pharmacol 11, 31-34

101. Tarpay M, Marks MI, Hopkins C (1982): Cefaclor therapy twice daily for acute otitis media. Controlled comparative study of twice-daily vs thrice-daily dose schedule. Am J Dis Child $\underline{136}, 33-$ 35

102. Tierney WM, Overhage JM, Murray MD, Harris LE, Zhou XH, Eckert GJ, Smith FE, Nienaber N, McDonald CJ, Wolinsky FD (2003): Effects of computerized guidelines for managing heart disease in primary care. J Gen Intern Med 18, 967

103. Tuldra A, Fumaz CR, Ferrer MJ, Bayes R, Arno A, Balague M, Bonjoch A, Jou A, Negredo E, Paredes R, Ruiz L, Romeu J, Sirera G, Tural C, Burger D, Clotet B (2000): Prospective randomized two-Arm controlled study to determine the efficacy of a specific intervention to improve longterm adherence to highly active antiretroviral therapy. J Acquir Immune Defic Syndr $\underline{25}, 221-228$

104. Varma S, McElnay JC, Hughes CM, Passmore AP, Varma M (1999): Pharmaceutical care of patients with congestive heart failure: interventions and outcomes. Pharmacotherapy $\underline{19}, 860-869$

105. Visnegarwala F, Rodriguez-Barradass MC, Graviss EA, Caprio M, Nykyforchyn M, Laufman L (2006): Community outreach with weekly delivery of anti-retroviral drugs compared to cognitivebehavioural health care team-based approach to improve adherence among indigent women newly starting HAART. AIDS Care $\underline{18}, 332-338$ 
106. Von Korff M, Katon W, Rutter C, Ludman E, Simon G, Lin E, Bush T (2003): Effect on disability outcomes of a depression relapse prevention program. Psychosom Med $\underline{65}$, 938-943

107. Wall TL, Sorensen JL, Batki SL, Delucchi KL, London JA, Chesney MA (1995): Adherence to zidovudine (AZT) among HIV-infected methadone patients: a pilot study of supervised therapy and dispensing compared to usual care. Drug Alcohol Depend 37, 261-269

108. Weber R, Christen L, Christen S, Tschopp S, Znoj H, Schneider C, Schmitt J, Opravil M, Gunthard HF, Ledergerber B, Swiss HIV CS (2004): Effect of individual cognitive behaviour intervention on adherence to antiretroviral therapy. Antivir Ther $\underline{9}, 85-95$

109. Williams RL, Maiman LA, Broadbent DN, Kotok D, Lawrence RA, Longfield LA, Mangold AH, Mayer SJ, Powell KR, Sayre JW (1986): Educational strategies to improve compliance with an antibiotic regimen. Am J Dis Child 140, 216-220

110. Windsor RA, Bailey WC, Richards JM, Jr., Manzella B, Soong SJ, Brooks M (1990): Evaluation of the efficacy and cost effectiveness of health education methods to increase medication adherence among adults with asthma. Am J Public Health $\underline{80}$, 1519-1521

111. Winland-Brown JE, Valiante J (2000): Effectiveness of different medication management approaches on elders' medication adherence. Outcomes Manag Nurs Pract $\underline{4}, 172-176$

112. Zhang M, Wang M, Li J, Phillips MR (1994): Randomised-control trial of family intervention for 78 first-episode male schizophrenic patients. Br J Psychiatry Suppl 165, 24-102 


\section{Diskussion}

\subsection{Wichtigste Ergebnisse}

In unserem Review konnten wir feststellen, dass selbst in adhärenzverbessernden Interventionsstudien die Adhärenz oft unzuverlässig und nicht entsprechend den einschlägigen Empfehlungen gemessen wird. Die Anzahl der in einer Studie angewandten Messmethoden ist häufig unzureichend. Die uneinheitliche Durchführung der Messungen sowie die ungenügende Erläuterung der Begriffe bzw. der Ergebnisse sind weitere Mängel.

Von den 448 eingeschlossenen Studien nutzten 336 nur eine Messmethode, 35 dieser Studien zudem eine unvalide Messmethode; 112 Studien verwendeten mehr als eine Messmethode, doch nur 24 Studien erfüllten die vorab definierten Qualitätskriterien (fast) vollständig. Auf die 448 Studien umgerechnet, sind somit nur ca. $5 \%$ der Studien als qualitativ gut einzuschätzen.

Dies spiegelt sich auch in der speziellen Adhärenzbewertung der am häufigsten verwendeten Messverfahren (Self-Report und Medication Count/Weighing) wider. Die Beschreibung war bei beiden Methoden überwiegend mangelhaft und entscheidende Details zur Einschätzung der Messungen fehlten. Bei den Self-Reports wurden nur in wenigen Fällen valide Formen genutzt.

\subsection{Stärken und Schwächen der Arbeit}

Eine systematische Übersichtsarbeit sollte mit größtmöglicher Sorgfalt in Hinblick auf die Methodik durchgeführt werden. Wir orientierten uns hierfür an den Empfehlungen der Cochrane Collaboration zur Erstellung systematischer Übersichtsarbeiten (Higgins et al. 2006). Ziele, Studienkriterien und Vorgehen bei der Literatursuche wurden in einem a-priori-Protokoll festgehalten.

Wir haben unseren Review auf (randomisiert) kontrollierte Studien (RCT) fokussiert, da sie als Goldstandard für die klinische Forschung angesehen werden (Phillips et al. 2001). Des Weiteren ist davon auszugehen, dass das Studiendesign auch Einfluss auf weitere qualitative Aspekte der Studie hat (Colditz et al. 1989). 
Die Literaturrecherche ist für einen Systematic Review von besonderer Bedeutung. Es wurde eine Suchstrategie erstellt, die wir mit Suchstrategien anderer Studien (Haynes et al. 2005, Simpson et al. 2006) verglichen. Nach diversen Probesuchen, in denen wir überprüft haben, ob die Suche relevante Studien aus anderen Systematic Reviews über adhärenzverbessernde Interventionen findet, haben wir die Suchstrategie einer kanadischen Forschungsgruppe, die im Jahr 2006 eine Metaanalyse zum Thema „Mortalität und Adhärenz“ angefertigt hat (Simpson et al. 2006), vorgelegt und gemäß den Empfehlungen weiter modifiziert. Um mögliche Verzerrungen durch einen „RCT-Filter“ zu vermeiden, haben wir nach allen Publikationstypen gesucht. Es ist bekannt, dass Systematic Reviews sehr anfällig für den Publication Bias sind. Das bedeutet, dass Studien mit nicht signifikantem Ergebnis später oder gar nicht veröffentlicht werden. Bei einer Veröffentlichung werden diese Studien dann häufiger in nicht-englischsprachigen Zeitschriften publiziert. Um diese Verzerrung zu minimieren, haben wir die Literatursuche nicht auf eine bestimmte Sprache beschränkt. Außerdem wurden neben MedLine (hier sind vor allem angloamerikanische Zeitschriften gelistet) weitere Datenbanken wie Embase (1980 bis November 2002), Cochrane Controlled Trials Register (1970 bis November 2002), CINAHL (1982 bis Februar 2006) und PsycINFO durchsucht. Anschließend an die elektronische Suche haben wir noch eine ausführliche „Hand-Search“ in den Referenzen der eingeschlossenen Studien und relevanten Systematic Reviews durchgeführt, um eventuell fehlende Artikel zu finden.

Bei der Abstract-/Volltextauswertung können Fehler entstanden sein, indem relevante Artikel trotz Erfüllung der Einschlusskriterien ausgeschlossen wurden. Dieses Risiko wurde reduziert, da die Artikel von zwei Personen unabhängig voneinander ausgewertet wurden.

Die eingeschlossenen Studien wurden nach den in Kapitel 4.3 (S. 20-24) genannten Variablen kodiert und zusätzlich zu dieser Kodierung folgte eine Freitextanlage, um auch schwer zu kategorisierende Variablen festzuhalten. Diese breit angelegte Analyse gibt dadurch einen umfassenden Einblick in die Adhärenzmessung (in Interventionsstudien). 
Bei der Erstellung unseres Reviews wurden hohe Studieneinschlusskriterien (nur kontrollierte, adhärenzverbessernde Interventionsstudien, Volltextauswertung nur bei Studien mit mehr als zwei Messmethoden) verwendet. Diese hohe Filterung könnte eine Schwäche des Reviews sein, da hierdurch eine Verzerrung entstehen kann, die den Stand der Adhärenzmessung ungenau abbildet und nur sehr spezifisch zu verwerten ist. Wir sind aber der Meinung, dass die gewonnenen Hauptergebnisse aus unserem Review durch die Filterkriterien ihre Aussagekraft nicht verlieren. Hätten wir die Einschlusskriterien offener formuliert und „schlechter angelegte“ Studien (mit z.B. nur einer Messmethode) bei der Volltextauswertung eingeschlossen, wäre das negative Ergebnis über die Qualität der Adhärenzmessung wohl noch deutlicher ausgefallen. Pharmakologische Studien müssen aufgrund ihrer Bedeutung mittlerweile hohe Standards erfüllen. Bei der begleitenden und für die Interpretation der Studien wichtigen Adhärenzmessung sind dagegen keine Standards vorhanden. Falls man sich entscheidet, Adhärenz zu messen, sollte dies nach den bisher gegebenen Empfehlungen geschehen, da Adhärenzmessung sonst als Alibimessung angesehen werden kann. Für adhärenzverbessernde Interventionsstudien, die Adhärenz sogar als primäres Outcome messen, sollten mindestens die gleichen Ansprüche gelten. Auch aus diesem Grund haben wir uns für diese strengen Einschlusskriterien entschieden.

Eine Stärke und gleichzeitige Schwäche für unsere Übersichtsarbeit ist die Inhomogenität der gefundenen Studien. Es wurden verschiedene Erkrankungen, Interventionsansätze und Messmethoden untersucht. Diese Inhomogenität führt dazu, dass spezifische Fragestellungen zu den Erkrankungen, den einzelnen Interventionen und Messmethoden nur eingeschränkt möglich waren. Dieser breite Ansatz liefert andererseits einen allgemeinen Überblick der Adhärenzmessung und bietet Einblick in die wichtigsten Aspekte der Adhärenzmessung.

\subsection{Literaturvergleich}

Auch wenn eine große Anzahl von systematischen Übersichtsarbeiten zum Thema Adhärenz existiert, ist dies nach unserem Kenntnistand die erste Arbeit, die sich in diesem Umfang mit Verwendung, Durchführung und Qualität der Adhärenzmessung beschäftigt. 
Die am ehesten vergleichbare Studie ist von Gossec et al. (2007) und befasst sich mit Adhärenzmessung bei 6 chronischen Erkrankungen: HIV, Diabetes mellitus, rheumatoide Arthritis, Asthma, arterieller Hypertonus und Osteoporose. Sechs HighImpact-Journals der Allgemeinmedizin und Inneren Medizin sowie die zu den jeweiligen Erkrankungen zugehörigen High-Impact-Journals wurden nach randomisiert kontrollierten Studien durchsucht. Es wurden nur englischsprachige pharmakologische Studien aus dem Jahr 2003 und 2004 eingeschlossen, bei denen die Patienten Medikamente eigenständig einnahmen. Die Autoren bezeichnen die Arbeit selber als eine Pilotstudie, mit dem Ziel herauszufinden, welche Messmethoden verwendet wurden und wie Adhärenz gemessen wird. Neben den grundlegenden methodischen Aspekten (Studienlänge, Art der Förderung, Grunderkrankung u.a.), wurde die Art der Messmethode und im Besonderen die Ergebnispräsentation untersucht. Von 192 eingeschlossenen Studien wurde Adhärenz in 69 (36 \%) gemessen. Von diesen 69 Studien, die Adhärenz gemessen hatten, nutzten 18 (26 \%) zwei oder mehrere Messmethoden. Eine getrennte Ergebnisdarstellung gab es dabei in nur zwei Studien. In 21 Studien fehlten die Ergebnisse der Adhärenzmessung, in 26 Studien die Beschreibung der Adhärenzmessung. Adhärenz wurde v.a. quantitativ gemessen (41 vs. 27 Studien). Studien, die Cut-offs verwendeten, zeigten starke Variationen in diesen. Die am häufigsten verwendeten Messmethoden waren Medication Count/Weighing und Self Report. HIV und Diabetes mellitus waren die häufigsten Grunderkrankungen.

Aufgrund der z.T. unterschiedlichen Fragestellung und den verschiedenen Einschlusskriterien ist diese Arbeit mit unserem Review nur in gewissem Maße vergleichbar. Insgesamt untersucht unser Systematic Review mit den Interventionsstudien eine andere Art von Studien und ist dabei durch Berücksichtigung mehrere Erkrankungen und einer breit durchgeführten Literatursuche umfassender. Trotzdem existieren einige Parallelen. Beide Reviews kamen zu dem Ergebnis, dass nur in ca. $25 \%$ der untersuchten Studien mehr als eine Messmethode genutzt wurde. Die Ergebnisdarstellung bzw. die Beschreibung bewerteten Gossec et al. (2007) häufig als mangelhaft. Das entsprach auch unserer Bewertung. Adhärenz wurde - so das übereinstimmende Ergebnis beider Reviews - in den meisten Studien quantitativ und v.a. mit Self Report und Medication Count/Weighing gemessen. Verwendete Cut-offs waren sehr unterschiedlich definiert und variierten. Die Grunderkrankungsverteilung 
ist aufgrund der eingrenzenden Einschlusskriterien des Reviews von Gossec et al. (2007) nicht mit unserem Review zu vergleichen.

Eine weitere, zumindest ähnliche Arbeit stammt von Nichol et al. (1999). In dieser Metaanalyse wurde auch die Qualität von Adhärenzstudien bestimmt. Die qualitative Auswertung bezog sich auf Studiendesign, Krankheitsspezifikation und Adhärenzmessung/-definition. 72 Studien waren in diese Metaanalyse eingeschlossen und das Hauptergebnis entspricht unserer Arbeit. In beiden Fällen wird wegen der Adhärenzmessung/-definition sowie des Studiendesigns und der Krankheitsspezifikation die Gesamtqualität der Studien deutlich abgewertet. So beschrieben Nichol et al. (1999), dass die Adhärenzdefinition nur bei gut $40 \%$ nachvollziehbar war, bei ca. 30 \% der Studien fanden sie gar keine Adhärenzdefinition. Etwa 30 \% der Studien verzichteten nach Nichol et al. (1999) auf die Angabe des Adhärenzmessinstruments oder es wurden nicht validierte Messinstrumente genutzt. Über die Hälfte der Studien kam aus Amerika, was in etwa unserem Ergebnis entspricht.

Auch hier ist die Vergleichbarkeit zu unserem Review jedoch nur eingeschränkt möglich, da es einige methodische Unterschiede gibt. Die Literatursuche war z.B. auf MedLine und englischsprachige Artikel begrenzt. Es wurden sämtliche Artikel, die sich mit Medikamentenadhärenz auseinandersetzten, eingeschlossen - unabhängig vom methodischen Design (fast zwei Drittel der Studien waren deskriptiv und nur ca. $10 \%$ wiesen ein Prä-/Post-Test Design auf) bzw. vom Studientyp (eingeschlossen waren z.B. Interventionsstudien zur Adhärenzverbesserung als auch Studien, in denen Adhärenz sekundär gemessen wurde). Messmethoden wurden im Gegensatz zu unserer Arbeit gewichtet (so wurde z.B. ein validierter Self-Report mit einem Punkt und eine unangekündigte Laborbestimmung in Kombination mit einem Medication Count/Weighing mit 6 Punkten bewertet). Diese Bewertung ist nur eingeschränkt nachvollziehbar. Die Verteilung der Grunderkrankung unterschied sich ebenso von unseren Ergebnissen. In ca. einem Viertel der Fälle (20/77) wurden kardiovaskuläre Krankheiten gefolgt von infektiösen (15\%), respiratorischen (14\%) und psychiatrischen (11\%) Erkrankungen untersucht.

Eine große Metaanalyse zu Adhärenz von DiMatteo et al. (2002) hat sich in einem Nebenaspekt mit der Anzahl der Messmethoden beschäftigt. Hierbei wurde festgestellt, dass es in den letzten Jahren eher zu einer Abnahme der Messmethodenzahl 
gekommen ist. Wir können diesen Trend in unserer Arbeit nicht bestätigen - die mittlere Messmethodenanzahl zeigt nach unserer Auswertung in den letzten 5 Jahren im Vergleich zu den letzten 20 Jahren eher einen leichten Anstieg. Auch im Vergleich zu DiMatteo (2002) ist ein anderer methodischer Aufbau zu berücksichtigen. In seiner Metaanalyse waren im Gegensatz zu unserem Review z.B. auch nichtmedikamentöse Behandlungen eingeschlossen und psychiatrische Erkrankungen ausgeschlossen.

In einer deskriptiven Übersichtsarbeit von Paterson und Britten (2005) wird die überwiegende Verwendung von unvalidierten Self-Reports zur Adhärenzmessung beschrieben und die Verwendung von validierten Messmethoden gefordert. Es gibt in dieser Arbeit keine Zahlen zur Häufigkeitsverteilung von unvalidierten zu validierten Self-Reports, doch deckt sich die Hauptaussage mit unseren Erkenntnissen.

Zur Verteilung von angekündigten, unangekündigten und unklaren Medication Counts/Weighings haben wir keine Arbeit finden können.

\subsection{Diskussion der Ergebnisse}

Es gibt bereits verschiedene Empfehlungen zur Qualitätssicherung der Adhärenzmessung (Chesney 2006, DiMatteo und Haskard 2006, Peterson et al. 2007):

- Verwendung mehrerer Messmethoden

- Erfassen von objektivierbaren Krankheitsoutcomes

- Beschreibung der verwendeten Adhärenzdefinition

- $\quad$ Präzise Trennung von Adhärenz und Persistenz

- $\quad$ Beachtung spezifischer Empfehlungen für die einzelnen Messmethoden (z.B. Medication Count/Weighing bei EDM, EDM mit Self-Report koppeln).

Unser Review zeigt nun große Schwächen in der Umsetzung dieser Empfehlungen. Die Messungen werden sehr uneinheitlich durchgeführt. Selbst bei Verwendung der gleichen Messmethoden ist die Durchführung der Messung unterschiedlich. Damit sind Vergleiche zwischen den Ergebnissen oder Aussagen über die Effizienz der Interventionen aufgrund der Ergebnisinkonsistenz problematisch. So halten wir es 
z.B. für möglich, dass die unklaren Ergebnisse bezüglich der Interventionswirksamkeit in den systematischen Übersichtsarbeiten von Gorenoi et al. (2007) und Haynes et al. (2005) durchaus in den Schwächen der Adhärenzmessung in den einzelnen Studien begründet sein könnten, wie auch umgekehrt „gute“ Ergebnisse nicht unbedingt auf die Güte der Intervention schließen lassen.

Ein Teil der Probleme scheint durch die schwierige Konzeptualisierung von Adhärenz und die verwirrenden Begrifflichkeiten zu entstehen. Medizinischen Aspekten (Adhärenz als Maßstab der Einhaltung der Therapie) stehen sozialwissenschaftliche Ansätze gegenüber (Non-Adhärenz als Selbstbestimmungsrecht des Patienten). Die Verantwortlichkeit für Non-Adhärenz allein auf den Patienten zurückzuführen, wäre im letzteren Fall nicht akzeptabel. In der Literatur wird empfohlen, anstelle des Begriffs Compliance den moderneren Begriff von Adhärenz zu verwenden, um auch die Verantwortlichkeit des Arztes zu betonen. Da es in den untersuchten Studien jedoch um die „reine“ Messung der Medikamenteneinnahme geht, könnte die Betonung der Arztverantwortung verwirrend sein und der „alte“ Begriff Compliance scheint angemessener.

Nur ein Drittel der Studien nutzte mehr als zwei Messmethoden. Der Begriff „Adhärenz" wurde in den Studien häufig nicht näher ausgeführt und verschiedene Begriffe wie Medikamentenadhärenz und Terminadhärenz wurden miteinander vermengt. In 74 von 112 Studien wurden keine Cut-off-Werte zu Adhärenz verwendet; in den restlichen 38 Studien variierten die Werte stark. In einem Drittel der Studien wurden keine objektivierbaren Krankheitsoutcomes gemessen.

Gerade bei Personen über 65 Jahren und in langdauernden Studien (über 180 Tage Studiendauer) war die Anzahl von Messmethoden geringer, obwohl gerade hier die Adhärenzmessung besonders schwierig, aber gleichzeitig auch besonders wichtig ist. Auch kurzdauernde Studien (bis 20 Tage Studiendauer) nutzten weniger Messmethoden. Des Weiteren wurden je nach Studiendauer unterschiedliche Messmethoden verwendet. Dabei variierten Häufigkeit und Zeitpunkt der Messungen auch bei gleicher Messmethode stark zwischen den Studien. 
Die Anzahl der Messmethoden stand auch im Zusammenhang mit der Einschlussdiagnose. Studien, die sich mit psychischen Erkrankungen beschäftigten, nutzten die höchste Anzahl von Messmethoden. Eine mögliche Erklärung hierfür könnte sein, dass die Adhärenzmessung bei psychischen Krankheiten z.B. durch die veränderte Wahrnehmung oder die Beeinträchtigung der kognitiven Funktionen der Patienten erschwert ist und deswegen mehr Messmethoden verwendet wurden. Studien, die sich mit HIV beschäftigen, nutzten durchschnittlich 2,5 Messverfahren - eine Anzahl, die im Durchschnitt aller hier untersuchten Studien liegt. Bei HIV ist eine hohe Adhärenz sehr wichtig, um die Viruslast zu senken und um die Entstehung von resistenten HIV-Stämmen zu vermeiden (Bartlett 2002). Gleichzeitig erschweren komplexe Regimes und Stigmatisierungsängste das Adhärenzverhalten und es bestehen besondere Herausforderungen an die Adhärenzmessung. Eine Erklärung für die mittlere Messmethodenanzahl könnte die überdurchschnittlich häufige Verwendung von EDM sein. Diese Messmethode wird oft (nicht unbedingt zutreffend) als Goldstandard angesehen. Evtl. haben einige Forschungsgruppen aufgrund des Einsatzes dieses ressourcenfordernden, aber sehr präzisen Instruments die Nutzung weiterer Messmethoden für weniger notwendig angesehen. Ein anderer Grund könnte auch sein, dass die Adhärenzprobleme bei HIV einerseits sehr stark sind, jedoch andererseits die Adhärenzmotivation aufgrund der Lebensnotwendigkeit der Medikamenteneinnahme gegenüber anderen Erkrankungen erhöht ist. DiMatteo hat 2004 z.B. in einem großen Systematic Review die höchsten durchschnittlichen Adhärenzraten bei HIV gefunden (88\%). Somit sind die Anforderungen an die Adhärenzmessung bei HIV evtl. nicht höher einzuschätzen als bei anderen Krankheitsbildern.

Ein weiterer Faktor für die (mangelnde) Anzahl und Qualität der Messmethoden ist das Land, in welchem die Studie durchgeführt wurde. Amerikanische Studien verwendeten im Durchschnitt mehr Messmethoden und dabei besonders häufig Medical/Refill Records. Hierfür könnten strukturelle Unterschiede der Gesundheitssysteme verantwortlich sein. In Amerika werden z.B. viele medizinische Veteraneneinrichtungen genutzt, um medizinische Daten (u.a. Adhärenz der Patienten) zu sammeln. In Deutschland sind solche Apothekenlisten aufgrund des Datenschutzgesetzes verboten. Im amerikanischen Gesundheitssystem spielen außerdem Qualitäts- und Kostenkontrolle eine große Rolle. In den 90er Jahren wurden durch die Health Maintenance Organisations (HMOs) verschiedene Beschlüsse erlassen, die u.a. Qualitäts- 
standards sowie medizinisches und finanzielles Controlling vorgeben. Damit wurde das Ranking und Benchmarking der USA in den medizinischen Bereichen weiter verstärkt. Trotzdem haben die USA eines der teuersten Gesundheitssysteme der Welt (OECD 2008) bei einer nur mittleren Qualität (WHO 2000). Durch Verbesserung der Adhärenz sind weitere Einsparungen möglich und evtl. erklären sich dadurch die verstärkten Forschungsaktivitäten der USA in diesem Bereich. Ebenso könnte sich hiermit die höhere durchschnittliche Messmethodenanzahl in nordamerikanischen Studien erklären.

Ein weiterer Hinweis für den Einfluss des Gesundheitssystems auf die Art der Messmethode ist die Nichtverwendung von EDM in Ländern außerhalb von Europa und Nordamerika. Die finanziellen Mittel für die Verwendung dieser Messmethode sind in diesen Ländern meist nicht vorhanden.

Studien mit nur einem Messverfahren bieten eine veränderte Häufigkeitsverteilung der Messmethoden. Self-Report, EDM und Kollateralreport werden ca. 50-100 \% häufiger verwendet. Dabei ist gerade die Verwendung des Kollateralreports als alleinige Adhärenzmessung als äußerst kritisch zu bewerten, da er eine geringe Sensitivität hat. Die Adhärenzbeurteilung durch Ärzte ist in etwa so genau wie der Münzwurf (Stephenson et al. 1993). Self-Reports sind leicht durchzuführen und wurden deswegen wahrscheinlich häufig als alleinige Adhärenzmessmethode ausgewählt. Auch sie bieten jedoch alleine keine ausreichende Aussagekraft und sollten wenn möglich immer noch mit weiteren (objektiven) Messverfahren kombiniert werden. Das gleiche gilt für EDM, das zwar eine recht präzise Messmethode darstellt, aber eben auch Einschränkungen wie z.B. der Beeinflussung persönlicher Adhärenzstrategien, Curiosity Opening oder Pocket Dosing unterliegt.

Vor allem Studien bei psychischen Erkrankungen haben Adhärenz häufiger mit zwei oder mehr Messverfahren (Häufigkeit bei Studien mit einer Messmethode $12 \%$ vs. Häufigkeit bei Studien mit zwei oder mehr Messmethoden 22 \%) gemessen, was wie oben bereits erwähnt - auf die besonderen Herausforderungen an die Adhärenzmessung bei psychischen Erkrankungen zurückzuführen sein könnte. Studien bei mehreren Erkrankungen und vor allem bei Diabetes messen hingegen häufiger mit einer Messmethode. Gerade Studien, die mehrere Erkrankungen untersuchen, 
sind methodologisch kritisch zu betrachten, da jede Erkrankung eigene Adhärenzanforderungen besitzt. Die häufigere Verwendung nur einer Messmethode gerade bei diesen Studien ist ein weiterer Hinweis auf eine fragwürdige Methodik dieser Arbeiten.

\subsection{Zusammenfassung und Ausblick}

In unserem Review haben wir festgestellt, dass Adhärenz in den untersuchten Studien häufig unzureichend gemessen wurde. Nur ca. 5 \% der 448 untersuchten Studien erfüllten die vorher definierten Qualitätskriterien. Die wichtigste Empfehlung zur Verbesserung der Adhärenzmessung - die Verwendung mehrerer Messmethoden wurde bei den meisten Studien (336/448) nicht umgesetzt. In einer Analyse der am häufigsten verwendeten Messmethoden (Self-Report und Medication Count/Weighing) konnten wir Mängel in der Art der Durchführung feststellen. Damit ist nicht nur die Validität in Frage gestellt, auch die Interpretation und Vergleichbarkeit der Adhärenzergebnisse scheint aufgrund dieser Mängel nur eingeschränkt möglich.

Zukünftige Forschungsarbeiten sollten verwendete Begrifflichkeiten im Zusammenhang mit Adhärenz vorab definieren und dann gezielt verwenden. Auch präzisere Beschreibung von Ergebnis, Messzeitpunkt, Messhäufigkeit und Messmethode sind wichtig, um die Qualität der Studien zu verbessern.

Die einzelnen Messmethoden sowie deren Durchführung sollten nochmals gezielt untersucht werden. Ein besonderes Augenmerk sollte auf den häufig verwendeten Messmethoden Self-Report, Medication Count/Weighing und EDM liegen. Besonders der Self-Report signalisiert aufgrund seiner diversen Ausführungen und der in unserer Arbeit festgestellten Mängel weiteren Forschungsbedarf.

Das Werkzeug zur Qualitätsbewertung von Adhärenzmessung, wie wir es in dieser Arbeit eingeführt haben, bietet Raum für Weiterentwicklungen. Die Verfügbarkeit und Anwendung eines solchen Werkzeugs würde es wahrscheinlicher machen, dass sich zukünftige Untersuchungen an den hier und anderweitig gegebenen Empfehlungen orientieren. Dies könnte zu einer Qualitätssteigerung führen. 
Mehrere Autoren (Berg und Arnsten 2006, Chesney 2006) sprechen sich für eine weiterführende Diversität der Messmethoden und deren Durchführung aus, um Adhärenz besser zu verstehen. Wahrscheinlich wäre jedoch eine gewisse Standardisierung der Messmethoden und deren Durchführung sinnvoller, zumindest bei bestimmten Studien wie z.B. bei den Interventionsstudien und Studien, die Adhärenz nur sekundär bestimmen, um Vergleiche (z.B. zwischen den Interventionen) anstellen zu können. 


\section{Zusammenfassung}

Hintergrund: Adhärenz ist das Ausmaß der Einhaltung ärztlicher Behandlungsvereinbarungen und spielt gerade in der Behandlung chronischer Erkrankungen eine wichtige Rolle. Non-Adhärenz ist weit verbreitet und oft mit negativen Konsequenzen (z.B. erhöhter Mortalität und Morbidität und verminderter Lebensqualität) verbunden. Die verlässliche Messung der Adhärenz könnte dazu beitragen, die negativen Konsequenzen rechtzeitig zu erkennen bzw. zu verhindern. Trotz verschiedener Empfehlungen für eine qualitativ akzeptable Messung der Adhärenz werden entsprechende Standards offensichtlich nur ungenügend in Studien berücksichtigt.

Ziele: Mit einer systematischen Literaturübersicht wurden zwei Hauptziele verfolgt: (1) die bisherige Durchführung der Adhärenzmessung in Studien darzustellen, (2) die Qualität dieser Studien auf Basis der bisherigen Empfehlungen zur Adhärenzmessung zu bewerten. Daraus sollten sich zugleich Empfehlungen für die Adhärenzmessung in zukünftigen Studien ergeben.

Methode: Die Literaturrecherche erstreckte sich auf fünf einschlägige Datenbanken: MedLine, Embase, Cochrane Controlled Trials Register, CINAHL und PsycINFO. Es wurden alle kontrollierten pharmakologischen Studien eingeschlossen, in denen (1) Medikamentenadhärenz gemessen und (2) eine Intervention zur Adhärenzverbesserung durchgeführt wurde. Nach einer Kurz-Auswertung aller Studien, die zumindest eine Messmethode nutzten, wurden dann diejenigen Studien ausführlicher ausgewertet, die mindestens zwei unterschiedliche Messmethoden verwendet hatten („Volltext-Auswertung“). Die Qualitätskriterien dieser ausführlicheren Bewertung orientierten sich an Expertenempfehlungen zur Adhärenzmessung (Anzahl der Messmethoden, getrennte Ergebnisaufführung verschiedener Messmethoden u.a.).

Ergebnisse: Die Literaturrecherche lieferte zunächst 4.456 Studien. Nach einem kriteriengeleiteten Screening wurden 448 Studien zur weiteren Auswertung eingeschlossen. Nur 24 Studien (5\%) erfüllten die vorab definierten Qualitätskriterien vollständig bzw. fast vollständig. 336 Studien nutzten nur eine Messmethode. Die am häufigsten verwendeten Messmethoden waren hier Self-Report (45\%) und Medication Count/Weighing (17\%). In den 112 Studien mit mehr als einer Messmethode wurden im Schnitt 2,5 Messmethoden genutzt (insgesamt 278 Messungen). Auch hier waren die häufigsten Messmethoden Self-Report (32 \%) und Medication Count/Weighing (21\%). In einer Auswertung dieser Messmethoden zeigte sich, dass 
nur in $16 \%$ validierte Formen von Self-Reports genutzt wurden (14 von 88 Messungen). Häufig ließ sich z.B. gar nicht ermitteln, welche Form des Self-Reports (Interview, Fragebogen, Tagebuch etc.) verwendet wurde. Sowohl beim Self-Report als auch bei Medication Count/Weighing fehlten wichtige Informationen (z.B. ob die Messungen angekündigt oder unangekündigt durchgeführt wurden).

Schlussfolgerungen: Die für die Adhärenzmessung von Experten entwickelten Empfehlungen (z.B. Verwendung von mind. zwei Messmethoden) wurden in den untersuchten Studien größtenteils nicht umgesetzt. Auch in der Durchführung der Messmethoden bestehen große Unterschiede. Letztlich sind die Studienergebnisse dadurch inkonsistent und das Ausmaß der Adhärenz in den jeweiligen Studien lässt sich nicht valide bestimmen. Eine Standardisierung der Messdurchführung sowie eine detailierte Beschreibung der jeweiligen Adhärenzmessung bleibt weiterhin eine dringliche Forderung. 


\section{Literaturverzeichnis}

Arnsten JH, Demas PA, Farzadegan H, Grant RW, Gourevitch MN, Chang CJ, Buono D, Eckholdt H, Howard AA, Schoenbaum EE (2001): Antiretroviral therapy adherence and viral suppression in HIV-infected drug users: comparison of self-report and electronic monitoring. Clin Infect Dis $\underline{33}, 1417-1423$

Bangsberg DR, Hecht FM, Chaerlebois ED, Chesney M, Moss A (2001): Comparing objective measures of adherence to HIV antiretroviral therapy: electronic medication monitors and unannounced pill counts. AIDS Behav $\underline{5}, 275-281$

Bartlett JA (2002): Addressing the challenges of adherence. J Acquir Immune Defic Syndr 29 Suppl $1,2-10$

Berg KM, Arnsten JH (2006): Practical and conceptual challenges in measuring antiretroviral adherence. J Acquir Immune Defic Syndr 43 Suppl 1, S79-S87

Bova CA, Fennie KP, Knafl GJ, Dieckhaus KD, Watrous E, Williams AB (2005): Use of electronic monitoring devices to measure antiretroviral adherence: practical considerations. AIDS Behav $\underline{9}$, $103-110$

Cantrell CR, Eaddy MT, Shah MB, Regan TS, Sokol MC (2006): Methods for evaluating patient adherence to antidepressant therapy: a real-world comparison of adherence and economic outcomes. Med Care 44, 300-303

Chesney MA (2006): The elusive gold standard. Future perspectives for HIV adherence assessment and intervention. J Acquir Immune Defic Syndr 43 Suppl 1, 149-155

Chesney MA, Ickovics JR, Chambers DB, Gifford AL, Neidig J, Zwickl B, Wu AW (2000a): Selfreported adherence to antiretroviral medications among participants in HIV clinical trials: the AACTG adherence instruments. Patient Care Committee \& Adherence Working Group of the Outcomes Committee of the Adult AIDS Clinical Trials Group (AACTG). AIDS Care 12 (3), 255-266

Chesney MA, Morin M, Sherr L (2000b): Adherence to HIV combination therapy. Soc Sci Med $\underline{50}$, 1599-1605

Claxton AJ, Cramer J, Pierce C (2001): A systematic review of the associations between dose regimens and medication compliance. Clin Ther $\underline{23}, 1296-1310$

Colditz GA, Miller JN, Mosteller F (1989): How study design affects outcomes in comparisons of therapy. I: Medical. Stat Med $\underline{8}, 441-454$

Cramer JA, Roy A, Burrell A, Fairchild CJ, Fuldeore MJ, Ollendorf DA, Wong PK (2008): Medication Compliance and Persistence: Terminology and Definitions. Value Health $\underline{11}$, 44-47

DiMatteo MR (2004): Variations in patients' adherence to medical recommendations: a quantitative review of 50 years of research. Med Care $\underline{42}, 200-209$

DiMatteo MR, Giordani PJ, Lepper HS, Croghan TW (2002): Patient adherence and medical treatment outcomes: a meta-analysis. Med Care $\underline{40}, 794-811$

DiMatteo MR, Haskard KB (2006): Further challenges in adherence research: measurements, methodologies, and mental health care. Med Care 44, 297-299

Downs SH, Black N (1998): The feasibility of creating a checklist for the assessment of the methodological quality both of randomised and non-randomised studies of health care interventions. J Epidemiol Community Health $\underline{52}, 377-384$ 
Düsing R (2006): Therapietreue bei medikamentöser Behandlung - Adherence to medical treatment. Dtsch Med Wochenschr 131, 28-30

Farmer KC (1999): Methods for measuring and monitoring medication regimen adherence in clinical trials and clinical practice. Clin Ther $\underline{21}, 1074-1090$

Fogarty L, Roter D, Larson S, Burke J, Gillespie J, Levy R (2002): Patient adherence to HIV medication regimens: a review of published and abstract reports. Patient Educ Couns $\underline{46}, 93-108$

Freund A, Johnson SB, Silverstein J, Thomas J (1991): Assessing daily management of childhood diabetes using 24-hour recall interviews: Reliability and stability. Health Psychol 10, 200-208

Gao X, Nau DP (2000): Congruence of three self-report measures of medication adherence among HIV patients. Ann Pharmacother $\underline{34}, 1117-1122$

Gorenoi V, Schönermark MP, Hagen A: Maßnahmen zur Verbesserung der Compliance bzw. Adherence in der Arzneimitteltherapie mit Hinblick auf den Therapieerfolg; in: Schriftenreihe Health Technology Assessment, Bd. 65, 1. Auflage; hrsg. vom Deutschen Institut für Medizinische Dokumentation und Information, Köln 2007, 1-69

Gossec L, Tubach F, Dougados M, Ravaud P (2007): Reporting of adherence to medication in recent randomized controlled trials of 6 chronic diseases: a systematic literature review

1. Am J Med Sci $\underline{334}, 248-254$

Gross R, Bilker WB, Friedman HM, Coyne JC, Strom BL (2002): Provider inaccuracy in assessing adherence and outcomes with newly initiated antiretroviral therapy. AIDS $\underline{16}, 1835-1837$

Hasford J, Behrend C, Sangha O: Vergleichende Analyse und Bewertung von Methoden zur Erfassung der Compliance; in: Compliance und Selbstmanagement; hrsg. v. Petermann F; Hogrefe, Göttingen 1998, 21-44

Haynes RB (1978): Introduction; in: Compliance in Health Care; hrsg. v. Haynes RB, Sackett DL und Taylor DW; The Johns Hopkins University Press, Baltimore 1978, 1-18

Haynes RB, Gibson ES, Taylor DW, Bernholz CD, Sackett DL (1982a): Process versus outcome in hypertension: a positive result. Circulation $\underline{65}, 28-33$

Haynes RB, Taylor D.W., Sackett D.L.: Compliance Handbuch; R.Oldenbourg Verlag, München $1982 \mathrm{~b}$

Haynes RB, Yao X, Degani A, Kripalani S, Garg A, McDonald HP (2005): Interventions to enhance medication adherence. Cochrane Database Syst Rev, Art. No.: CD000011

Heier M (2006): Mein Patient macht nicht, was ich will. Frankfurter Allgemeine Sonntagszeitung v. 11.06.2006, 71

Heuer HO, Heuer S, Lennecke K (1999): Compliance in der Arzneimitteltherapie - Von der NonCompliance zur pharmazeutischen Kooperation; Wissenschaftliche Verlagsgesellschaft GmbH, Stuttgart 1999

Higgins JPG, Green S, editors (2006): Cochrane Handbook for Systematic Reviews of Interventions 4.2.6 [updated September 2006]. The Cochrane Library, $<$ http://www.cochrane.org/resources/handbook/Handbook4.2.6Sep2006.pdf last accessed 29.06.2009)>

Irvine J, Baker B, Smith J, Jandciu S, Paquette M, Cairns J, Connolly S, Roberts R, Gent M, Dorian $P$ (1999): Poor adherence to placebo or amiodarone therapy predicts mortality: results from the CAMIAT study. Canadian Amiodarone Myocardial Infarction Arrhythmia Trial. Psychosom Med $\underline{61}, 566-575$ 
Johnson AM, Copas AJ, Erens B, Mandalia S, Fenton K, Korovessis C, Wellings K, Field J (2001): Effect of computer-assisted self-interviews on reporting of sexual HIV risk behaviours in a general population sample: a methodological experiment. AIDS $\underline{15}, 111-115$

Johnson SB, Silverstein J, Rosenbloom A, Carter R, Cunningham W (1986): Assessing daily management in childhood diabetes. Health Psychol $\underline{5}, 545-564$

Kripalani S, Yao X, Haynes RB (2007): Interventions to enhance medication adherence in chronic medical conditions: a systematic review. Arch Intern Med 167, 540-550

Kyngas H, Duffy ME, Kroll T (2000): Conceptual analysis of compliance. J Clin Nurs $\underline{9}$, 5-12

Lewis A (1997): Noncompliance: a $\$ 100$ billion problem. Remington Report $\underline{5}$, 14-15

Liu H, Golin CE, Miller LG, Hays RD, Beck CK, Sanandaji S, Christian J, Maldonado T, Duran D, Kaplan AH, Wenger NS (2001): A comparison study of multiple measures of adherence to HIV protease inhibitors. Ann Intern Med 134, 968-977

Macalino GE, Celentano DD, Latkin C, Strathdee SA, Vlahov D (2002): Risk behaviors by audio computer-assisted self-interviews among HIV-seropositive and HIV-seronegative injection drug users. AIDS Educ Prev 14, 367-378

MacLaughlin EJ, Raehl CL, Treadway AK, Sterling TL, Zoller DP, Bond CA (2005): Assessing medication adherence in the elderly: which tools to use in clinical practice? Drugs Aging 22, 231255

Mannheimer S., Thackeray L., Huppler Hullsiek K., Chesney M., Gardner E., Wu A., Telzak E., Lawrence J., Baxter J., Friedland G. (2006): A randomized comparison of two instruments for assessing self-reported antiretroviral adherence. J Int Assoc Physicians AIDS Care $\underline{5}, 57-82$

Markowitz M (1970): Eradication of rheumatic fever: an unfulfilled hope. Circulation 411, 1077-1084

Metzger DS, Koblin B, Turner C, Navaline H, Valenti F, Holte S, Gross M, Sheon A, Miller H, Cooley P, Seage GR (2000): Randomized controlled trial of audio computer-assisted selfinterviewing: utility and acceptability in longitudinal studies. HIVNET Vaccine Preparedness Study Protocol Team. Am J Epidemiol 152, 99-106

Morisky, E., Green L, Levine, .M. (1986): Concurrent and predictive validity of a self-reported measure of medication adherence. Med Care 24 (1), 67-74

Moss AR, Hahn JA, Perry S, Charlebois ED, Guzman D, Clark RA, Bangsberg DR (2004): Adherence to highly active antiretroviral therapy in the homeless population in San Francisco: a prospective study. Clin Infect Dis $\underline{39}$, 1190-1198

Murri R, Antinori A, Ammassari A, Nappa S, Orofino G, Abrescia N, Mussini C, D'Arminio MA, Wu AW (2002): Physician estimates of adherence and the patient-physician relationship as a setting to improve adherence to antiretroviral therapy. J Acquir Immune Defic Syndr 31 Suppl 3 , S158-S162

Nichol MB, Venturini F, Sung JC (1999): A critical evaluation of the methodology of the literature on medication compliance. Ann Pharmacother $\underline{33}, 531-540$

OECD (2008): OECD Health Data 2008. OECD < http://www.ecosante.org/oecd.htm - last accessed 25.01.2009>

Okuno J, Tomura S, Yanagi H (2002): Treated Hypertensives with Good Medication Compliance are Still in a State of Uncontrolled Blood Pressure in the Japanese Elderly. Environ Health Prev Med 7, 193-198 
Paterson C, Britten N (2005): A narrative review shows the unvalidated use of self-report questionnaires for individual medication as outcome measures. J Clin Epidemiol $\underline{58}, 967-973$

Peterson AM, Nau DP, Cramer JA, Benner J, Gwadry-Sridhar F, Nichol M (2007): A checklist for medication compliance and persistence studies using retrospective databases. Value Health $\underline{10}$, 3-12

Phillips B, Ball C, Sackett D, Badenoch D, Straus D, Haynes B, Dawes M (2001): Levels of Evidence and Grades of Recommendation. Oxford: Oxford-Centre for Evidence Based Medicine $2001<$ http://www.cebm.net/levels of evidence.asp last accessed December 2008>

Pullar T, Kumar S, Tindall H, Feely M (1989): Time to stop counting the tablets? Clin Pharmacol Ther $\underline{46}, 163-168$

Rasmussen JN, Chong A, Alter DA (10-1-2007): Relationship between adherence to evidencebased pharmacotherapy and long-term mortality after acute myocardial infarction. JAMA 297, $177-186$

Roter DL, Hall JA, Merisca R, Nordstrom B, Cretin D, Svarstad B (1998): Effectiveness of interventions to improve patient compliance: a meta-analysis. Med Care $\underline{36}, 1138-1161$

Rudd P, Byyny RL, Zachary V, LoVerde ME, Titus C, Mitchell WD, Marshall G (1989): The natural history of medication compliance in a drug trial: limitations of pill counts. Clin Pharmacol Ther $\underline{46}$, 169-176

Sabate E (2003): Adherence to long-term therapies: evidence for action. Geneva: World Health Organisation <http://www. who.int/medicinedocs/collect/medicinedocs/pdf/s4883e/s4883e.pdf last accessed December 2008>

Sackett DL: Introduction and the magnitude of compliance and non-compliance; in: Compliance with Therapeutic Regimens; hrsg. v. Sackett DL und Haynes RB; The Johns Hopkins University Press, Baltimore 1976, 1-25

Samet JH, Sullivan LM, Traphagen ET, Ickovics JR (2001): Measuring adherence among HIVinfected persons: is MEMS consummate technology? AIDS Behav $\underline{5}, 21-30$

Schilling LS, Grey M, Knafl KA (2002): A review of selfmanagement in type-1 diabetes by youth and their parents. The Diabetes Educator $\underline{28}, 796-808$

Simoni JM, Kurth AE, Pearson CR, Pantalone DW, Merrill JO, Frick PA (2006): Self-Report Measures of Antiretroviral Therapy Adherence: A Review with Recommendations for HIV Research and Clinical Management. AIDS Behav 10, 227-245

Simpson SH, Eurich DT, Majumdar SR, Padwal RS, Tsuyuki RT, Varney J, Johnson JA (2006): A meta-analysis of the association between adherence to drug therapy and mortality. BMJ $\underline{333}, 15$

Stephenson BJ, Rowe BH, Haynes RB, Macharia WM, Leon G (1993): The rational clinical examination. Is this patient taking the treatment as prescribed? JAMA $\underline{269}, 2779-2781$

Svarstad, .L., Chewning BA, Sleath BL, Claesson C (1999): The Brief Medication Questionnaire: A tool for screening patient adherence and barriers to adherence. Patient Educ Counsel $\underline{37}, 113-$ 124

Takiya LN, Peterson AM, Finley RS (2004): Meta-analysis of interventions for medication adherence to antihypertensives. Ann Pharmacother $\underline{38}, 1617-1624$

Vermeire E, Hearnshaw H, Van Royen P, Denekens J (2001): Patient adherence to treatment: three decades of research. J Clin Pharm Ther $\underline{26}, 331-342$ 
Vincke J, Bolton R (2002): Therapy adherence and highly active antiretroviral therapy: comparison of three sources of information. AIDS Patient Care STDS $\underline{16}$, 487-495

Walsh JC, Mandalia S, Gazzard BG (2002): Responses to a 1 month self-report on adherence to antiretroviral therapy are consistent with electronic data and virological treatment outcome. AIDS 16, $269-277$

Wendel CS, Mohler MJ, Kroesen K, Ampel NM, Gifford AL, Coons SJ (2001): Barriers to use of electronic adherence monitoring in an HIV clinic. Ann Pharmacother $\underline{35}$, 1010-1015

WHO (2000): The World Health Report 2000 - Health systems: improving performance. Geneva: WHO <http://www.who.int/entity/whr/2000/en/whr00 en.pdf - last accessed 25.01.2009>

Wiener L, Riekert K, Ryder C, Wood LV (2004): Assessing medication adherence in adolescents with HIV when electronic monitoring is not feasible. AIDS Patient Care STDS $\underline{18}, 527-538$

Wilker FW: Compliance; in: Medizinische Psychologie und Soziologie; hrsg. v. Wilker FW, Bischoff C und Novak P; Urban \& Schwarzenberg, München 1994, 284-291

Willich S (2006): Randomisierte kontrollierte Studien: Pragmatische Ansätze erforderlich. Dtsch Arztebl $\underline{103(39)}, 2524-2529$ 


\section{Danksagung}

Für die freundliche Überlassung des Themas der Dissertation danke ich Herrn Prof. Dr. med. MM Kochen, MPH, FRCGP und Herrn Prof. Dr. disc. pol. W. Himmel.

Mein ganz besonderer Dank gilt Prof. Dr. disc. pol. W. Himmel und Prof. Dr. med. Martin Scherer für die nette Betreuung und die vielen wichtigen Anregungen. Weiterhin danke ich den Mitarbeitern der Abteilung Allgemeinmedizin der Universität Göttingen für die freundliche Atmosphäre und stets kollegiale Unterstützung.

Meinen lieben Eltern, Geschwistern und Thomas Held danke ich für tatkräftigen Beistand und Geduld. 


\section{Lebenslauf}

Ich wurde am 19.02.1981 als Sohn von Frau Gabriele Schönborn und Herr Priv.-Doz. Dr. med. Jürgen Schönborn in Olpe geboren. Mein Abitur erlangte ich an der Landgraf-Ludwig-Schule in Gießen im Jahr 2000. Im direkten Anschluss leistete ich meinen Grundwehrdienst ab.

Zum Wintersemester 2001/2002 begann ich das Studium der Biologie an der Technischen Universität Darmstadt, das ich aber nach einem Semester abbrach. Im April 2002 nahm ich das Studium der Humanmedizin an der Georg-August-Universität in Göttingen auf und konnte im April 2004 den vorklinischen Studienabschnitt abschlieBen. Nach einem Auslandsaufenthalt in Trondheim (Norwegen) im Sommer 2007 absolvierte ich mein praktisches Jahr in den Fächern Chirurgie (Royal Hobart Hospital, Australien), Hals-Nasen-Ohren-Heilkunde (Universitätsklinikum Göttingen) und Innere Medizin (Evang. Krankenhaus Weende). Im Juni 2009 konnte ich die zweite Ärztliche Prüfung mit der Gesamtnote 2 beenden.

In der Abteilung Allgemeinmedizin der Georg-August-Universität Göttingen begann ich 2006 meine Dissertationsarbeiten. Begleitend war ich von 2006 bis 2007 in der Abteilung Allgemeinmedizin als studentische Hilfskraft im Bereich Lehre und Forschungsarbeiten tätig. 